\title{
Involvement of Reversible Protein Methylation in 13-Lined Ground Squirrel Hibernation.
}

By
Alexander Watts
BSc. (Hons.), 2014
Carleton University

A thesis submitted to the Faculty of Graduate and Postdoctoral Affairs in partial fulfillment of the requirements for the degree of

\author{
Master of Science \\ Department of Biology \\ Carleton University \\ Ottawa, Ontario, Canada
}

C) Copyright 2016

Alexander Watts 
The undersigned hereby recommend to the Faculty of Graduate Studies and Research acceptance of this thesis:

\title{
The Role of Reversible Protein Methylation in Regulating Mammalian Hibernation in Ictidomys tridecemlineatus.
}

\author{
Submitted by: \\ Alexander Watts \\ in partial fulfillment of the requirements for the degree of Master of Science
}

Chair, Department of Biology

Thesis Supervisor

Carleton University 


\begin{abstract}
During winter hibernation, body temperature falls to near ambient levels, metabolism is shifted to favor lipid oxidation and transcriptional and translational activity is minimized in the face of limited resources and increased heat generation costs. In order to regulate such profound changes, mammals require control at least partly brought about by protein post-translational modifications. Protein lysine methylation provides a mechanism by which enzymes may alter the activity, stability and modification states of proteins relevant to hibernator physiology. Protein abundance of SMYD2, SUV39H1, SET8, SET7/9, G9a, ASH2L and RBBP5 in 13-lined ground squirrel (Ictidomys tridecemlineatus) skeletal muscle and liver was characterized. Tissue-specific regulation was seen and enzymes changed during either torpor, arousal, or transitory periods. Methylation of $\mathrm{H} 3$, HSP90, and p53 proteins were also quantified and typically followed patterns of modifying enzymes. Overall, these experiments show protein lysine methylation is differentially regulated during 13-lined ground squirrel hibernation.
\end{abstract}




\section{Acknowledgements}

I wish to dearly thank all the members and students involved in the work that takes place within the Storey-lab, especially Ken, Jan, the fellow graduate students who started our journey together, and absolutely everyone who makes sure that ground-breaking research is consistently produced here.

Secondly, my friends and family were instrumental in helping me stay focused, determined and dedicated to my thesis work.

I am sure that without the people mentioned above, the work, writing and effort placed into this research would be severely lacking compared to its current state.

Finally, I wish to thank Bernie Sanders for keeping my hope for political sensibility alive, and then the American people for quickly squashing those dreams and reminding me of reality. Penn and Teller are also great! 


\section{Table of Contents}

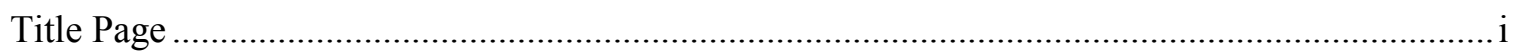

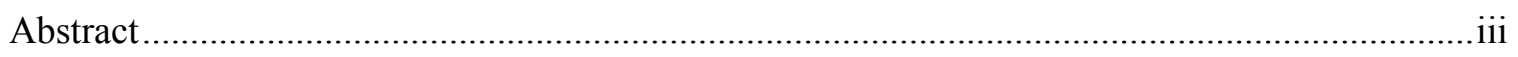

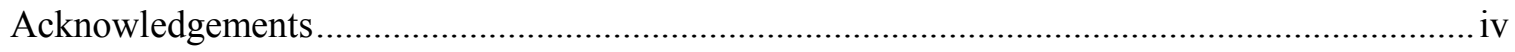

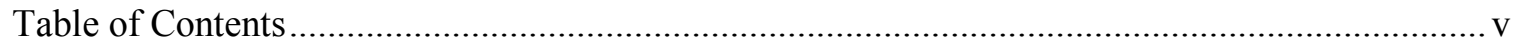

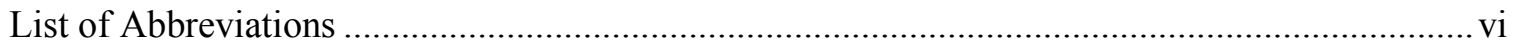

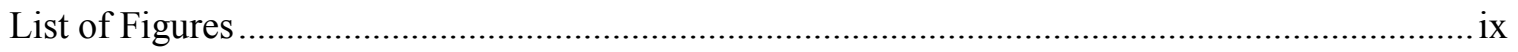

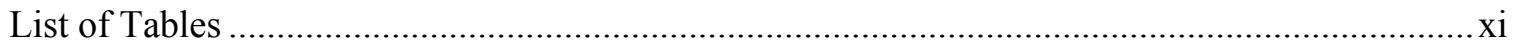

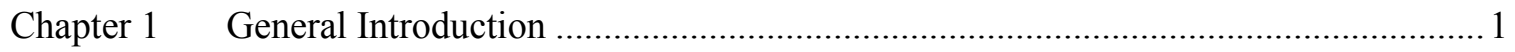

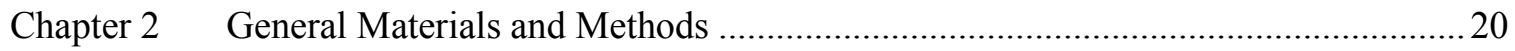

Chapter 3 Analysis of Histone Methyltransferase Enzyme Abundance and their Downstream

Targets in Skeletal Muscle during Mammalian Hibernation .........................................28

Chapter 4 Analysis of Histone Methyltransferase Enzyme Abundance and their Downstream

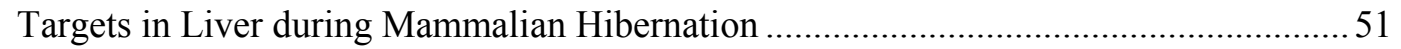

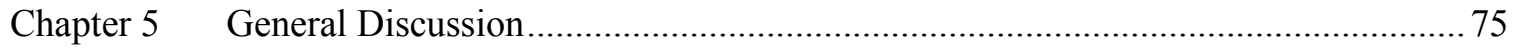

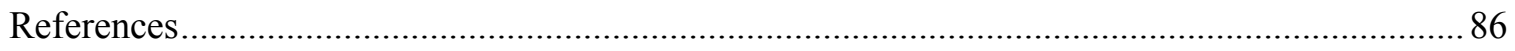

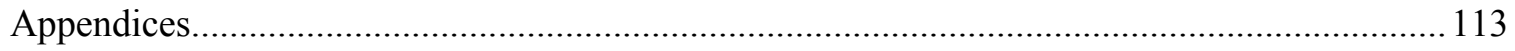




\section{List of Abbreviations}

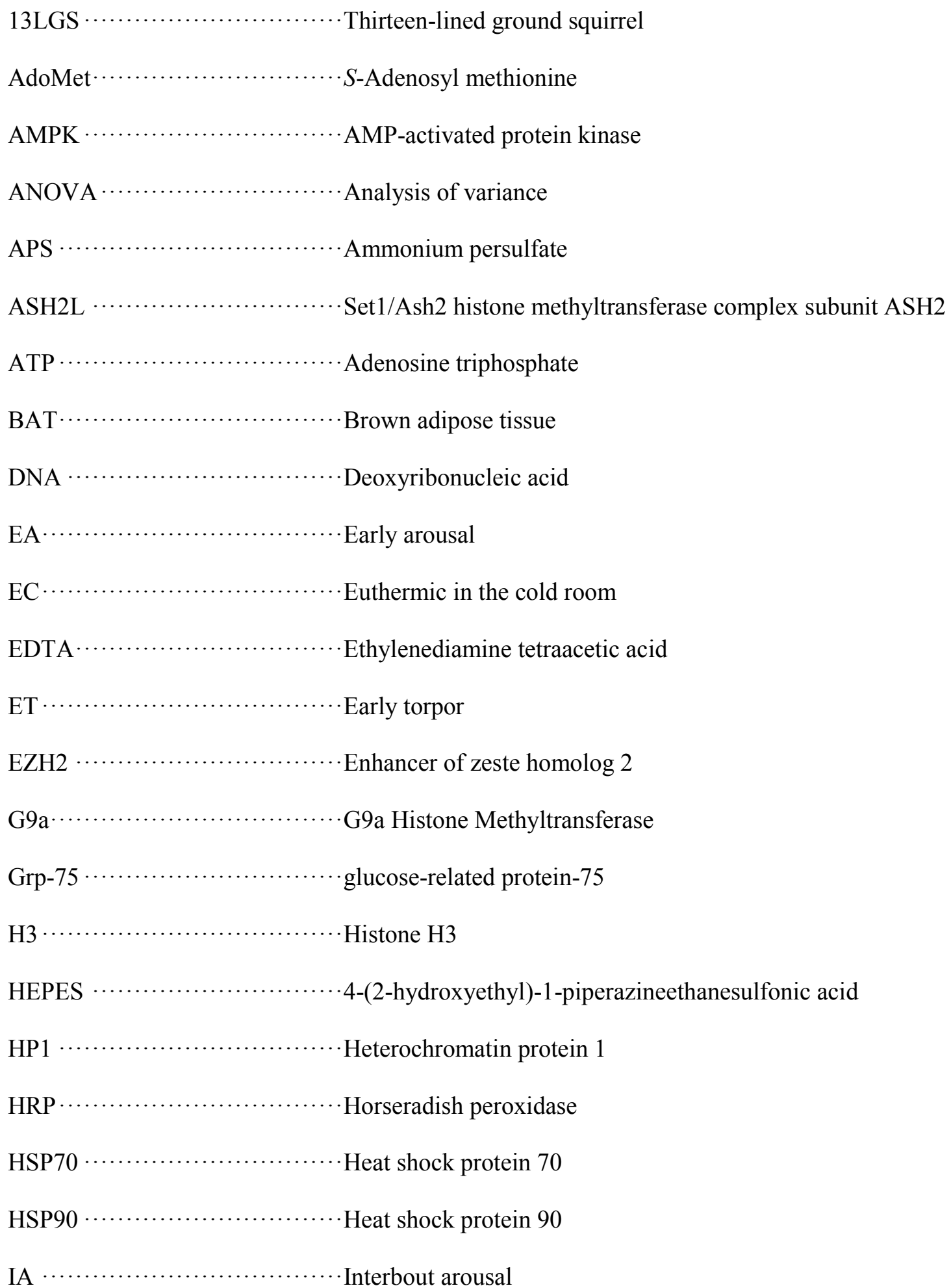




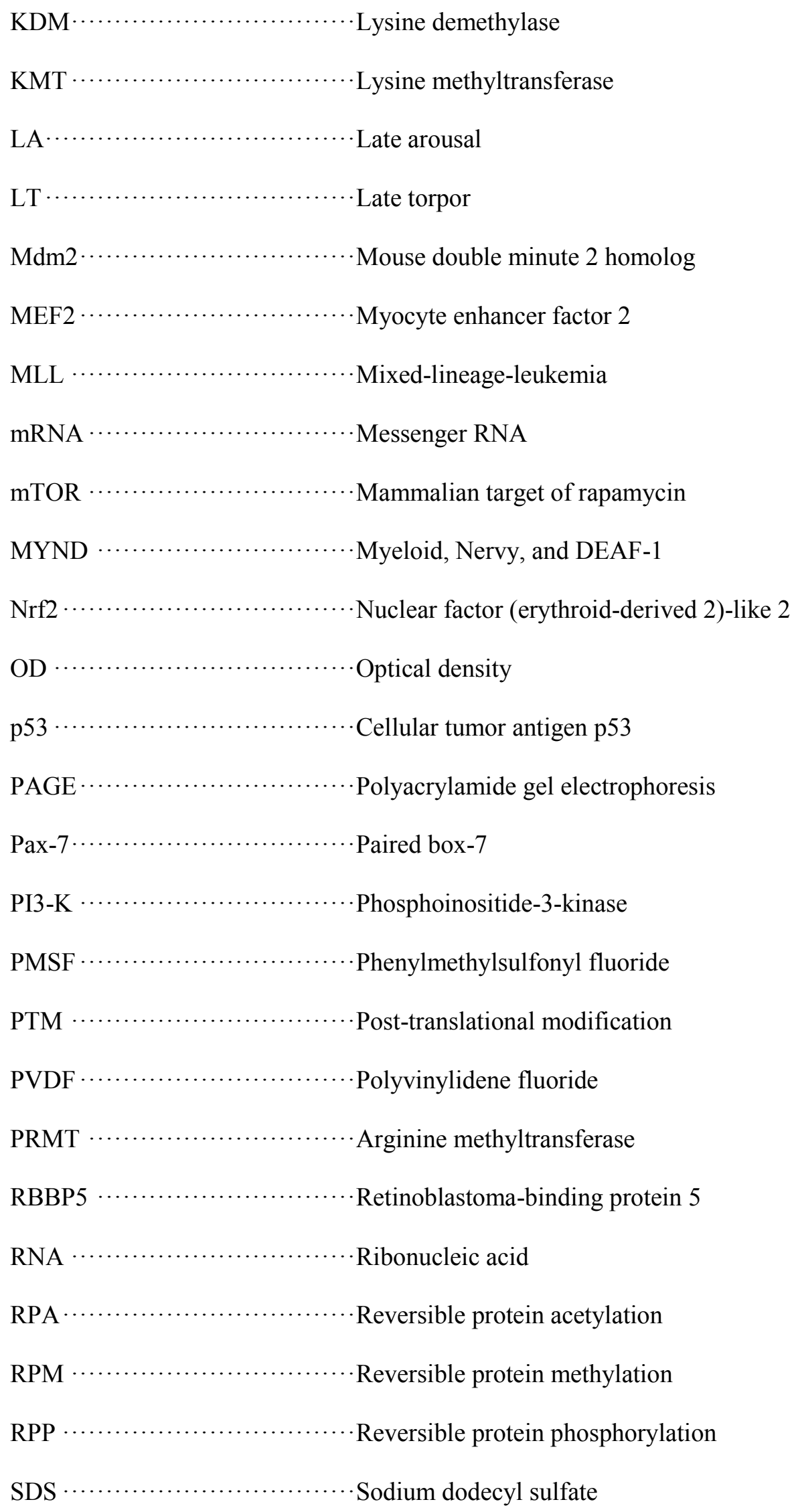




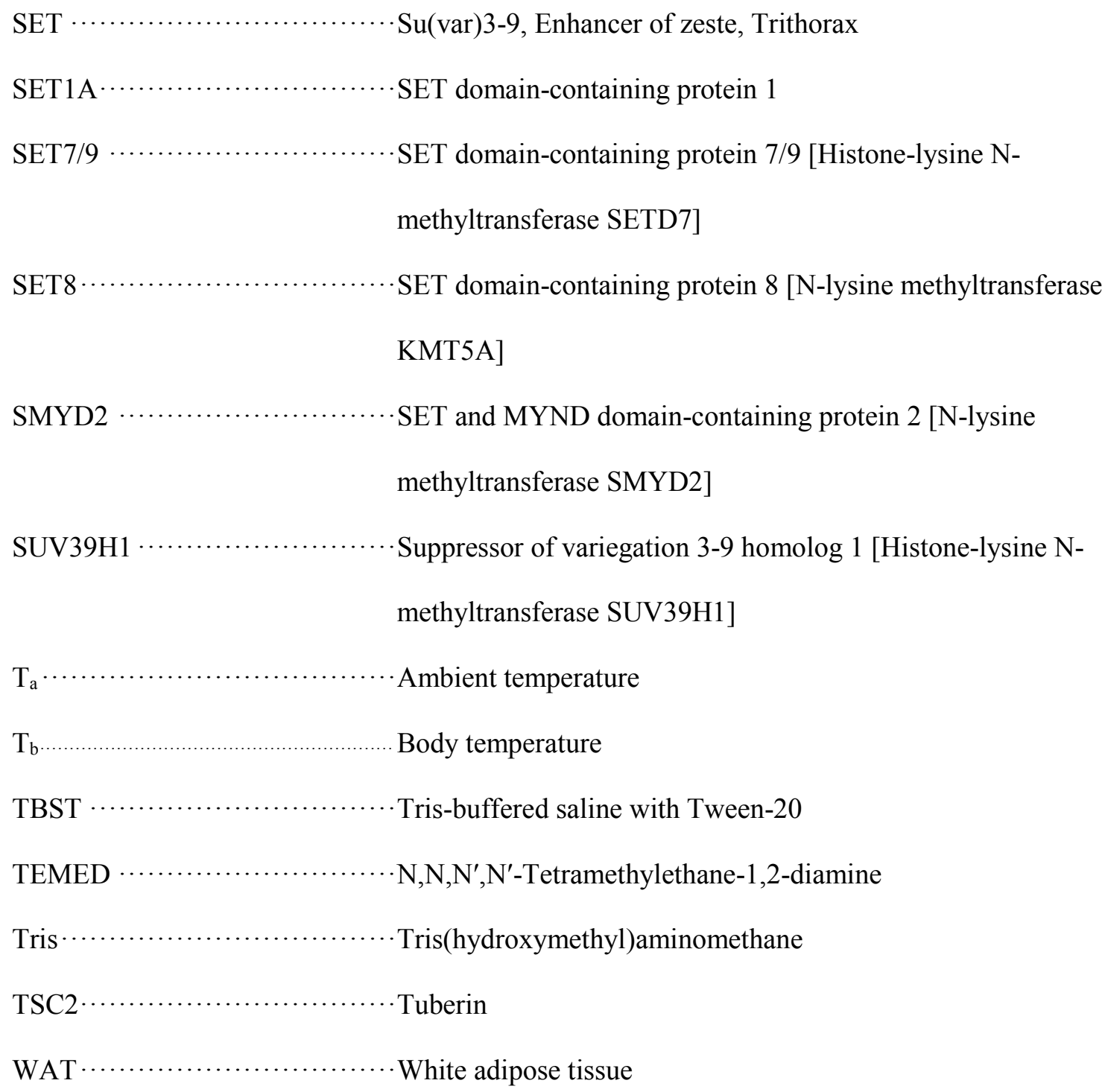




\section{List of Figures}

Figure 1.1: A representation of torpor/arousal cycles during hibernation 18

Figure 3.1: Relative protein abundance of SUV39H1, SMYD2, SET8 and SET7/9 in skeletal muscle of I. tridecemlineatus over the torpor/arousal cycle along with three representative immunoblot protein bands for EC and LT sampling points 46

Figure 3.2: Relative protein abundance of G9a, ASH2L and RBBP5 in skeletal muscle of $I$. tridecemlineatus over the torpor/arousal cycle along with three representative immunoblot protein bands for EC and LT sampling points

Figure 3.3: Relative histone methyltransferase activity at $\mathrm{H} 3 \mathrm{~K} 4, \mathrm{H} 3 \mathrm{~K} 9$ and $\mathrm{H} 3 \mathrm{~K} 27$ in skeletal muscle total soluble protein extracts from I. tridecmlineatus .48

Figure 3.4: Relative amounts of histone $\mathrm{H} 3$ monomethylated at lysine 4 (H3K4me), trimethylated at K9 (H3K9me $\left.{ }^{3}\right)$, monomethylated at K27 (H3K27me), or dimethylated at K36 $\left(\mathrm{H} 3 \mathrm{~K} 36 \mathrm{me}^{2}\right)$ in skeletal muscle of I. tridecemlineatus over the torpor/arousal cycle along with three representative immunoblot protein bands for EC and LT sampling points 49

Figure 3.5: Relative protein abundance of HSP90 and methyllysine (quantified at 90kDa) in skeletal muscle of I. tridecemlineatus over the torpor/arousal cycle along with three representative immunoblot protein bands for EC and LT sampling points

Figure 4.1: Relative protein abundance of SMYD2, SET8 SET7/9 and RBBP5 in liver of $I$. tridecemlineatus over the torpor/arousal cycle along with three representative immunoblot protein bands for EC and LT sampling points

Figure 4.2: Relative protein abundance of ASH2L and RBBP5 in liver of I. tridecemlineatus over the torpor/arousal cycle along with three representative immunoblot protein bands for EC and LT sampling points. 
Figure 4.3: Relative histone methyltransferase activity at H3K4, H3K9 and H3K27 in skeletal muscle total soluble protein extracts from I. tridecmlineatus

Figure 4.4: Relative amounts of histone $\mathrm{H} 3$ that is monomethylated at $\mathrm{K} 4$ (H3K4me), timethylated at $\mathrm{K} 9\left(\mathrm{H} 3 \mathrm{~K} 9 \mathrm{me}^{3}\right)$ or dimethylated at $\mathrm{K} 36\left(\mathrm{H} 3 \mathrm{~K} 36 \mathrm{me}^{2}\right)$ in liver of $I$. tridecemlineatus over the torpor/arousal cycle along with three representative immunoblot protein bands for EC and EN sampling points .73

Figure 4.5: Relative amounts of p53 that is dimethylated at $\mathrm{K} 370\left(\mathrm{p} 53 \mathrm{~K} 370 \mathrm{me}^{2}\right)$ or monomethylated at K370 (p53K370me) in liver of I. tridecemlineatus over the torpor/arousal cycle along with three representative immunoblot protein bands for EC and LT sampling points .74 


\section{List of Tables}

Table 1.1: Family and substrate specificity for KMT enzymes of relevance to this thesis 


\section{Chapter 1}

\section{General Introduction}




\section{Hypometabolic States as Overwintering Adaptations}

One of the most interesting adaptations that many species possess is the ability to survive extended periods of extreme environmental stress by entering a hypometabolic, or dormant, state in which cellular and metabolic pathways are drastically altered. One such environmental change is the seasonal transition to winter which forces various animal species to survive bouts of decreased temperature, oxygen availability, and resource supply (Storey, 1990; Storey and Storey, 2007). Overwintering adaptations can appear in a variety of organisms and take on many different strategies to combat changes from the summer environment.

Many amphibians or reptiles that are found at high latitudes descend either below the ground or below water to avoid prolonged exposure to temperatures below $0^{\circ} \mathrm{C}$; for example, the freeze-tolerant wood frog Rana sylvatica, spends winter on the forest floor where it can endure the freezing of about $65 \%$ of its total body water as extracellular ice. On the other hand, the redeared slider turtle Trachemys scripta elegans, actually avoids freezing by enduring several months of oxygen deprivation, unfrozen at the bottom of ice-covered ponds or streams (Krivoruchko and Storey, 2015; Storey and Storey, 2013). Hypometabolic strategies of overwintering are not restricted to non-mammalian phylogenies; yet another form of hypometabolism can be observed in small mammalian species.

\section{Mammalian Hibernation}

Hibernating mammalian species from such diverse groups as Macroscelidea (elephant shrews), Rodentia (ground squirrels, prairie dogs, chipmunks, dormice), Primates (lemurs), Chiroptera (bats), and Eulipotyphia (hedgehogs) shield themselves from deep cold in a burrow, tree hole, cave or other hibernaculum, where they enter hibernation that is characterized by sustained (days to weeks) metabolic rate depression (torpor) broken up by short (hours to a day) euthermic arousal periods (Carey et al., 2003; Humphries et al., 2003). A distinction should be drawn between mammals that enter short periods of dormancy in the face of cold-stress or food restriction (facultative hibernators) and mammals which rely on seasonal torpor/arousal cycles to 
survive winter (obligate hibernators), however. By not only depressing their vital processes once, but numerous times each winter, and each time ceasing processes that are vital for homeostasis in many endotherms, small mammalian obligate hibernators display one of the most remarkable and complex hypometabolic strategies for overwintering (Carey et al., 2003; Wu and Storey, 2016).

Simply put, small mammalian hibernators, including the thirteen lined ground squirrel (13LGS), Ictidomys tridecemlineatus, require a great deal of phenotypic and metabolic plasticity in order to accomplish such a feat (Pałyga, 2012; Storey, 1997; Storey and Storey, 2010).

Phenotypic Changes Associated with 13LGS Hibernation. 13LGSs are obligate hibernators, meaning they will hibernate from autumn and through winter, regardless of location, or ambient temperatures $\left(T_{a}\right)$. Since 13LGSs are not triggered to enter torpor by $T_{a}$ changes, and since decreases in body temperature $\left(\mathrm{T}_{\mathrm{b}}\right)$ and metabolic rate lag behind $\mathrm{T}_{\mathrm{a}}$ changes, it is most likely that changes in 13LGS physiology during the fall are essential to allow 13LGSs to enter torpid states (Kisser and Goodwin, 2012; Russell et al., 2010). In fact, it would appear that without the animals' seasonal changes in physiology before hibernation has even begun, descent into the torpor/arousal cycle would not be possible (Hampton and Andrews, 2007; Hampton et al., 2011).

Seasonal adjustments required to enter hibernation. Before a 13LGS may descend into a hypometabolic state, the animal needs to go through a period of hyperphagia, requiring a massive shift in dietary resources, and altering metabolite concentrations and cellular activities in a variety of tissues (Humphries et al., 2003; Storey, 2010). Sufficient body fuel reserves are required to allow the animal to survive the winter season without eating - essentially in a state of long term fasting - and a pre-hibernation fattening accounts for an about $60 \%$ increase in body weight gained compared to active animals sampled in the summer, largely due to increasing lipid reserves within the animal's adipose stores. Pre-hibernation mass gain is accomplished through increased food consumption during late summer and fall seasons, and is linked to increased 
circulating insulin concentrations (Boyer et al., 1993; Boyer and Barnes, 1999; Florant et al., 1990).

Levels of circulating insulin, released by the pancreas, are elevated in the pre-hibernation period. The hormone insulin binds membrane receptors initiating a signaling cascade which ultimately promotes glycogen's synthesis and deposition within the liver and muscle, as well as lipid synthesis and triglyceride production. During entry to torpid states, insulin serves to increase the activity of lipogenic enzymes and proteins that deposit fat into white adipose tissue (WAT) stores and build up metabolic fuel stores (Mostafa et al., 1993; Otis et al., 2011; Wang et al., 1997; Woods and Porte, 1978). For example, protein levels and enzyme activities of lipoprotein lipase, fatty acid synthase, and diacylglycerol acetyltransferase are all elevated during ground squirrel seasonal mass gain (Mostafa et al., 1993; Wang et al., 1997). In response to increased adipose stores and increased WAT cell size (Dark, 2005; Otis et al., 2011), digestive satiety signals (i.e., leptin) are released by adipocytes. While leptin typically has the effect of suppressing appetite and enhancing lipid oxidation, interestingly, its anorexigenic effects are not experienced by hibernators during the seasonal pre-hibernation mass gain (Florant and Healy, 2012; Healy and Florant, 2012). Therefore, while levels of serum insulin increase during the fall, typical of any hyperphagic state, a resistance to satiety signals is also experienced in hibernators that ultimately allows for increases in WAT mass that give the 13LGS adequate fuel supplies through lipid catabolism to last through the hibernation fast (Schwartz et al., 2015; Wu et al., 2013).

Another hormone which plays an important role in the pre-hibernation mass gain is ghrelin. The orexigenic hormone ghrelin increases feeding signals following release from the gut, stimulating appetite and carbohydrate catabolism and inhibiting lipogenesis during times of fasting (Pinkney, 2014; Sangiao-Alvarellos et al., 2009; Toshinai et al., 2001). Although ghrelin levels typically decrease during periods of increased adiposity, levels of ghrelin are actually increased in ground squirrels during the fall seasonal mass gain compared to summer and 
hibernating animals (Healy et al., 2010). Additionally, ghrelin typically coordinates the activation of AMP-activated protein kinase (AMPK) via reversible protein phosphorylation (RPP), inhibiting both fatty acid production and glycogen synthesis (Florant et al., 2010; Hardie et al., 2006; Healy et al., 2011a). This expected increase in RPP is not seen during seasonal mass gain in ground squirrel adipose (Healy et al., 2011b) and it is therefore likely that ghrelin is regulated on a seasonal basis, whereby dissociation from AMPK regulation allows for increases in WAT mass and food intake beyond that which is typically experienced during summer (Florant and Healy, 2012; Klug and Brigham, 2015).

Entry into torpor and the torpid state. During torpor in the 13LGS, characteristics of mammalian life are significantly decreased for days at a time - e.g., for animals hibernating at a $\mathrm{T}_{\mathrm{b}}$ near $0^{\circ} \mathrm{C}$, breathing rates are reduced from $100-200$ breaths/min to $4-6$ breaths/min, and heart rates drop from 200-300 beats/min to 3-5 beats/min (Boyer and Barnes, 1999; Nedergaard et al., 1990; Refinetti, 1996; Storey, 2010). These physiological changes enable the animal's metabolic rate to plummet often to just 2-4\% of resting summer values (Carey et al., 2003).

During the shift to a hypometabolic state, the majority of carbohydrate metabolism is suspended during hibernation and instead lipolytic enzyme activity is upregulated in order to ensure that a majority of the hibernator's energy demands are met by lipid oxidation derived from built-up adipose stores (Hittel and Storey, 2001; Storey and Storey, 2010). Similarly, levels of pancreatic triacylglycerol lipase, which stimulates lipolysis via the breakdown of circulating adiposomes, are upregulated, as are levels of fatty acid binding protein, fatty acid transporter, and enzymes involved in ketone production such as hydroxymethylglutaryl-CoA synthase, all of which support the switch to increased reliance on the beta-oxidation pathway (Epperson et al., 2010a). Levels of pyruvate dehydrogenase kinase isozyme 4, which inhibits glycolysis through RPP of pyruvate dehydrogenase, are also upregulated within muscle, heart, liver and WAT tissues (Andrews et al., 1998). 
Due to increases in fatty acid catabolism, and concurrent decreases in glycolysis, blood glucose is at an annual minimum during hibernation (Buck and Barnes, 1999), in turn causing reductions in insulin levels (Bauman et al., 1987; Woods and Porte, 1978). Interestingly, increased levels of glucagon, which would be expected to occur in the face of lowered circulating glucose levels, are not seen during mammalian hibernation (Bauman et al., 1987; Hoo-Paris et al., 1985). However, a shift in the plasma glucagon to insulin ratio does occur which favor the effects of glucagon and in turn, poises catabolism towards the breakdown of glycogen and the activation of WAT lipolytic enzymes (i.e., hormone sensitive lipase), as well as decreased glycolytic activity via degradation and inhibition of pyruvate kinase (Dark, 2005; Wilson et al., 1992). In fact, the shift towards fatty acid catabolism is so extensive that even the breakdown of glycogen is inhibited following decreases in activating-RPP on liver stores of glycogen phosphorylase (Storey, 1987; 1997). Clearly then, multiple systems ensure that during torpor in the 13LGS, glycolysis is suppressed while metabolic fuel requirements are fulfilled almost entirely via lipid oxidation.

Decreased insulin levels further inhibit glucose uptake by the peripheral tissues via decreased stimulation of glucose transporter type 4 (Satoh, 2014; Tessier and Storey, 2010; Wu et al., 2013). Glucose uptake is further inhibited by decreased phosphoinositide-3-kinase (PI3-K) mediated activation of the serine/threonine kinase Akt, resulting from decreased insulin binding to membrane receptors, which causes a significant regulatory shift within the liver and skeletal muscle tissues (Abnous et al., 2008; 2010). Furthermore, levels of downstream targets of Akt, including mammalian target of rapamycin (mTOR) and tuberin (TSC2), are not changed during torpor but their activated forms were significantly suppressed during torpor - lowering rates of gene transcription and protein synthesis (Wu and Storey, 2012a).

Decreased rates of gene transcription and protein translation are common findings in studies on a variety of hibernating mammals (Morin and Storey, 2009; Storey, 2003; Storey et al., 2010), including 13LGS (Frerichs et al., 1998; Morin and Storey, 2006; Tessier and Storey, 
2014). Protein synthesis is a major consumer of a euthermic mammals' energy expenditure, and its downregulation during hibernation makes sense in order to conserve fuel reserves for only the most essential cellular tasks (Heldmaier et al., 2004), and this has been shown to be the case in a variety of 13LGS tissues including brain, liver, kidney, brown adipose tissue (BAT) and digestive organs (Biggar and Storey, 2014; Hittel and Storey, 2002). Decreases in gene expression and protein synthesis are however, not global over the entire genome but rather, are specific to genes that are not essential to the hibernator's switch between euthermia and torpor or the maintenance of either physiological state (Epperson et al., 2010a; 2010b; O'Hara et al., 1999). As an example within the heart of hibernating 13LGS, the transcription factor myocyte enhancer factor 2 (MEF2) is upregulated and activated through RPP leading to increased levels of the cardioprotective proteins desmin and myomesin (Tessier and Storey, 2012). Similarly, changes in RPP of ribosomal initiation and elongation factors (Frerichs et al., 1998; van Breukelen and Martin, 2001), as well as proteins that lower messenger RNA (mRNA) turnover (i.e., poly(A) binding protein) and assist in stabilizing and folding existing proteins (i.e., heat-shock proteins) contribute to decreasing cellular energy usage during hibernation (Fahlman et al., 2000; Knight et al., 2000; $\mathrm{Wu}$ et al., 2015). Given that regulation over genome suppression is seen in combination with upregulation of certain genes whose products function in either cellular metabolism or preservation, it is clear that an intricate level of selection over biological pathways is required.

Arousal from torpor and interbout arousal. Intermittent arousals from torpor are commonplace over the hibernation season (Fons et al., 1997; Lovegrove et al., 1999; Mzilikazi et al., 2002; Wang, 1979) and, as such, mammals must have a way to reversibly return their bodies to euthermic $T_{b}$ values and facilitate the necessary increases in cellular metabolism that will increase $T_{b}$ above $T_{a}$. Increased reliance on carbohydrate oxidation is seen during the brief interbout arousal periods and disruption of rewarming is seen when animals are given an inhibitor of glycolysis but not when given an inhibitor of lipid oxidation (Dark and Miller, 1997; Karpovich et al., 2009). Furthermore, levels of mitochondrial respiration were significantly 
increased as were activity measurements of succinate dehydrogenase during arousal from torpor, as compared to torpid animals, when measured in either liver or muscle (Armstrong and Staples, 2010; Brown et al., 2013), showing that in some organs, the favourability of lipid oxidation over carbohydrate oxidation may be somewhat limited to only the torpid stages of hibernation in these tissues. In fact, rewarming from torpor and metabolism during interbout arousals together account for the use of more than half of a hibernator's entire winter-time fuel store (French, 1985; Wang, 1979), although mechanisms are still likely in place to limit the uptake of circulating glucose by the body's organs from reaching summer-time levels during these periods (Andrews et al., 2009).

As would be expected following a sudden reversal of hypometabolism, mammalian hibernators must also find ways to protect tissues in the face of enormous increases in oxygen consumption and free-radical generation, as well as increased carbohydrate consumption following uncoupled cellular respiration in BAT and shivering thermogenesis in skeletal muscle (Carey et al., 2003; Kloner et al., 1998; Meyer et al., 2012). Increased levels of antioxidant defences are observed during arousal from hibernation including heme oxygenase 1, and associated effector proteins including nuclear factor (erythroid-derived 2)-like 2 (Nrf2) and the transcription factor MafG, in the liver, kidney, brain and heart of aroused 13LGSs (Ni and Storey, 2010). Antioxidant defences stemming from the transcriptional activity of nuclear factor kappalight-chain-enhancer of activated B cells (NF- $\mathrm{B}$ ) are also significantly increased in skeletal muscle tissue from 13LGSs transitioning to the arousal phase of the torpor/arousal cycle (Allan and Storey, 2012; Morin et al., 2008; Vucetic et al., 2013). In a similar fashion, anti-apoptotic protein expression is increased within a variety of tissues throughout the torpor cycle, as compared to summer euthermic animals (Fleck and Carey, 2005; Logan et al., 2016a; Rouble et al., 2013), as is heat shock protein expression (Feder and Hofmann, 1999; Storey and Storey, 2011; Vermillion et al., 2015).

Thanks to multiple cytoprotective mechanisms that are upregulated during arousal, 13LGSs may survive multiple bouts of torpor and subsequent arousal via protection from the 
harmful consequences of decreased tissue use (i.e., muscle atrophy), free-radical generation, as well as shifts in metabolic fuel or energy requirements and metabolite buildup or depletion. Since mammalian hibernators have these protective mechanisms, their abilities to defend themselves from cellular stresses that are atypical of normal mammalian life are of great interest to human medical research - especially with regards to obesity and diabetes (Kirchner et al., 2013; Sookoian and Pirola, 2013; Wu et al., 2013), aging and longevity (Storey and Storey, 2004a; Wu and Storey, 2016) and neurodegenerative damage or diseases (Drew et al., 2007; Logan et al., 2016b; Wood, 2015).

\section{Molecular Regulation of Mammalian Hibernation}

In order for the 13LGS to progress through the phases of the torpor/arousal cycle, control over a variety of cellular processes is required in the form of genomic control over transcription, protein synthesis, as well as covalent modification of metabolic enzymes and structural proteins (as previously described). Covalent modifications to mature proteins collectively are termed posttranslational modifications (PTMs) and fine tuning in this way can massively alter the modified protein's biological activity or even the protein's interactions, stability and subcellular location. Furthermore, compared to the cost of modulating gene expression, often times, PTMs are

cheaper, faster and much more energy-efficient. A vast array of proteins may be altered by PTMs, and in any one animal's cell, thousands of proteins may be displaying stable PTMs that are currently recognized by the literature. Also of interest, is the combinatorial nature of multiple PTMs which have the ability to act either additively or to cause interference with one another, either on single proteins or on multiple proteins, potentially altering the dynamics of protein interaction networks (Duan and Walther, 2015; Stram and Payne, 2016).

\section{Post-Translational Modifications Alter Cellular Mechanisms in Mammalian Hibernation}

In the case of mammalian hibernation, numerous examples of RPP are essential to the switch from glycolytic to lipid catabolism, and the almost-complete cessation of gene transcription and mRNA translation, and are critical to a wide variety of hypometabolic strategies 
(Storey and Storey, 2004b). For example, cellular energy in the form of ATP turnover during hibernation is extremely precious, and mechanisms for its protection are under the control of RPP in skeletal muscle, where phosphorylation of the $\mathrm{Na}^{+} \mathrm{K}^{+}$-ATPase and sarcoplasmic reticulum $\mathrm{Ca}^{2+}$-ATPase membrane ion pumps reduces their activity, limiting metabolic oxygen consumption and $\mathrm{CO}_{2}$ production (Bell et al., 2014; MacDonald and Storey, 1999). Several other examples of RPP's role in mammalian hibernation were also detailed in the previous section, including control over ribosomal initiation and elongation factors, as well as key glycolytic and lipogenic enzymes (Andrews et al., 1998; Frerichs et al., 1998; Storey, 1987; 1997).

While the majority of focus in hibernator research with regard to PTMs lies within RPP and the associated kinases and phosphatase enzymes that control it, other protein covalent modifications have not been as extensively characterized including reversible protein acetylation (RPA) and reversible protein methylation (RPM). One of the first studies of the role of RPA in 13LGS torpor/arousal cycles was completed in our lab, and found that levels of histone deacetylase (HDAC) were increased during torpor in muscle tissue (Morin and Storey, 2006). Building on this work, protein amounts activity levels of sirtuins (SIRTs), enzymes that deacetylate modified proteins, were assessed and found to show increased levels of SIRT3, protein, decreased acetylation on the SIRT3 downstream target superoxide dismutase-2, and heightened total SIRT enzymatic activity (Rouble and Storey, 2015). Given the tendency of acetylation modifications to increase cellular activity levels, decreased protein acetylation via increased levels of deacetylase enzymes would serve to preserve cellular energy levels and inhibit a variety of distinct cellular pathways.

Another extremely relevant modification occurs when enzymes that modulate RPP or RPA target histone proteins, the proteins which wrap DNA around themselves to facilitate DNA packing and bending. The nucleosome complex is the name given to the $\sim 146$ base pairs of DNA, wrapped around an octamer of two copies each of histone $\mathrm{H} 2 \mathrm{~A}, \mathrm{H} 2 \mathrm{~B}, \mathrm{H} 3$ and $\mathrm{H} 4$ proteins as well as the linker histone H1. The nucleosome has the ability to compact DNA by about 40 -fold, as 
well as to change the DNA molecule via deviations from linearity (Luger et al., 1997; MariñoRamírez et al., 2005). In addition, modifications of the N-terminal and C-terminal amino acid tails that protrude from the nucleosome complex can cause further conformational changes to DNA shape and compaction, ultimately altering the accessibility of DNA-binding proteins and transcriptional machinery to the DNA molecule (Chew et al., 2006; Morales and Richard-Foy, 2000). A large array of modifications including phosphorylation, acetylation, methylation, ubiquitylation, neddylation, SUMOylation, etc., can target histone tails and histone modifications are typically classified as either activating or inhibitory towards gene expression, although this classification ignores the histone code which takes into account the fact that modifications to histone tails act in concert to alter DNA accessibility and packing (Bernstein et al., 2007; Izzo and Schneider, 2010). This cellular mechanism is the basis of epigenetic regulation, heritable and environmentally-modulated reversible changes in gene expression that are mediated by mechanisms that do not affect the underlying DNA sequence. As an example, both RPP and RPA can target histone tails, and this regulation is manipulated during the torpor arousal cycle (Morin and Storey, 2009). Levels of the activating marks - phosphorylation of histone H3 at serine 10 (H3S10p) and acetylation at lysine $23(\mathrm{H} 3 \mathrm{~K} 23 \mathrm{ac})$ - were reduced during hibernation (Morin and Storey, 2006), likely contributing to the suppression of DNA transcription during torpor.

\section{The role of protein methylation in altering cellular mechanisms}

The fact that PTMs have essential roles in the regulation and maintenance of mammalian torpor and arousal is clear, and this makes the potential investigation of the role of RPM in 13LGS hibernation a promising pursuit. The role of RPM has not been previously studied within the context of mammalian hibernation, however much attention has been paid to its role in the maintenance of genomic regulation - specifically epigenetic regulation.

The process of RPM transfers a methyl group (-CH3) to a target protein on either a lysine or arginine residue, typically from an S-adenosyl methionine (AdoMet) cofactor. When compared to the negatively charged phospho- or acetyl- functional groups, methyl modifications are 
relatively small and 'neutral,' causing only slight steric interference and no changes in the protein's charge. Also in contrast to RPP and RPA is the ability of methyltransferase enzymes to stack methyl- modifications, where lysine methyltransferases (KMTs) may create mono-, di-, or trimethylation marks on the $\varepsilon$-amino group of lysine residues, whereas arginine methyltransferases (PRMTs) may leave mono- or dimethylation marks (either symmetric or asymmetric) on its side chain amino group (Bannister and Kouzarides, 2011; Lan and Shi, 2009). The majority of RPM research has up until now been dominated by lysine methylation and the research in this thesis will similarly investigate lysine methyltransferases (KMTs) specifically.

The mechanisms of RPP, RPA and RPM are all relatively similar, and this can be seen in the ways that modifications are targeted to proteins and cause their effects, summarized by the 'writer-reader-eraser' model (Biggar and Li, 2015; Falkenberg and Johnstone, 2014). This model states that PTMs are written by a modifying enzyme, e.g., tyrosine kinase or histone acetylase, and that those PTMS are then read by an interacting effector protein, e.g., SH2 domains recognize phosphotyrosine whereas bromodomains recognize acetyl-lysine residues (Gamsjaeger et al., 2011; Marengere et al., 1994). Lastly, the modification may be erased by enzymes specific to their removal of PTMs, e.g., phophotyrosine phosphatases or histone deacetylases, for example, at the conclusion of whatever environmental stimuli signaled a need for a PTM in the first place. Drawing parallels, lysine methylation is accomplished through the activity of KMTs, and methyl-lysine modifications can be read by proteins from the chromodomain family or by proteins from the malignant brain tumor family of chromatin readers, among others. Lastly, methyl- modifications are removed through the action of lysine demethylases (KDMs) (Boccuni et al., 2003; Gu and Lee, 2013; Sims et al., 2005).

One mechanism by which RPM can regulate proteins is through competition for the same target residue, since both RPA and RPM target protein lysine residues. For example, lysine 299 (K299) on the transcription factor GATA4 can be either methylated by enhancer of zeste homolog 2 (EZH2), or acetylated by p300. During myocardial hypertrophy, GATA4(K299ac) is 
essential to the activation of cell-cycle gene activation and subsequent cardiogenesis (Takaya et al., 2008; Trivedi et al., 2010), however this modification is absent during normal cellular physiology. Methylation of the same lysine residue blocks acetylation by p300 and ultimately represses transcriptional activity. This finding is further explained by the observation that similar transcriptional repression occurs as the result of K299 mutation (He et al., 2012) showing that mechanisms for the attenuation of transcriptional activity are likely just as important as those that have the opposite effect. Similarly, the addition of a methyl group to a lysine residue may interfere with binding or allosteric regulation by directly blocking the associated interactions, as evidenced by transcription factor inaccessibility to the inactivated $\mathrm{X}$-chromosome in females via histone methylation (Heard, 2005; Panning and Jaenisch, 1998; Plath et al., 2003). Methylation can even affect a protein's subcellular localization as in the case of the chaperone protein, heat shock protein 70 (HSP70), which localizes to the cell's nucleus following dimethylation to produce HSP70(K572me ${ }^{2}$ ) by SET domain-containing protein 1A (SET1A), in contrast to unmodified HSP70 which remains in the cytoplasm during mitosis (Biggar and Li, 2015; Cho et al., 2012). Another effect that RPM may have is to change a protein's stability, as in the case of estrogen receptor alpha which requires lysine methylation for its correct recruitment to target genes (Subramanian et al., 2008), or myosin phosphatase target subunit 1, whose methylationinduced stabilization decreases levels of phosphorylated retinoblastoma protein and enhances cell cycle progression in cancer cells (Cho et al., 2011). One of the most interesting ways that RPM has been seen to affect cellular regulation is through interactions with other PTMs, wherein a protein's methylation status may affect its acetylation or phosphorylation or vice-versa (Biggar and Li, 2015; Zhang et al., 2015). An elegant example of the interaction of a methylation modification with another PTM is displayed within the C-terminal regulatory region of cellular tumor antigen p53 (p53). Under normal cellular physiology, p53 is ubiquitylated and targeted for proteasomal degradation, however, genotoxic cellular stress activates the transcriptional activity of p53 which regulates important cellular processes including apoptosis, cell-cycle control and 
DNA repair, which is in part regulated by RPM (Gervin et al., 2003; Zhang et al., 2015). Methylation of $\mathrm{p} 53(\mathrm{~K} 370 \mathrm{me})$ or dimethylation of $\mathrm{p} 53\left(\mathrm{~K} 373 \mathrm{me}^{2}\right)$ is linked to transcriptional inhibition, whereas methylation of the nearby p53(K372me) has an opposite effect on p53's transcriptional activity and actually inhibits methylation of K370 and K373 (Chuikov et al., 2004; Huang et al., 2010; 2006). Other types of PTMs may also be influenced by the methylation status of these regulatory lysine residues and the crosstalk that occurs between modifications explains the molecular mechanism by which a number of transcription factors are able to quickly regulate their activity (Stark et al., 2010; Zhang et al., 2015). For instance, dimethylation of p53K370 and p53K382 and recruitment of the PHD finger-containing protein 20 reader protein are able to block ubiquitylation by the E3 ubiquitin ligase mouse double minute 2 homolog (Mdm2) and proteasomal degradation (Cui et al., 2012; Zhang et al., 2015). As mentioned previously, the crosstalk that takes place between PTMs on histone proteins is referred to as the histone code, and alters the conformation of chromatin into either 'relaxed' or 'compact' states (Bannister and Kouzarides, 2011; Kouzarides, 2007). In regards to RPM of H3, for example, highly active genes are located in the more accessible heterochromatin and are associated with $\mathrm{H} 3 \mathrm{~K} 4 \mathrm{me} / \mathrm{me}^{2} / \mathrm{me}^{3}$, H3K27me and H3K36me , among other PTMs, while silent genes are found in euchromatin which is associated with $\mathrm{H} 3 \mathrm{~K} 9 \mathrm{me}^{2}$ and either $\mathrm{H} 3 \mathrm{~K} 27 \mathrm{me}^{2}$ or $\mathrm{H} 3 \mathrm{~K} 27 \mathrm{me}^{3}$ (Barski et al., 2007; Plath et al., 2003; Rosenfeld et al., 2009; Santos-Rosa et al., 2002; Snowden et al., 2002).

It is clear that the diverse roles and functions of RPM make this modification a prime candidate for regulating a wide variety of cellular processes and mechanisms including chromatin dynamics, signal transduction and metabolic pathways, and protein interaction networks (Biggar and Li, 2015; Hamamoto et al., 2015a; Zhang et al., 2015). In fact, RPM is so widespread in regulating cellular processes that the AdoMet cofactor is actually the second most widely-used enzyme substrate consumed, behind ATP (Schubert et al., 2003). These reasons have presented KMT enzymes as interesting candidates for research on cellular physiology and homeostatic mechanisms. 
KMT enzymes are incredibly conserved and are found throughout the animal kingdom even occurring in bacteria (Cain et al., 2014; Calpena et al., 2015), highlighting that regulation by RPM plays a crucial role in a variety of subcellular processes. Furthermore, KMT enzymes are typically very specific in their substrate specificity. For instance, the methyltransferase SET domain-containing protein 7/9 (SET7/9) is only able to monomethylate proteins, and specifically only at lysine 4 of the histone $\mathrm{H} 3$ protein (H3K4me), whereas Suppressor of variegation 3-9 homolog 1 (SUV39H1) may either mono-, di- or trimethylate H3K9, e.g., (H3K9me/me $2 / \mathrm{me}^{3}$ ). The substrate specificity of KMT enzymes, which typically share a catalytic Suppressor of variegation 3-9 (Su(var)3-9), Enhancer of zeste, Trithorax (SET) domain, are the result of differences in an aromatic amino acid residue within the enzyme's active site that deprotonates a target protein lysine residue (Chin et al., 2006; Guo and Guo, 2007; Zhang et al., 2003).

Because KMT enzymes are so conserved and share a catalytic domain, grouping the enzymes into families is accomplished according to their homology and similarities in noncatalytic protein domains or their target specificity (particularly their interactions with histones), creating eight KMT families (Allis et al., 2007; Zhang et al., 2012a). Of relevance to the research in this thesis are the KMT1, KMT2, KMT3, KMT5 and KMT7 families (Reviewed in Table 1.1). KMT1 enzymes including SUV39H1 and G9a Histone Methyltransferase (G9a) all share a pre-SET and post-SET domain flanking their SET domain, and all target the H3K9 residue associated with transcriptional repression. On the other hand, all members of the KMT2 family, including Set1/Ash2 histone methyltransferase complex subunit ASH2 (ASH2L), share only a Cterminus post-SET domain and target the transcriptional activator H3K4 for methylation, although may only act in complex with other KMT enzymes including retinoblastoma binding protein-5 (RBBP5). Transcriptional activation is also a hallmark of KMT3 family members, e.g., SET and MYND domain-containing protein 2 (SMYD2), loosely joined by their lack of pre- and post- SET domains and their targeting of either H3K4 or H3K36 for monomethylation. Members of the KMT5 family, such as SET domain-containing protein 8 (SET8) target H4K20, a 
methylation marker of chromatin activity (i.e., DNA-replication and cell cycle progression, DNAdamage response, etc) but do not share significant sequence similarities. Finally, the KMT7 family consists of only one protein, SET domain-containing protein 7/9 (SET7/9), whose recognition of a consensus target sequence makes it unique among KMT enzymes in its monomethylation preference for non-histone targets (Allis et al., 2007; Zhang et al., 2012a).

\section{Objectives and Hypothesis}

Mammalian hibernation and its associated metabolic rate depression are characterized by an inhibition of a variety of cellular processes that allow the 13LGS to preserve bodily fuel stores, while increasing the activity and efficacy of cytoprotective strategies. Given the importance of both RPP and RPA to torpor and arousal cycles in the 13LGS, and the similarities in regulatory abilities, it would appear that RPM would also play an important role in mammalian hibernation. RPM is a regulator of a large number of cellular processes, and a number of the targets of RPP and RPA are also candidate targets for RPM. Given these facts the hypothesis presents itself:

\section{Hypothesis:}

RPM and changes to the abundance and activity of the enzymes that regulate this

\section{PTM are correlated with the rapid and reversible changes in cellular physiology that occur during mammalian hibernation in the 13LGS.}

To test this hypothesis, this thesis will investigate protein amounts and activities of several KMT enzymes as well as the methylation status of selected downstream targets in liver and muscle tissues of the 13LGS over several time points of the torpor/arousal cycle.

\section{Specific hypothesis 1:}

Skeletal muscle undergoes molecular changes in order to regulate alternating levels of cellular and physiological activity over the torpor/arousal cycles and must limit the amount of muscle atrophy that results from disuse during hibernation. KMT enzymes will show fluctuations in abundance and enzyme activity during hibernation. Similarly, the methylation of their downstream targets will change accordingly. 
Chapter 3 addresses this hypothesis by measuring the responses of selected methyltransferases (SMYD2, SUV39H1, SET8, SET7/9, G9a, ASH2L and RBBP5) in skeletal muscle tissue of the 13LGSs over a multi-point time course of torpor/arousal, as well as quantifying the total histone methyltransferase activity at H3K4, H3K9 and H3K27. The methylation status of selected target proteins in muscle is also measured including $\mathrm{H} 3 \mathrm{~K} 4 \mathrm{me}$, H3K9me ${ }^{3}$, H3K27me, H3K36me ${ }^{2}$ and heat shock protein 90 (HSP90me).

\section{Specific hypothesis 2:}

The 13LGS liver must regulate a major shift in metabolic strategy as well as the storage and use of glycogen reserves during hibernation. KMT enzymes will show differential abundance and fluctuations of activity over torpor/arousal cycles in liver during hibernation and their downstream targets will change accordingly.

Chapter 4 addresses this hypothesis by measuring the responses of SMYD2, SET8, SET7/9, G9a, ASH2L and RBBP5 in liver tissue of the 13LGS, as well as the total histone methyltransferase activity at $\mathrm{H} 3 \mathrm{~K} 4, \mathrm{H} 3 \mathrm{~K} 9$ and $\mathrm{H} 3 \mathrm{~K} 27$. The methylation status of $\mathrm{H} 3 \mathrm{~K} 4 \mathrm{me}$, $\mathrm{H} 3 \mathrm{~K} 9 \mathrm{me}^{3}, \mathrm{H} 3 \mathrm{~K} 36 \mathrm{me}^{2}$, p53K370me ${ }^{2}$ and p53K372me during hibernation will also be measured in this tissue.

Previous research, that was outlined above, has illuminated some of the roles that other PTMs have in regulating mammalian hibernation (Bell et al., 2014; MacDonald and Storey, 1999; Morin and Storey, 2006; Rouble and Storey, 2015), however the present thesis is the first investigation of the role of RPM in the regulation of mammalian hibernation. This research provides a unique starting point for characterizing the role(s) that RPM play in regulating mammalian hibernation in the 13LGS. Furthermore, this understanding will help to shine light on the way in which KMT enzymes are able to regulate metabolic adaptations that the animal must undertake in order to survive using a hypometabolic strategy of overwintering. 


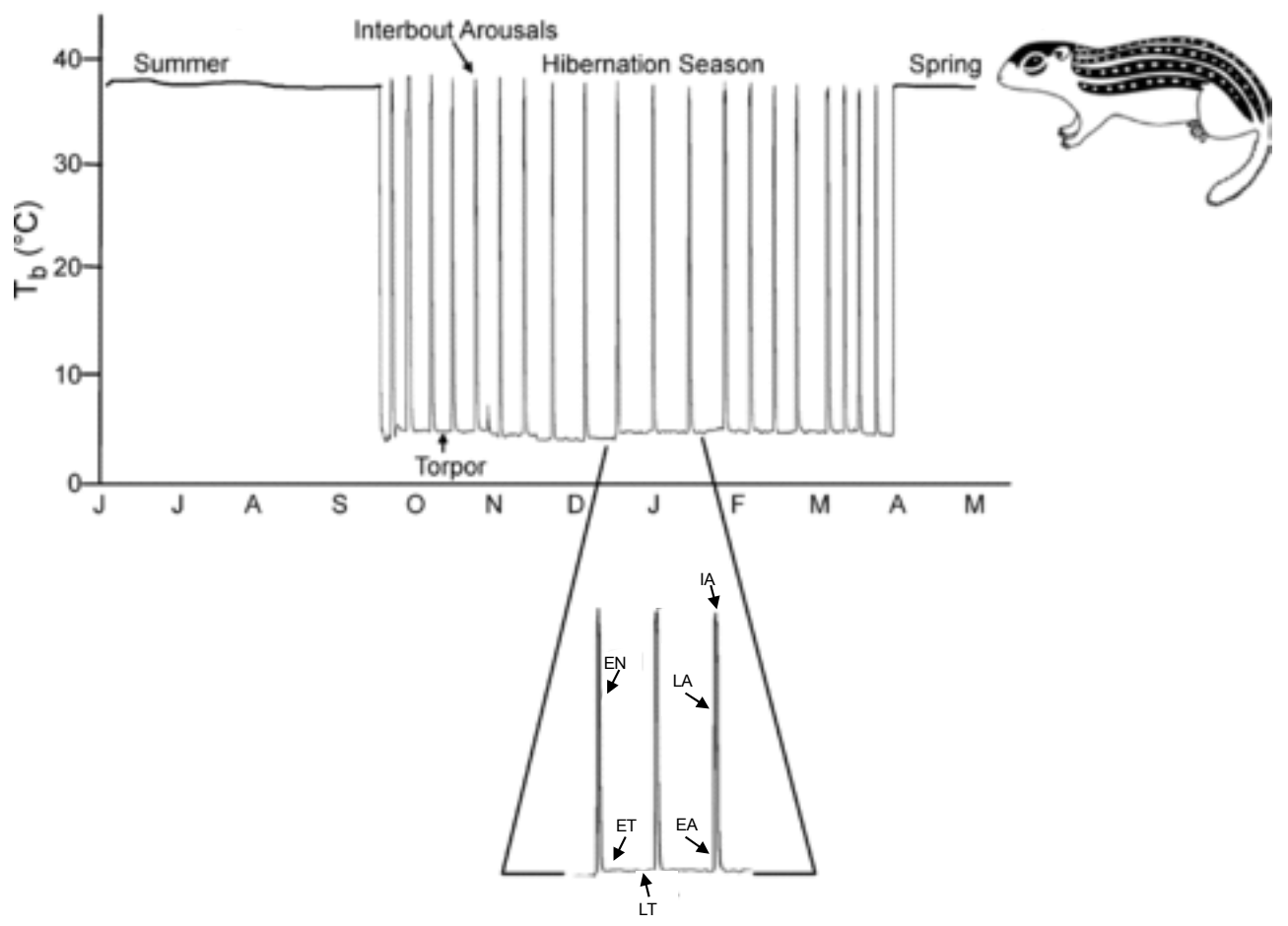

Figure 1.1: A representation of torpor/arousal cycles during hibernation in 13-lined ground squirrels (Ictidomys tridecemlineatus) is produced by plotting body temperature across the seasons, starting in June. Inset depicts the entrance into torpor (EN), early torpor (ET), late torpor (LT), early arousal (EA), late arousal (LA) and interbout arousal (IA) periods. (Image derived from Nelson et al., 2009) 
Table 1.1: Family and substrate specificity for KMT enzymes of relevance to this thesis

\begin{tabular}{llll}
\hline Family & Enzyme & $\begin{array}{l}\text { Main Histone Target } \\
\text { Residue* }\end{array}$ & $\begin{array}{l}\text { Non-Histone } \\
\text { Targets }\end{array}$ \\
\hline KMT1 & SUV39H1 & H3K9 & \\
KMT2 & ASH2L & H3K4 & HSP90, p53 \\
KMT3 & SMYD2 & H3K36 & p53 \\
KMT5 & SET8 & H4K20 & \\
KMT7 & SET7/9 & H3K4 & \\
N/A & RBBP5 & H3K4 & \\
\hline$*:$ Where KMT enzymes may modify more than one residue only the family-defining & & \\
\multicolumn{2}{r}{ residue is listed } & &
\end{tabular}




\section{Chapter 2}

\section{General Materials and Methods}




\section{A. Watts' Contribution Statement}

The procedures presented under the heading of "Animal Experiments" below were performed by researchers other than A. Watts. All remaining techniques, data collection, and data analysis outlined under all other headings within this Chapter were performed solely by A. Watts.

\section{Animal Experiments}

Wild-captured thirteen-lined ground squirrels (Ictidomys tridecemlineatus) were caught in late summer and autumn by a USDA-licenced trapper (TLS Research, Bartlett, IL), when the animals' body weight ranged from 150-180g. Experiments and procedures were carried out in the Animal Hibernation Facility of the National Institute of Neurological Disorders and Stroke and were approved by the Animal Care and Use Committee (NIH, Bethesda, MD). Experiments were carried out and described by the lab of Dr. J. M. Hallenbeck (Frerichs et al., 1994; 2010). Prior to hibernating and following a quarantine period, animals were housed in shoebox cages at ambient temperature $\left(21^{\circ} \mathrm{C}\right)$ on a 12 -hour light/dark cycle and had ad libitum access to standard rodent diet and water. Animals were subcutaneously-implanted with a sensor chip (IPTT-300; Bio Medic Data Systems, Seaford, DE) while anesthetized with 5\% isoflurane in order to allow measurements of $\mathrm{Tb}$ that define the different stages of torpor and arousal. Hibernation experiments took place during the winter months after the animals' lipid stores had accumulated enough to allow the squirrel to descend into torpor, at which point the animals were transferred to a hibernaculum with individual cages lined with wood shavings and maintained at $4-5^{\circ} \mathrm{C}, 60 \%$ humidity and constant darkness. Noise within the chamber was kept at a minimum level. Animals typically settled into their first torpor bout within three days.

Animals' hibernation phase was determined after squirrels had been through multiple torpor bouts; four male animals were used in each study group and categorized by their $T_{b}$ and duration of $\mathrm{T}_{\mathrm{b}}$ depression into one of several categories, described as follows:

1. Euthermic in the Cold room (EC): euthermic animals that had not descended into torpor for more than at least 72 hours and during which time, maintained their $\mathrm{T}_{\mathrm{b}}$ within 36- 
$37^{\circ} \mathrm{C}$. These animals were exposed to the same conditions as all other sample groups and were therefore chosen as the reference group.

2. Entrance into torpor (EN): describes the period where squirrels had begun to reduce their $T_{b}$ as they entered torpor; sampling was done when $T_{b}$ was within the range of 18$31^{\circ} \mathrm{C}$.

3. Early Torpor (ET): animals had entered torpor and were sampled when they had maintained a low $\mathrm{T}_{\mathrm{b}}\left(5-8^{\circ} \mathrm{C}\right)$ and breathing rate $(4-6$ breaths $/ \mathrm{min})$ for $\sim 24$ hours and no longer than five days.

4. Late Torpor (LT): animals were in continuous torpor with low $\mathrm{T}_{\mathrm{b}}\left(5-8^{\circ} \mathrm{C}\right)$ and breathing rate (4-6 breaths/min) for five days or more.

5. Early Arousal (EA): animals had begun the arousal phase and were sampled when $T_{b}$ was rising $\left(\sim 9-12^{\circ} \mathrm{C}\right)$ and respiratory rate had increased to $\sim 60$ breaths $/ \mathrm{min}$.

6. Late Arousal (LA): denotes the animals that reached $\mathrm{a} \mathrm{T}_{\mathrm{b}}\left(28-32^{\circ} \mathrm{C}\right)$ and respiratory rate that was close to euthermic values.

7. Interbout Active (IA): animals naturally aroused from torpor that had reestablished euthermic $\mathrm{T}_{\mathrm{b}}$ for at least 18 hours following a bout of torpor of at least 72 hours.

Animals from each of the preceding stages were anesthetized (5\% isoflurane) before sacrificing within two minutes of disturbance from their hibernaculum; samples of hind leg skeletal muscle tissue and liver tissue were dissected and frozen in liquid nitrogen. Once all samples were collected the tissues were shipped to Carleton University on dry ice and were immediately stored at $-80^{\circ} \mathrm{C}$.

\section{Preparation of Soluble Protein Extracts}

Frozen muscle and liver tissue from four individual animals at each time point of the torpor/arousal cycle were crushed using a mortar and pestle cooled in liquid nitrogen. Tissue homogenization with sonication was carried out in 1:2.5(w/v) ice-cold homogenizing buffer [20 mM 4-(2-hydroxyethyl)-1-piperazineethanesulfonic acid (HEPES) (pH 7.8), 200 mM NaCl, 0.1 
$\mathrm{mM}$ ethylenediaminetetraacetic acid (EDTA), $10 \mathrm{mM} \mathrm{NaF}, 1 \mathrm{mM} \mathrm{Na} \mathrm{VO}_{4}, 10 \mathrm{mM} \beta$ glycerophosphate] and using a Polytron P10 homogenizer. Immediately prior to homogenization $1 \mathrm{mM}$ PMSF (BioShop, Cat\#PMS123.25) and $1 \mu \mathrm{L}$ of protease inhibitor cocktail [104 mM AEBSF, $80 \mu \mathrm{M}$ aprotinin, $4 \mathrm{mM}$ bestatin, $1.4 \mathrm{mM}$ E-64, $2 \mathrm{mM}$ leupeptin, $1.5 \mathrm{mM}$ pepstatin A] (BioShop; Cat\#PIC001.1) were added to each sample. Samples were then centrifuged at 10,000 RPM for 15 minutes at $4^{\circ} \mathrm{C}$ and the resulting supernatant containing soluble protein was removed. In order to ensure a consistent amount of protein sample was being used for quantification, protein concentrations were then quantified via the Coomassie blue dye-binding assay using the BioRad reagent (BioRad Laboratories; Hercules, CA; Cat\#500-0006) with absorbance read at 595 $\mathrm{nm}$ on a PowerWave HT microplate spectrophotometer and associated Gen5 Microplate Reader and Imager Software (Version 1.09; BioTek, Winooski, VT) using bovine serum albumin as a relative standard. Homogenization buffer was added to standardize all samples to $10 \mu \mathrm{g} / \mu \mathrm{L}$. Protein extracts used for methyltransferase activity assays were completed at this step, and were stored at $-40^{\circ} \mathrm{C}$. Protein samples that were used for Western blotting required addition of $1: 1 \mathrm{v} / \mathrm{v}$ 2X SDS loading buffer [100 mM Tris-base ( $\mathrm{pH} 6.8$ ), 4\% w/v SDS, 20\% v/v glycerol, $0.2 \% \mathrm{w} / \mathrm{v}$ bromophenol blue, $10 \% \mathrm{v} / \mathrm{v} 2$-mercaptoethanol] to bring the final protein concentration in each sample to $5 \mu \mathrm{g} / \mu \mathrm{L}$. Samples were then boiled for five minutes to allow for protein denaturation and then stored at $-40^{\circ} \mathrm{C}$.

\section{Western Blotting}

Equal amounts of protein sample (10-50 $\mu \mathrm{g})$ were loaded onto either SDSpolyacrylamide gels or Tris-tricine gels within a Mini Protean III apparatus (BioRad; Missisauga, ON), along with PiNK Plus pre-stained protein ladder (10.5-175 kDa, FroggaBio, Cat\#PM0050500). Experiments were run using independent biological replicates (extracted from multiple animals) and using multiple runs for each target.

KMT enzymes and non-histone protein targets were separated using SDS-polyacrylamide gels which can be described as a discontinuous system where the stacking gel $[0.5 \mathrm{M}$ Tris- $\mathrm{HCl}$ 
(pH6.8), 4\% (w/v) acrylamide, $0.4 \%(\mathrm{w} / \mathrm{v})$ sodium dodecyl sulfate (SDS), $0.1 \%(\mathrm{w} / \mathrm{v})$ ammonium persulfate (APS), and $0.001 \%(\mathrm{v} / \mathrm{v}) \mathrm{N}, \mathrm{N}, \mathrm{N}$, N'-tetramethylethylenediamine (TEMED)] was buffered to $\mathrm{pH}$ 6.8, while the lower resolving gel [components for a 10\% gel: $0.4 \mathrm{M}$ Tris-base (pH8.8), 10\% (w/v) acrylamide, 0.1\% (w/v) SDS, 0.1\% (w/v) APS, and 0.001\% (v/v) TEMED] was buffered to $\mathrm{pH}$ 8.8. SDS-PAGE was performed at $180 \mathrm{~V}$ for $45-180$ minutes in $1 \mathrm{X}$ Trisglycine SDS running buffer [25 mM Tris (pH8.3), $245 \mathrm{mM}$ glycine, 0.1\% (w/v) SDS]. Histone protein targets were separated using a Tris-tricine PAGE system where both the stacking gel [0.75 M Tris-HCl/SDS ( $\mathrm{pH} 8.45), 4 \%(\mathrm{w} / \mathrm{v})$ acrylamide, 0.1\% (w/v) APS, and 0.001\% (v/v) TEMED] and resolving gel [1.0 M Tris-HCl/SDS (pH 8.45), 15\% (w/v) acrylamide, $0.1 \%(\mathrm{w} / \mathrm{v})$ APS, and $0.001 \%(\mathrm{v} / \mathrm{v})$ TEMED] components were buffered to $\mathrm{pH}$ 8.45. Tris-tricine PAGE was run at $60 \mathrm{~V}$ for 25 minutes followed by $\sim 2$ hours at $160 \mathrm{~V}$ using separate anode buffer $[0.2 \mathrm{M}$ Tris- $\mathrm{HCl}(\mathrm{pH} 8.8)]$ and running buffer [0.1 M Tris- $\mathrm{HCl}(\mathrm{pH} 8.3), 0.1 \mathrm{M}$ tricine, $1 \% \mathrm{w} / \mathrm{v}$ SDS]. Transfer of proteins onto a $0.45 \mu \mathrm{m}$ PVDF membrane was accomplished by electroblotting using transfer buffer containing $25 \mathrm{mM}$ Tris ( $\mathrm{pH} 8.5$ ), $192 \mathrm{mM}$ glycine and 10\% v:v methanol; conditions for transfer were either i) $160 \mathrm{~mA}$ for 1-3 hours at room temperature for SDSpolyacrylamide gels or, ii) $30 \mathrm{~V}$ for 2 hours at $4{ }^{\circ} \mathrm{C}$ for Tris-tricine polyacrylamide gels.

After electroblotting was complete, PVDF membranes were washed with Tris-buffered saline with Tween-20 (TBST) (20 mM Tris-base (pH 7.6), $140 \mathrm{mM} \mathrm{NaCl}$, and 0.05\% (v/v) Tween-20) and then were blocked with skim milk powder $(0.5 \%-8.0 \%(\mathrm{w} / \mathrm{v}))$ dissolved in TBST for 10-30 minutes on a rocker at room temperature. Blotted and blocked membranes were washed three times with TBST and then incubated with specific primary antibodies $(1: 1000 \mathrm{v} / \mathrm{v}$ diluted in TBST) at $4{ }^{\circ} \mathrm{C}$ overnight. Purchased antibodies, which typically target human orthologues of proteins in the thirteen-lined ground squirrel proteome, were confirmed to correspond to sites on the orthologous squirrel protein (as shown in Appendix A; a list of the primary antibodies used is available in Appendix B). Three washes were performed in TBST before incubation with HRPlinked anti-rabbit or anti-mouse IgG secondary antibody (1:6000 to 1:8000 v/v dilution in TBST) 
for 20-40 minutes at room temperature, followed by a final three washes in TBST. Specific protein bands were visualized via enhanced chemiluminescence $\left(1: 1 \mathrm{H}_{2} \mathrm{O}_{2}\right.$ and Luminol) and the ChemiGenius BioImaging System (Syngene, Frederick, MD). Membranes were then stained using Coomassie blue $(0.25 \% \mathrm{w} / \mathrm{v}$ Coomassie brilliant blue, $7.5 \% \mathrm{v} / \mathrm{v}$ acetic acid, $50 \%$ methanol) to visualize all protein present in each lane. In order to account for potential minor variations in sample loading between different lanes, the densities of immunoreactive protein bands corresponding to the protein of interest were standardized against the summed intensity of a group of Coomassie stained bands in the same lane (Eaton et al., 2013); these were constant across all lanes and distinctly different from the immunoreactive band of interest. When necessary, Western blots were re-run to overcome problems with technical problems due to sample or analysis failures; when problems were due to sample failure these experiments used additional independent biological replicates. Immunoreactive bands were not further characterized using mass spectrometry due to the techniques insensitivity to low-abundance proteins and the requirement of further purification.

\section{Methyltransferase Activity Assays}

In order to determine the relative activity of lysine methyltransferases in control, torpid and aroused ground squirrels, a series of EpiQuick Histone Methyltransferase Activity/Inhibition Assay Kits from Epigentek (Farmingdale, NY) were employed that measure lysine methyltransferase (KMT) activity at specific sites on histone H3, namely, methylation target sites at lysine-4 (K4; Cat\#P-3017), lysine-9 (K9; Cat\#P-3018) and lysine-27 (K27; Cat\#P-3020). Total soluble protein extracts were carried out using the procedure detailed under Preparation of total soluble protein extracts for liver and skeletal muscle tissue from individual animals sampled from four out of the six time-points of the torpor/arousal cycle (EC, EN, LT, IA; N=4). Total soluble protein extracts were normalized to a concentration of $10 \mu \mathrm{g} / \mu \mathrm{L}$ by addition of small volumes of homogenizing buffer. 
A standard curve and dilution curve were completed before results were collected for validation of the assays; relevant information can be found in Appendix C. All components of the assay, except for protein samples, were supplied by the manufacturer. According to manufacturer's instructions, $1.5 \mu \mathrm{L}$ of AdoMet solution (5X stock solution provided), $3 \mu \mathrm{L}$ of biotinylated histone substrate, $24 \mu \mathrm{L}$ of histone assay buffer and $3 \mu \mathrm{L}$ of protein samples were added to each reaction well of the microplate. The microplate was then covered with Parafilm M and incubated at $37^{\circ} \mathrm{C}$ for one hour on an orbital shaker, followed by aspiration and washing of each well three times with $150 \mu \mathrm{L}$ of $1 \mathrm{X}$ wash buffer ( $\mathrm{pH} 7.2-7.5 ; 10 \mathrm{X}$ stock solution provided). The reaction wells were then incubated with $50 \mu \mathrm{L}$ of 1:100 capture antibody (manufacturer provided at $100 \mu \mathrm{g} / \mathrm{mL}$ ) for one hour on an orbital shaker at room temperature. The wells were then washed four times (H3-K27) or five times (H3-K4 \& H3-K9) with $150 \mu \mathrm{L}$ of $1 \mathrm{X}$ wash buffer before the addition of $50 \mu \mathrm{L}$ of 1:1000 detection antibody (manufacturer provided at 100 $\mu \mathrm{g} / \mathrm{mL}$ ) for 30 minutes on an orbital shaker at room temperature. A final four washes (H3-K27) or five washes (H3-K4 \& H3-K9) with $150 \mu \mathrm{L} 1 \mathrm{X}$ wash buffer were completed before adding $100 \mu \mathrm{L}$ of developing solution. The microplate was then incubated in the dark for ten minutes at room temperature before the addition of a $50 \mu \mathrm{L}$ aliquot of stop solution. Absorbance in each reaction well was immediately measured at $450 \mathrm{~nm}$ using a PowerWave HT microplate spectrophotometer and associated Gen5 Microplate Reader and Imager Software (Version 1.09; BioTek, Winooski, VT). A control well containing all components except protein samples was run concurrently during the assay and was used as a negative control 'blank' value during data analysis, as per the manufacturer's instructions.

In order to relate OD values to KMT activity at a specific methylation target site, an equation (provided by the manufacturer) was used to yield activity values in appropriate units (ng methyllysine produced per hour per mg protein added), and can be found in Appendix C. 


\section{Quantification and statistics}

Band densities on chemiluminescent immunoblots were visualized using a Chemi-Genius BioImaging system (Syngene, Frederick, MD) and quantified using the accompanying GeneTools software, version 4.02. The density of immunoblot bands were normalized against the summed intensity of a group of Coomassie stained protein bands in the same lane as the band of interest, with the group of Coomassie bands chosen for a lack of variability between control and experimental states and physical separation from the immunoreactive band of interest. Statistical analysis of the differences in relative total soluble protein abundance between experimental timepoints was performed using a one-way ANOVA and post-hoc testing (Tukey, $\mathrm{p}<0.05$ ) using SigmaPlot 12 with $\mathrm{p}<0.05$ accepted as significant.

Relative KMT enzyme activity was calculated from the corrected optical density (sample OD minus OD of blank wells without protein), and expressed as nanograms of methyllysine produced per hour per mg protein $(\mathrm{ng} / \mathrm{h} / \mathrm{mg})$. Statistical analysis was done to compare KMT activity between experimental time-points using a one-way ANOVA and post-hoc testing (Tukey, $\mathrm{p}<0.05$ ) using SigmaPlot 12 with $\mathrm{p}<0.05$ accepted as significant. 


\section{Chapter 3}

\section{Analysis of Histone Methyltransferase Enzyme Abundance and their Downstream Targets in Skeletal Muscle during Mammalian Hibernation}




\section{Introduction}

Skeletal muscle is the striated contractile muscle responsible for locomotion under voluntary control, as well as a number of involuntary movements including breathing. Another role of skeletal muscle tissue is the generation of heat through shivering thermogenesis, a function that has an important role for mammalian hibernation (Lee et al., 2008; Shavlakadze and Grounds, 2006; Wu et al., 2013). Because the proper functioning of skeletal muscle is vital to an animal's existence, either increases (hypertrophy) or decreases (atrophy) in muscle mass are consequential to the animal's overall metabolism and its survival; for instance, in hibernation, the ability to fend off the molecular pathways that lead to muscular disuse are vital for the animal's functioning during spring-time arousal behaviors as well as to thermal generation during torpor.

As introduced in Chapter 1, hibernation provides small mammals with an interesting and unique strategy for overwintering; animals preload their bodies with large amounts of stored fuel reserves (acquired in late summer), abandon homeothermy to let $\mathrm{T}_{\mathrm{b}}$ sink to near-ambient temperatures and strongly depress metabolic rate so as to survive for many months without feeding until conditions are again conducive for active life (Klug and Brigham, 2015; Storey, 2010). In skeletal muscle of the 13LGS during torpor, decreases in activity, protein synthesis and nutrient intake all increase the potential for skeletal muscle disuse atrophy to occur, requiring cytoprotective mechanisms to intervene (Cotton, 2016; Ivakine and Cohn, 2014; Shavlakadze and Grounds, 2006). However, studies within hibernators have found little to no atrophy of skeletal muscle even though physiological activity is reduced to the point of weeks of inactivity (Lee et al., 2008; Nowell et al., 2011; Rourke et al., 2004). The persistence of skeletal muscle morphology is enhanced in mammalian hibernators through increased oxidative fiber expression and reduced protein degradation, so that muscle's oxidative capacity and protein levels are conserved during hibernation. In fact, the ratio of muscle mass to body mass may actually increase during hibernation, while muscle fiber cross-sectional area is relatively unaltered (Hindle et al., 2015; Yang et al., 2014). Findings that show that hibernating species may survive months 
of relative muscle disuse make hibernators effective model systems for understanding mechanisms of muscle morphology preservation that could prove beneficial to humans undergoing muscle disuse atrophy as a result of immobility, pathological effects or even weightlessness in space.

Furthermore, mammalian hibernators require regulation over sarcoplasmic activity for the use of shivering thermogenesis during arousal. In early arousal, blood flow to the limbs is limited in favor of oxygen supply to adipose tissues and sites of uncoupled respiration, but is restored in late arousal when the use of shivering thermogenesis for rewarming is heightened. Shivering thermogenesis in mammalian hibernators takes the form of several minutes of synchronous muscle fiber contractions, and requires the aerobic oxidation of fuels and proton gradient buildup across the mitochondrial membrane to provide energy for the electron transport chain and oxidative phosphorylation (Boyer and Barnes, 1999; Foster and Frydman, 1979; Samec et al., 1998). Muscle contractions are powered by the ATP-consuming activity of sarcoplasmic myosin, and therefore require increased oxygen delivery, facilitated by upregulated levels of myoglobin during the autumn and winter seasons which promote the use of oxygen dependent processes during arousal when myoglobin's $\mathrm{O}_{2}$ affinity decreases as a result of rising temperatures and the blood's partial $\mathrm{O}_{2}$ pressure is lowest (Fahlman et al., 2000; Masuda et al., 2013; Postnikova et al., 1999). A further requirement for shivering thermogenesis is the protection of the sarcomere and its morphology (Taylor-Burt et al., 2015). A regulatory mechanism involving RPM is required for this protection, in the form of SMYD2-mediated methylation of heat-shock protein 90 (HSP90), which causes complex formation of SMYD2, HSP90 with the N2A domain of titin and protection of the sarcomeric I-band. This has been demonstrated in non-hibernating mammalian species and deficiencies of this process result in titin degradation and impairments of muscle function (Donlin et al., 2012; Voelkel et al., 2013).

Given the need for functional skeletal muscle tissue during certain periods of the torpor/arousal cycle, whether for the synthesis of cytoprotective proteins or the generation of heat 
for the animal, it is clear that the extent of metabolic rate depression during torpor may not be as extensive as that seen in other tissues. In spite of factors which promote the use of skeletal muscle throughout winter, fluctuations in loading conditions, nutrient and resource supply, and environmental conditions show that an adaptive plasticity is necessary to survive the stresses associated with bouts of torpor and arousal (Tessier and Storey, 2016). Therefore, the role of histone PTMs in modulating and regulating gene expression as well as the role of non-histone PTMs in altering the activity and function of cellular proteins, likely serve important roles in the coordination of muscle cell-specific plasticity and cytoprotective mechanisms that are required during hibernation. For instance, investigations into the role of histone acetylation in controlling myogenic differentiation factors in non-hibernators have shown that their transcriptional activity is also dependent on a disassociation with histone deacetylase enzymes and concurrent association with and acetylation by histone acetyltransferase enzymes (Cao et al., 2005; Mal et al., 2001; Puri et al., 1997). Given an overlap in the functions of RPA and RPM, in that both regulate the expression of tissue-specific genes and transcription factors (Lau et al., 2004; Zhang and Zhang, 2011), it would not be surprising if differential protein levels of KMT enzymes or differential targeting of methyltransferase activity was seen during torpor/arousal cycles.

In order to properly reprioritize cellular functions while maintaining essential homeostatic mechanisms, a molecular mechanism of transcriptional and cellular regulation is required and RPM may fit this role. This chapter explores the changes in protein levels of the KMT enzymes, SET and MYND domain-containing protein 2 (SMYD2), Suppressor of variegation 3-9 homolog 1 (SUV39H1), SET domain-containing protein 8 (SET8), SET domaincontaining protein 7/9 (SET7/9), G9a Histone Methyltransferase (G9a), Set1/Ash2 histone methyltransferase complex subunit ASH2 (ASH2L) and Retinoblastoma-binding protein 5 (RBBP5) over the course of the torpor/arousal cycle in 13LGS skeletal muscle. In addition, the activity of KMT enzymes on relevant H3 residues, namely lysine-4 (H3K4), lysine-9 (H3K9) and lysine-27 (H3K27), were assessed to characterize relative capacities for histone methylation 
during hibernation in skeletal muscle. Methylation of histone lysine residues was also investigated for the monomethylated H3K4me, H3K27me, and dimethylated lysine-36 $\left(\mathrm{H} 3 \mathrm{~K} 36 \mathrm{me}^{2}\right)$ modifications. Finally, the methylation status of the SMYD2 downstream target, HSP90, was evaluated throughout the torpor/arousal cycle with a focus on the LA time point, during which time the animal is most reliant on sarcomeric activity for shivering thermogenesis. The results suggest roles for KMT enzymes in the regulation of torpor and arousal in the 13LGS and provide evidence for the role of RPM regulatory roles in mammalian hibernation.

\section{Materials and Methods}

\section{Animal experiments and total soluble protein extractions}

Animal experiments and total soluble protein extractions were conducted as described in

\section{Chapter 2.}

\section{Western blotting}

Western blotting was performed as described in Chapter 2. Relative protein levels of the studied KMTs (SMYD2, SUV39H1, SET8, SET7/9, G9a, ASH2L and RBBP5) and the downstream targets (H3K4me, H3K27me, H3K36me ${ }^{2}$, HSP90, and HSP90me) were assessed in total soluble protein extracts of skeletal muscle from animals at different phases of the torpor/arousal cycle. Antibodies used were: anti-SMYD2, anti-SUV39H1, anti-SET8, antiSET7/9, anti-G9a, anti-ASH2L, anti-RBBP5, anti-H3(K4me), anti-H3(K27me), antiH3(K36me ${ }^{2}$ ), anti-HSP90, anti-methyllysine (please refer to Appendix B for full details on each antibody). For a detailed list of the Western blotting conditions used for each protein target please refer to Appendix D.

\section{Total histone methyltransferase activity assay}

Total histone methyltransferase activity specific to H3K4, H3K9 and H3K27 residues were assayed in total soluble protein extracts from skeletal muscle using the EpiQuick Histone 
Methyltransferase Activity/Inhibition Assay Kit from Epigentek (Farmingdale, NY), as described in Chapter 2.

\section{Quantification and Statistics}

Quantification and statistics were carried out as described in Chapter 2.

\section{Results}

\section{Analysis of skeletal muscle KMT protein levels across the torpor/arousal cycle}

The relative protein levels of SUV39H1, SMYD2, SET8, SET7/9, G9a, ASH2L and RBBP5 in skeletal muscle were compared across the seven time points of the torpor/arousal cycle. Antibodies for each of the proteins (Appendix A) reacted with the appropriate number of bands (dependent on the presence of isoforms) at the expected molecular mass.

Data for SUV39H1, SMYD2, SET8, and SET7/9 are shown in Figure 3.1. Protein levels of SUV39H1 were not significantly different from EC values during entrance into torpor (EN) or during early torpor (ET) or late torpor (LT) periods (see expanded definitions of hibernation stages in Chapter 2). However, SUV39H1 levels rose significantly when animals entered the arousal phase. During early arousal (EA) and late arousal (LA), levels of SUV39H1 protein were significantly higher than ET levels by $1.7 \pm 0.03$ fold and $1.8 \pm 0.07$ fold respectively, whereas interbout arousal (IA) levels were significantly elevated compared to both ET $(1.9 \pm 0.14$ fold $)$ and LT $(1.6 \pm 0.14$ fold $)$. Levels of SMYD2 protein were generally not significantly different than EC levels, except during LT when values were just $0.4 \pm 0.04$ of EC values; SMYD2 levels were also significantly reduced as compared with both EN levels and ET maximum levels. During the arousal period SMYD2 protein levels rose again, although were still significantly decreased by about one-half as compared to the ET maximum. However, SMYD2 levels returned to control values during IA. Levels of SET8 showed no significant differences across the entire torpor/arousal cycle. Protein levels of SET7/9 increased during EN to $1.6 \pm 0.14$ fold higher than EC values and remained significantly elevated during torpor (e.g., LT was $1.6 \pm 0.02$ fold higher 
than EC). However, during EA, SET7/9 protein levels decreased by one-half as compared to EN, ET and LT values and were not significantly different than EC values ( $0.9 \pm 0.07$ of EC levels). During LA, SET7/9 protein levels rose again to $1.8 \pm 0.11$ fold higher than EC levels and were $2.0 \pm 0.11$ fold higher than the EA minimum levels; levels remained significantly elevated during IA (1.6 \pm 0.14 fold higher than EC levels).

Data for G9a, ASH2L and RBBP5 are shown in Figure 3.2. Protein levels of G9a were elevated specifically during two stages, rising $2.6 \pm 0.24$ fold in ET and $2.6 \pm 0.33$ fold in EA as compared with EC. At all other stages, G9a levels were not significantly different than EC values. Protein levels of ASH2L were strongly elevated by $3.6 \pm 0.21$ fold in ET as compared with EC, but then decreased by one-half in LT $(0.5 \pm 0.06$ fold compared to the ET maximum). During arousal, ASH2L protein levels rose again to $2.2 \pm 0.25$ fold higher in EA as compared with EC and continued to increase as arousal progressed; values in LA were $2.6 \pm 0.14$ fold higher and in IA were $3.2 \pm 0.19$ fold higher than EC levels. Protein levels of RBBP5 were elevated $1.9 \pm 0.05$ fold higher than EC in ET, protein levels then fell in LT to control values (falling $0.6 \pm 0.07$ fold compared to ET protein levels). RBBP5 protein levels were also increased during IA; RBBP5 levels were $2.2 \pm 0.17$ fold higher than EC values, and IA levels were significantly increased over all other time points except for ET.

\section{Assay of total histone methyltransferase activity in skeletal muscle}

Total histone methyltransferase activity was assessed and analyzed using total soluble protein extracts from skeletal muscle samples, in order to compare the methyltransferase activity present in samples taken from the EC, EN, LT and IA periods of the torpor/arousal cycle. Histone-lysine specific assays were employed for comparisons of methyltransferase activity at lysine residues K4 (H3K4), K9 (H3K9) and K27, (H3K27). Relative methyltransferase activity at $\mathrm{H} 3 \mathrm{~K} 4$ rose significantly in EN to $2.6 \pm 0.6$ fold higher than EC activity levels, but both other time points showed no change from EC levels. Relative methyltransferase activity at H3K9 in LT decreased $0.03 \pm 0.01$ fold compared to EC levels, although not different from EN or IA levels of 
activity. Finally, relative methyltransferase activity at H3K36 showed no significant differences from EC or from any other time points.

\section{Analysis of the methylation status of histone $\mathrm{H3}$ lysine residues in skeletal muscle}

Relative levels of methylated lysine residues on histone $\mathrm{H} 3$ were quantified in skeletal muscle assessing monomethylation of $\mathrm{K} 4(\mathrm{H} 3 \mathrm{~K} 4 \mathrm{me})$, trimethylation of $\mathrm{K} 9\left(\mathrm{H} 3 \mathrm{~K} 9 \mathrm{me}^{3}\right)$, monomethylation of $\mathrm{K} 27$ (H3K27me) and dimethylation of $\mathrm{K} 36\left(\mathrm{H} 3 \mathrm{~K} 36 \mathrm{me}^{2}\right)$ residues over the seven time points of the torpor/arousal cycle (Fig 3.4). Primary antibodies specific for histone $\mathrm{H} 3$ lysine residues that are methylated at the indicated lysine residue were used (Appendix B). Relative levels of $\mathrm{H} 3 \mathrm{~K} 4 \mathrm{me}$ did not change significantly during entrance, torpor, or EA although it should be noted that significant differences would be seen in the $\sim 4$ fold increases in EN and ET as compared to EC in a Student's t-test $(\mathrm{p}<0.05)$, but are confounded by wide variability of data in LA and IA time points. H3K4me levels rose strongly when squirrels aroused from torpor, being $11 \pm 1.8$ fold higher than EC values during LA (and at least 2.4 fold higher than all other time points), and then increasing further to $13 \pm 1.6$ fold over EC values during IA (and at least 2.9 fold higher than all other time points). Relative levels of $\mathrm{H} 3 \mathrm{~K} 9 \mathrm{me}^{3}$ showed significant increases in ET by $2.79 \pm 0.33$ fold over EC values. No change in $\mathrm{H} 3 \mathrm{~K} 9 \mathrm{me}^{3}$ levels was seen during LT and EA, but levels were decreased in both LA and IA, to $0.56 \pm 0.11$ fold and $0.72 \pm$ 0.09 fold, respectively, compared to EC values. Generally, H3K27me was not significantly changed from EC, except in ET when levels increased by $2.9 \pm 0.37$ fold over EC values (at least $2.2 \pm 0.28$ fold higher than all other time points). However, H3K27me levels fell in LT to one half of the ET maximum, and by EA decreased further to three-quarters of the ET maximum, before returning to control values in IA. Relative levels of $\mathrm{H} 3 \mathrm{~K} 36 \mathrm{me}^{2}$ did not change across the torpor/arousal cycle, with the exception of LA when levels rose at least $2.9 \pm 0.49$ fold over all other time points. 


\section{Analysis of the methylation status of HSP90 in skeletal muscle}

Relative levels of lysine methylation on HSP90 were also quantified in skeletal muscle

(Fig 3.5) using an antibody specific to methyllysine residues, and quantifying relative densities of a band at $90 \mathrm{kDa}$ that corresponds to the position of HSP90. Relative protein levels of HSP90, as compared to EC levels, were increased significantly in LT (1.7 \pm 0.05 fold higher), and decreased significantly in IA $(0.42 \pm 0.04$ fold decrease from EC; decreased $0.66 \pm 0.25$ fold from LT maximum values). Compared to EC levels of HSP90me, relative methylation content at 90kDa increased strongly in LT ( $3.5 \pm 0.20$ fold higher than EC levels), and remained elevated in arousal; EA levels of HSP90me were $1.8 \pm 0.01$ fold higher than EC levels although significantly decreased from LT maximum levels $(0.48 \pm 0.004$ fold $)$ whereas LA levels of HSP90me were 3.3 \pm 0.16 fold higher than EC levels. Relative levels of methylated HSP90 decreased during IA to a value not significantly different than the EC control values.

\section{Discussion}

A number of previous studies have highlighted the impacts that differential protein expression has, and their similarities in terms of maintaining muscle function and homeostasis in non-hibernators (Enwere et al., 2014; Kötter et al., 2014) and hibernator systems alike (Allan and Storey, 2012; Buck et al., 2002; Postnikova et al., 1999). The studied KMTs, SUV39H1, SMYD2, SET8, SET7/9, G9a, ASH2L and RBBP5, are known to be regulators of a number of cellular processes in non-hibernators, especially the control of transcriptional activity by their methyltransferase activity either on histone protein tails, or transcription factors (Biggar and Li, 2015; Zhang and Reinberg, 2001). Because skeletal muscle cells' activity is under extensive transcriptional and cellular regulation during hypometabolic states - as seen in hibernating mammals - the expression and function of transcriptional and cellular regulators - such as KMTs - are likely also tightly controlled in the transitions to and during torpid and aroused states. Since 
the context of hibernating skeletal muscle has never been explored in relation to the molecular mechanisms under the control of KMTs, the present results attempt to fill a gap in the research, and allows for speculation on their roles in the altered cellular environments that accompany mammalian hibernation.

It stands to reason that the differences in KMT enzyme abundance that are seen in skeletal muscle of the 13LGS (Figures 3.1 and 3.2) are likely key to regulating cellular processes during the torpor/arousal cycle (Srere et al., 1992; Storey, 2015). Similarly, differential KMT enzyme expression and abundance has already been shown to influence cellular activity in ways that are factor-specific, context-specific and dependant on the temporal presence of required complex members and/or substrate modifications, in non-hibernator systems (Aguilar-Arnal and Sassone-Corsi, 2013; Özdağ et al., 2006; Son et al., 2016). An overall pattern of KMT enzyme abundance did not emerge, likely the result of each studied factor being responsible for a variety of cellular processes at different times, some of which may interfere with or complement other studied factors. However, between EN and IA, five of the seven enzymes (SUV39H1, SMYD2, SET8, RBBP5 and ASH2L) reached minimal or near-minimal levels between the ET and LT time points compared to at least one preceding time point, and then protein amount increased in the subsequent time points, typically during both LA and IA (although only during LA in the case of SET8 and SET7/9). For example, both SMYD2 and ASH2L showed increases in protein abundance during ET, which was followed by a significant decrease in enzyme abundance during LT, and a recovery of protein levels across the arousal time points until IA. Given the physiological and phenotypic differences that characterize skeletal muscle during torpid and aroused states, it is possible that skeletal muscle KMT enzyme activity is actively involved in transitions into the torpid state (as evidenced by heightened protein levels during EN or ET and decreased during ET or LT), as well as intricately involved throughout the regulation of arousal (as evidenced by heightened protein levels during LA or IA). This rule though, does not hold true in all cases as protein amounts of SET7/9 are heightened throughout the torpor/arousal cycle 
except for minimum levels during the transition to arousal. SET7/9, whose enzymatic activity typically targets $\mathrm{H} 3 \mathrm{~K} 4$ leading to activation of transcription and inhibition of deactivating $\mathrm{H} 3 \mathrm{~K} 9$ methylation modifications, may not be involved in regulating the modification status of nuclear proteins; the transport of SET7/9 to the nucleus is under glycemic control, and as such, the enzyme acts as a sensor of hyperglycemia. When low levels of glucose are experienced - as in skeletal muscle cells during hibernation -nuclear localization of, and ultimately histone modifications by, SET7/9 are prevented (Keating and El-Osta, 2013a; Okabe et al., 2012). Of relevance then, are the dozens of cytoplasmic proteins, with an even higher number of relevant cellular processes, that may be uniquely targeted by sequence specificity for RPM by SET7/9 (Dhayalan et al., 2011; Zhang et al., 2016). Although the exact downstream effectors of SET7/9 activity are beyond the scope of this study, of particular relevance to hypometabolic skeletal muscle cells are important roles for SET7/9 in attenuating transcription via methylation of proteins involved in a variety of signaling cascades, including the NF- $\kappa \mathrm{B}$ (Li et al., 2008), hypoxia-inducible factor-1a (Kim et al., 2016), and estrogen receptor alpha signaling pathways (Subramanian et al., 2008; Zhou et al., 2009) to name a few. Differential amounts of SET7/9 during torpor may contribute to altered cytoplasmic transcription factor binding, localization or stability, and may even facilitate the expression of target genes by modulating the methylation status of transcription factors. Increased amounts of SET7/9 were indicated during arousalspecific increases in cellular glucose levels that promote the enzyme's nuclear translocation and histone methyltransferase activity. These increases in protein amount also correlate with increased NF- $\mathrm{\kappa B}$ and hypoxia-inducible factor-1a target gene expression in skeletal muscles of hibernating ground squirrels undergoing arousal, and allude to these pathway's dependence on other mechanisms for torpor-specific increases in target gene transcription (Allan and Storey, 2012; Morin and Storey, 2005; Zhu et al., 2005). The other enzyme that does not follow the ENIA pattern seen in the other five enzymes is G9a, which shows strong enhancements in protein levels during the transitory periods (ET and EA) of the torpor/arousal cycle, although not during 
EN. G9a is widely regarded to act as a repressor of transcription through the addition of inhibitory histone $\mathrm{H} 3$ modifications - specifically mono- and dimethylation activity at K9 $\left(\mathrm{H} 3 \mathrm{~K} 9 \mathrm{me} / \mathrm{me}^{2}\right)$ and weaker activity on $\mathrm{K} 27\left(\mathrm{H} 3 \mathrm{~K} 27 \mathrm{me} / \mathrm{me}^{2}\right)$ although only dimethylation of the latter lysine residue is repressive to transcription (Shankar et al., 2013; Tachibana et al., 2001; Wu et al., 2011). G9a histone methylation activity at H3K9 and H3K27 in ET may accommodate the global attenuation of transcriptional activity that occurs during hypometabolism, although this mechanism is unexplored within hibernators. Strong increases in G9a levels during EA on the other hand, may serve important roles in attenuating transcription factor binding, or recruiting transcriptional co-repressors, for example (Karpovich et al., 2009). Similarly, G9a may act in concert with the transcriptional repressors histone deacetylase 1 (HDAC1) or DNA methyltransferase 1 (Chin et al., 2007; Rathert et al., 2008), given a lack of increased methylation at the aforementioned histone lysine residues, as well as increases in the amount and activity of HDAC enzymes in the skeletal muscle of hibernating 13LGS (Morin and Storey, 2006).

Decreases in abundance of the other five KMT enzymes, SUV39H1, SMYD2, SET8, ASH2L and RBBP5, were observed during either ET or LT compared to prior or subsequent time points (as mentioned above). Increased amounts of SUV39H1 during torpor would at first be expected, due to its trimethylation activity at the transcriptional-repressor H3K9 (Peters et al., 2002); however, the opposite appears to be true as SUV39H1 abundance is significantly decreased during ET compared to arousal (Figure 3.1). An interaction between the already discussed KMT enzyme SET7/9, and SUV39H1 may occur and is made more likely given increases in SET7/9 abundance during torpor and arousal, and the inhibitory methylation of SUV39H1 by SET7/9 (Wang et al., 2013). Given that SUV39H1 is largely involved in the repression of transcriptional activation via chromatin re-organization and the recruitment of heterochromatin 1 (HP1) and methyl-CPG binding domains, both being associated with transcription repression rather than activation, increased SUV39H1 abundance during arousal may be tied to roles in maintaining genomic organization and stability (Fujita et al., 2003; 
Jenuwein, 2006; Rea et al., 2000; Sidler et al., 2014). Maintenance of genomic organization, DNA damage response targeting and cell cycle control are also regulated by SET8, whose protein levels do not change across the torpor/arousal cycle, but show slight non-significant increases in protein amount during arousal. Since SET8 is involved in transcriptional activation via methylation of histone $\mathrm{H} 4$ and the recruitment of Condensin II or p53 binding partner-1 to chromatin (Beck et al., 2012), the enzyme's role as a docking site mediator may be important for chromosome condensation as well as DNA damage responses (which are targeted to damaged DNA in part by dimethylated H4K20, recognized by p53 binding partner-1) during torpor (Beck et al., 2012; Botuyan et al., 2006). Dysregulated H4K20 methylation has been linked with aberrant chromatin organization and decreased DNA damage response efficacy (Schotta et al., 2008). Given SET8's essential roles in cell cycle and genomic control, slight increases during arousal may therefore be caused by the protein's turnover between torpor/arousal cycles.

Finally, the three KMT enzymes, SMYD2, ASH2L and RBBP5 showed very similar patterns of protein abundance (Figures 3.1 and 3.2). Protein levels increased during EN and ET, and then in all three cases fell significantly during LT before rebounding by IA, and in the case of ASH2L and RBBP5, heightened significantly above EC levels as well. Since ASH2L and RBBP5 work in complex to target H3K4 for mono-, di-, or trimethylation (Cao et al., 2010; Patel et al., 2009), their similarities in abundance are to be expected. Similarly, SMYD2 is known to have strong methyltransferase specificity for $\mathrm{H} 3 \mathrm{~K} 4 \mathrm{me}$ and $\mathrm{H} 3 \mathrm{~K} 36 \mathrm{me}^{2}$, as well as roles in modifying other non-histone proteins including HSP90 and p53 (Abu-Farha et al., 2008; Huang et al., 2006; Olsen et al., 2016). Methylation of H3K4 has been strongly linked to the activation of transcription, with increasing methyl-marks increasing the degree of activation (Rosenfeld et al., 2009; Wozniak and Strahl, 2014). Increased methylation activity at H3K4 during torpor is further suggested by significantly increased methyltransferase activity during EN, and increased H3K4 methylation during EN and ET (Figures 3.3 and 3.4, further discussed in the following paragraph) Since transcription is a downregulated process during hibernation, it could be that the 
methylation of H3K4 in skeletal muscle is targeted at hibernation-specific genes, wherein methylation of H3K4 by SET7/9, ASH2L, RBBP5 or any number of H3K4-specific KMT enzymes localize transcriptional activity to the required chromosomal regions (Liang et al., 2004; Ruthenburg et al., 2007; Zhang and Reinberg, 2001). However, current results simply correlate increased $\mathrm{H} 3 \mathrm{~K} 4$ methylation activity with certain time points within torpor/arousal cycles and do not allude to the tissues dependence on the modification for changes in transcriptional activity.

As mentioned, the methyltransferase activity in skeletal muscle was also investigated, and it was found that activity at the activating mark $\mathrm{H} 3 \mathrm{~K} 4$ and the inhibitory $\mathrm{H} 3 \mathrm{~K} 9$ mark showed differential regulation across the torpor/arousal cycle, while activity levels of the inhibitory H3K27 mark showed no significant differences (Figure 3.3). During hibernation, it might be expected that activity levels of the inhibitory marks would increase, however, this is not the case since H3K9 decreases between EN and LT, while H3K27 methyltransferase activity levels remained the same as EC. Taken together with the relative histone methylation measurements on corresponding histone-lysine residues across torpor and arousal (Figure 3.4), it would appear as if heightened methylation activity at $\mathrm{H} 3 \mathrm{~K} 9$ and $\mathrm{H} 3 \mathrm{~K} 27$ is restricted to ET. An interesting pattern in relative histone methylation levels emerge, wherein levels of $\mathrm{H} 3 \mathrm{~K} 4 \mathrm{me}$ are increased in both EN and ET compared to control animals (significant using student's t-test), but drop off during LT at the same time as levels of $\mathrm{H} 3 \mathrm{~K} 9 \mathrm{me}^{3}$ are increased, beginning in ET. Given that methylation activity at $\mathrm{H} 3 \mathrm{~K} 4$ in LT is reduced compared to activity during EN, as are levels of monomethylation on the same lysine residue in LT compared to EN and ET, it may be that increases in $\mathrm{H} 3 \mathrm{~K} 9 \mathrm{me}^{3}$ during ET are inhibiting methylation on $\mathrm{H} 3 \mathrm{~K} 4$. Differential levels of $\mathrm{H} 3 \mathrm{~K} 4 \mathrm{me}$ and $\mathrm{H} 3 \mathrm{~K} 9 \mathrm{me}^{3}$ during torpor may be influenced by histone demethylation, and an inhibitory crosstalk between RPM of the opposing lysine residues (Shi et al., 2011; Yamane et al., 2006). In non-hibernators, increases in $\mathrm{H} 3 \mathrm{~K} 4 \mathrm{me}$ contribute to localizing transcriptional activity to essential genes, and the use of this mechanism to hibernators may be evident. Interestingly, an increases in methylation of $\mathrm{H} 3 \mathrm{~K} 4$ correlates with increases in the number of 
skeletal muscle satellite cells present in ET. Satellite cells in skeletal muscle are involved in the growth and repair of muscle, and torpor-specific increases in the number of satellite cells are presumed to influence the regeneration of muscle tissue following injury in hibernation as well as regulation of the extracellular environment, although satellite cell levels are brought back to control levels in LT (Andres-Mateos et al., 2012; Brooks et al., 2015). Increased numbers of skeletal muscle satellite cells were also associated with control levels of myogenic factor 5 which is under the control of the transcription factor paired box 7 (Pax-7). Previous research has shown that during satellite cell quiescence, Pax-7 binds with the ASH2L-MLL (mixed-lineage-leukemia) complex, of which RBBP5 is also a member, to methylate H3K4 around the myogenic factor 5 gene as well as other Pax-7 target genes (McKinnell et al., 2008; Montarras et al., 2013). The influence of transcriptionally-repressive methylation on K9 and transcriptional-activating methylation on $\mathrm{K} 27$ of histone 3, also in ET, may or may not be relevant to the generation of skeletal satellite cells; strong increases in $\mathrm{H} 3 \mathrm{~K} 9 \mathrm{me}^{3}$ and $\mathrm{H} 3 \mathrm{~K} 27 \mathrm{me}$ may limit and localize the transcriptional activation present in, and in turn, the functional activity of satellite cells, as well as prevent satellite cells from committing to myogenic differentiation (Chen and Dent, 2014; Liu et al., 2013) - a finding which would agree with findings showing the number of myonuclei present within skeletal muscle fibers is constant across the torpor/arousal cycle (Brooks et al., 2015; Malatesta et al., 2009). The extent to which histone methyllysine modifications play a role in the maintenance and proliferative ability of skeletal muscle satellite cells during mammalian torpor has yet to be explored.

Lastly, significant increases in methylation modifications associated with active transcription (i.e., $\mathrm{H} 3 \mathrm{~K} 4 \mathrm{me}$ and $\mathrm{H} 3 \mathrm{~K} 36 \mathrm{me}^{2}$ ) during arousal, may transition the genome from an environment promoting transcriptional suppression to one that promotes the turnover of proteins essential for subsequent bouts of torpor. Monomethylation of H3K4 specifically, as opposed to di- or trimethyl- modifications of the same residue, are associated with demarcating areas of transcriptional activation. Within active genes, increased degrees of H3K4 as well as H3K36 
methylation are found (i.e., $\mathrm{H} 3 \mathrm{~K} 4 \mathrm{me}^{2} / \mathrm{me}^{3}$ and $\mathrm{H} 3 \mathrm{~K} 36 \mathrm{me}^{2} / \mathrm{me}^{3}$ ) (Huang et al., 2011; Li et al., 2007), however monomethylation of $\mathrm{H} 3 \mathrm{~K} 4$, may also serve as a binding site for transcriptional repressors when found within intragenic regions of chromatin in the absence of higher degree methylation modifications (Cheng et al., 2014). On the other hand, findings which show that enrichment patterns of $\mathrm{H} 3 \mathrm{~K} 4$ me modifications are congruent with enrichment patterns of $\mathrm{H} 3 \mathrm{~K} 4 \mathrm{me}^{2} / \mathrm{me}^{3}$ in promoting gene activivity across broad regions of the genome (Rosenfeld et al., 2009), it is most likely that during arousal, increased levels of transcriptionally-permissive methylation modifications are allowing for the regeneration of necessary proteins for example, while temporally restricting their synthesis to periods of arousal (Brooks et al., 2011; Dang et al., 2016; Fedorov et al., 2014). Similarly, research has shown that increased dimethylation of H3K36 specifically, is required for preventing aberrant polymerase II activity through histone deacetylase recruitment to the genome in the wake of transcription (Hsia et al., 2010; Li et al., 2009), and the recruitment of early response proteins during DNA-damage responses (Fnu et al., 2011), both of which would allude to a transcriptionally active phenotype characterizing the arousal stage of mammalian hibernation.

Of course, histones are not the only target of KMT enzymes, as an incredibly diverse range of proteins may be influenced by RPM. Over-expression of chaperone proteins, especially HSP90, is a hallmark of findings in tissues experiencing environmental temperature changes (Feder and Hofmann, 1999; Wu et al., 2015; Xu et al., 2013). Within skeletal muscle, the action of SMYD2 on the chaperone protein HSP90 at K616 (giving rise to HSP90K616me), is crucial for the formation of chaperone complexes that protect large sarcomeric proteins, whereby deficiencies impair sarcomere stability and muscle function, especially in the initiation of shivering thermogenesis (Donlin et al., 2012; Du et al., 2014; Taylor-Burt et al., 2015). Surprisingly, protein levels of HSP90 were seen to fall to control levels in EA (Figure 3.5), at the same time that reductions in RPM of HSP90 were also seen, following significant upregulations of both in LT. HSP90me detection was significantly upregulated in EA compared to EC, although 
given the reduction in levels of HSP90me seen in EA to one-half of protein abundance seen in LT, and only minor reductions in total HSP90 protein amount in EA compared to LT, it is likely that cells are actively demethylating HSP90 in EA. On the other hand, significant increases in methylated HSP90 in LA, and constant levels of HSP90 compared to EA, point to increased KMT activity; increased methylation of HSP90 in LA is especially relevant since at this point in the torpor/arousal cycle skeletal muscle tissue is actively regulating shivering thermogenesis activity in order to aid in the animal's rewarming (Drew et al., 2007; Haman, 2006). These findings make it apparent that skeletal muscle cells are relying on the actions of RPM on HSP90 to enhance protection of the sarcomere during times of increased shivering thermogenesis (i.e., arousal). Furthermore, this finding also shows that RPM may be serving to increase cytoprotective mechanisms in times of altered cellular environments, and that these changes are directly modulated by differential regulation of KMT enzymes. Previous studies have highlighted a role for methylation of HSP90 during shivering thermogenesis, although the current finding seems to be the first to show skeletal muscle in mammalian hibernators may be relying on a very similar mechanism under the control of RPM (Hamamoto et al., 2015b; Khurana and Bhattacharyya, 2015; Voelkel et al., 2013).

In light of these intriguing insights, RPM by the evolutionarily conserved KMT enzymes may play an integral role in a number of cellular pathways within skeletal muscle in a mammalian hibernator, however the present research has only just begun to characterize the KMT enzymes and their actions in this context. Similarly, much work remains to be done to fully elucidate the possible roles that these enzymes play in regulating the metabolic and protective pathways that are central to muscle tissue's survival. It appears that the protein amounts of KMTs may indeed be characteristic of this natural process, and is likely another example of the conserved function of RPM in the universal regulation of metabolic homeostasis and cellular protective responses. Perhaps more importantly, this also represents the first studies investigating regulation over RPM in the context of hibernation. Findings that show differential amounts of KMT enzyme levels, in 
many cases elevated during either torpor or arousal, as well as modulation of KMT enzymatic activity and differential methylation of downstream targets, the data suggest that these enzymes are expressed and active in the regulation of skeletal muscle responses to hibernation. It appears likely that KMT enzyme activity is especially involved in the arousal from torpor, given interesting correlations in the protein levels of SUV39H1, SMYD2, SET8, ASH2L and RBBP5, and the methylation activity on $\mathrm{H} 3 \mathrm{~K} 4$ and $\mathrm{H} 3 \mathrm{~K} 9$ as well as their methylation status. Research by our lab has also shown that differential abundance of the sirtuin- 6 deacetylase enzyme may play similar roles in protecting skeletal muscle cells during arousal from hibernation (Rouble and Storey, 2015), whereby sirtuin-6 may alter DNA-damage repair proteins, and increase their activity during times of increased oxidative stress. The influence of epigenetic and non-epigenetic mechanisms in the control over genome regulation means that these enzymes may be involved in modifying the activities of a vast number of cellular pathways during mammalian hibernation. Furthermore, given that many chromatin modifier enzymes also modify non-histone proteins, research into the regulatory mechanisms of epigenetic enzymes is of importance to not just cellular responses to altered environments, but in all aspects of life science. 

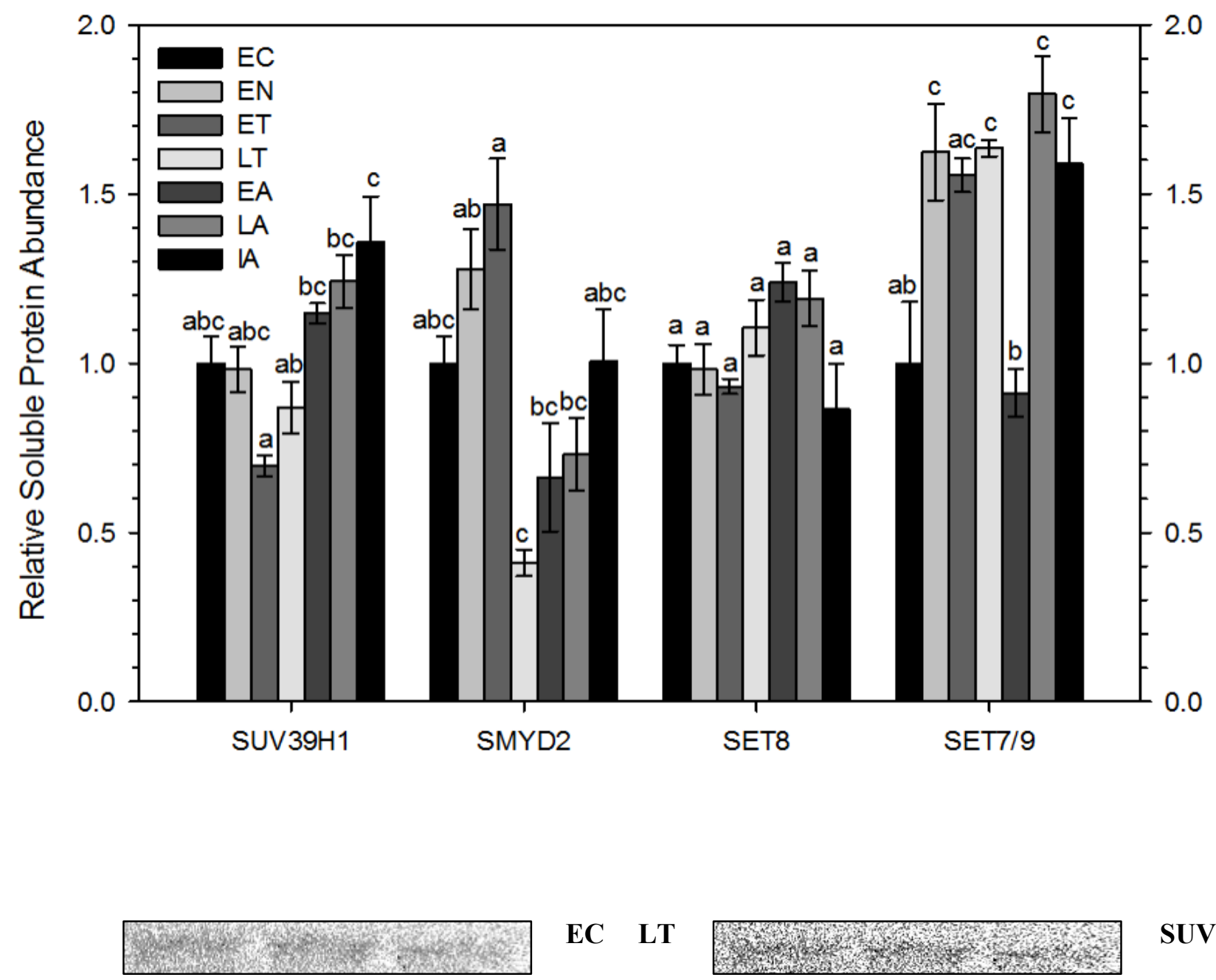

EC LT
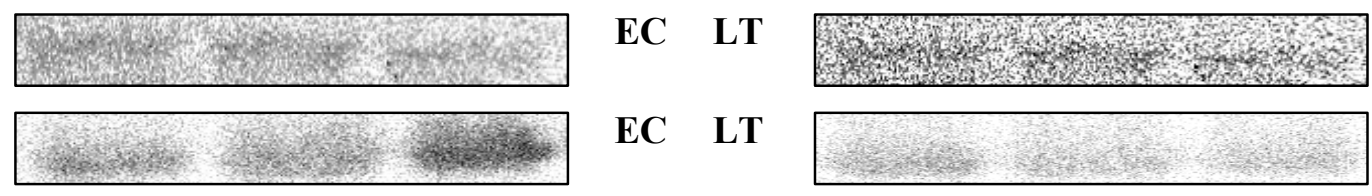

EC LT
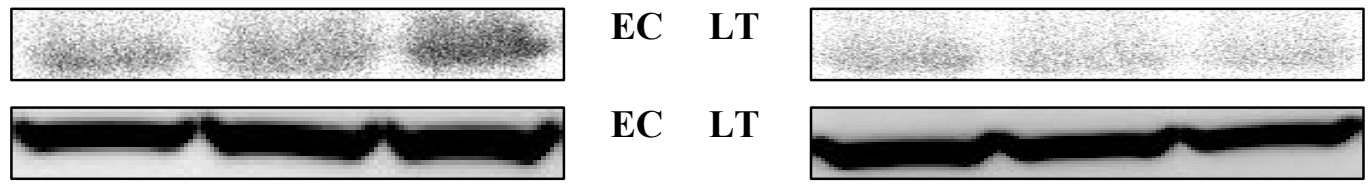

EC LT

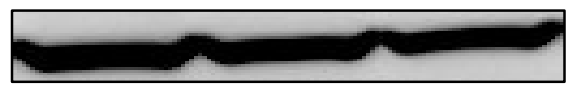

SET8

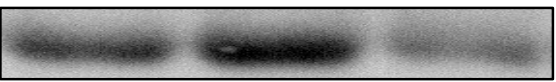

EC EN

SMYD2

SET7/9

Figure 3.1: Relative protein abundance of SUV39H1, SMYD2, SET8 and SET7/9 in skeletal muscle of I. tridecemlineatus over the torpor/arousal cycle along with three representative immunoblot protein bands for selected sampling points (labelled between the blots). Sampling points are: euthermic in the cold room (EC), entrance into torpor (EN), early torpor (ET), late torpor (LT), early arousal (EA), late arousal (LA) and interbout arousal (IA); See Chapter 2: Materials and Methods for more extensive definitions. Histograms show mean standardized band densities ( \pm SEM, $n=4$ independent trials on tissue from different animals). Protein bands were standardized against the summed intensity of a group of Coomassie-stained protein bands from the same sample lane. Data were analyzed using a one-way ANOVA with a post hoc Tukey test. Different letters denote values that are significantly different from each other $(p<0.05)$. 

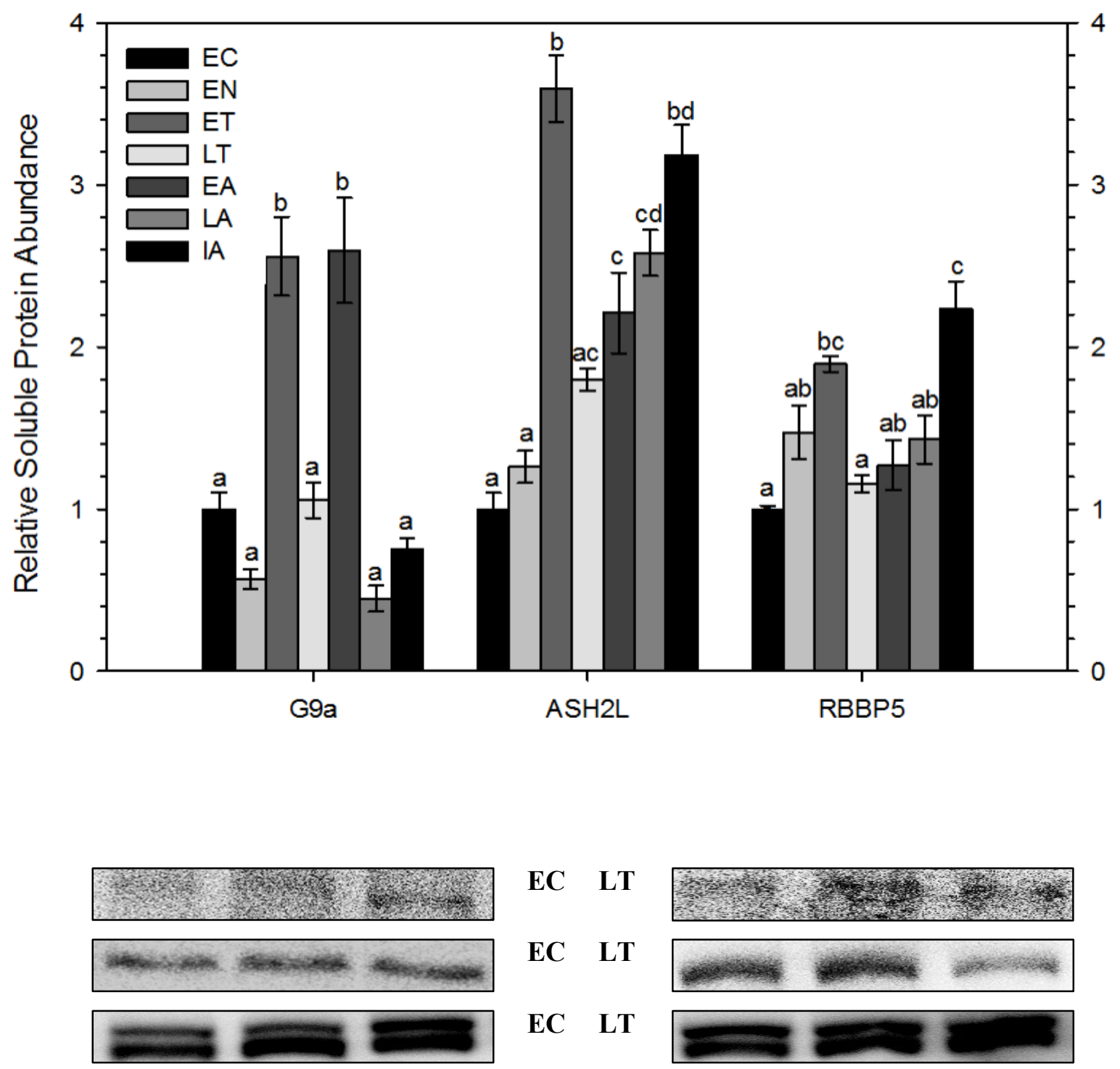

G9a

ASH2L

RBBP5

Figure 3.2: Relative protein abundance of G9a, ASH2L and RBBP5 in skeletal muscle of $I$. tridecemlineatus over the torpor/arousal cycle along with three representative immunoblot protein bands for EC and LT sampling points (labelled between the blots). All other information is as in Fig. 3.1. 


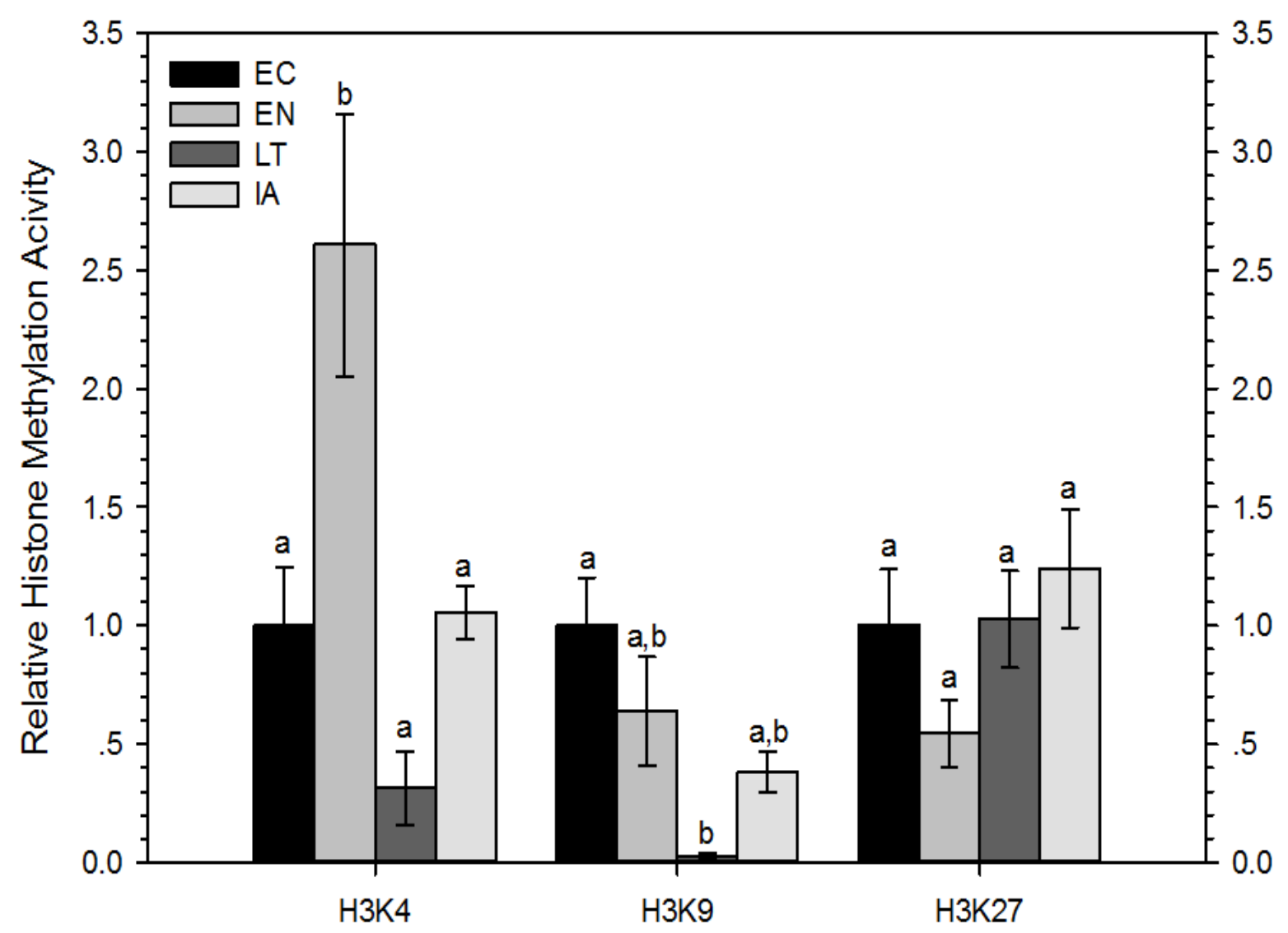

Figure 3.3: Relative histone methyltransferase activity at $\mathrm{H} 3 \mathrm{~K} 4, \mathrm{H} 3 \mathrm{~K} 9$ and $\mathrm{H} 3 \mathrm{~K} 27$ in skeletal muscle total soluble protein extracts from I. tridecmlineatus comparing euthermic in the cold room (EC) to entry to torpor (EN), late torpor (LT) and interbout arousal (IA) time points of the torpor/arousal cycle. Histograms show mean amount of methyllysine produced per hour per weight protein added $(\mathrm{ng} / \mathrm{h} / \mathrm{mg}) \pm \mathrm{SEM}, \mathrm{n}=3-4$ independent assays on tissue from different animals. Histone methyltransferase activity at $\mathrm{H} 3 \mathrm{~K} 4, \mathrm{H} 3 \mathrm{~K} 9$ and $\mathrm{H} 3 \mathrm{~K} 27$ was found to be 2.47 $\pm 0.25 \mathrm{ng} / \mathrm{h} / \mathrm{mg}, 4.68 \pm 0.31 \mathrm{ng} / \mathrm{h} / \mathrm{mg}$ and $0.43 \pm 0.21 \mathrm{ng} / \mathrm{h} / \mathrm{mg}$, respectively in control animals, with all other time points shown relative to control activity. Data were analyzed using a one-way ANOVA with a post hoc Tukey test. Different letters denote values that are significantly different from each other $(\mathrm{p}<0.05)$. 


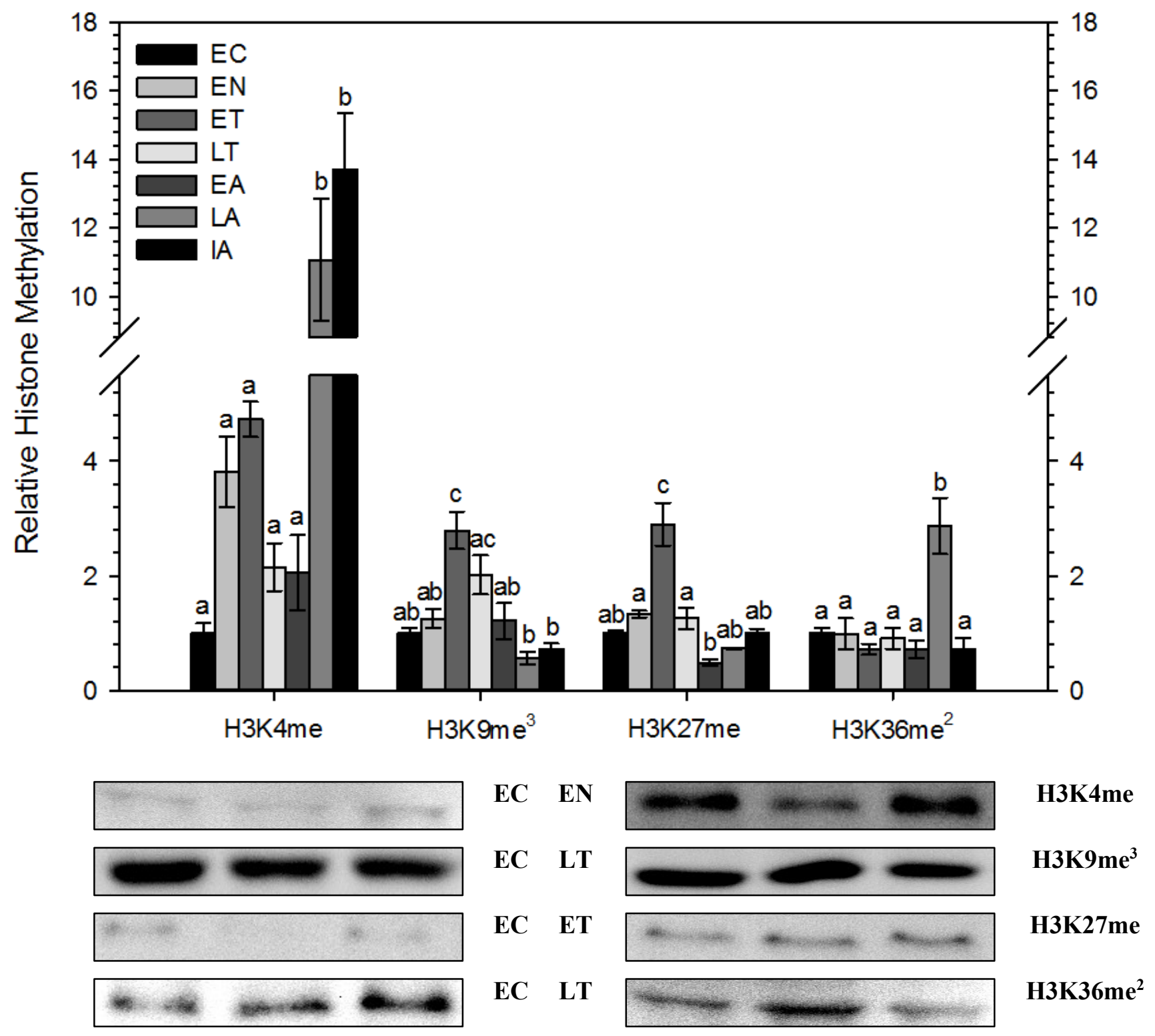

Figure 3.4: Relative amounts of histone $\mathrm{H} 3$ monomethylated at lysine 4 (H3K4me), trimethylated at $\mathrm{K} 9\left(\mathrm{H} 3 \mathrm{~K} 9 \mathrm{me}^{3}\right)$, monomethylated at $\mathrm{K} 27$ (H3K27me), or dimethylated at K36 $\left(\mathrm{H} 3 \mathrm{~K} 36 \mathrm{me}^{2}\right)$ in skeletal muscle of I. tridecemlineatus over the torpor/arousal cycle along with three representative immunoblot protein bands for EC and LT sampling points (labelled between the blots). All other information is as in Fig. 3.1. 

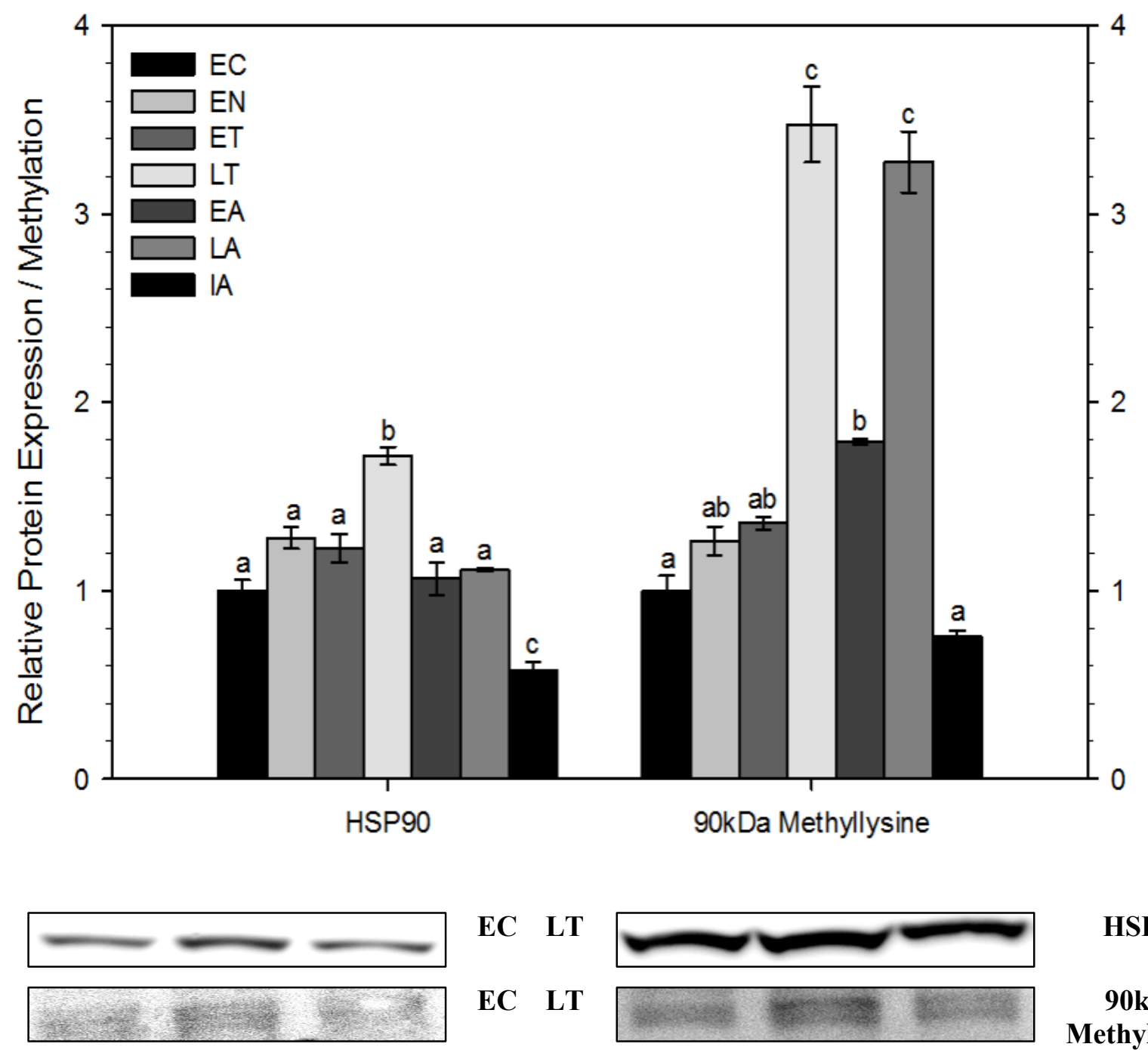

HSP90

EC LT

90kDa

Methyllysine

Figure 3.5: Relative protein abundance of HSP90 and methyllysine (quantified at 90kDa) in skeletal muscle of I. tridecemlineatus over the torpor/arousal cycle along with three representative immunoblot protein bands for EC and LT sampling points (labelled between the blots). All other information is as in Fig. 3.1. 


\section{Chapter 4}

Analysis of Histone Methyltransferase Enzyme Abundance and their Downstream Targets in Liver during Mammalian Hibernation 


\section{Introduction}

The liver is an important organ whose function is to control an array of metabolic processes that affect the entire body, including regulation of glycogen stores, lipid synthesis, hormone production, detoxification of metabolites and xenobiotics, and the synthesis of many proteins that are exported into the blood. During mammalian hibernation, major changes in a mammal's metabolic strategies are involved, requiring a great deal of change to the activity and use of certain metabolic pathways under regulation by the liver. The influence of changes in the PTM status of liver proteins has already been shown to affect metabolic activity during hibernation (Rouble and Storey, 2015; Storey, 1987; 1997), providing a rationale for the characterization of the enzymes in control of RPM in the same organ.

Furthermore, in order to regulate the changes in liver physiology, differences in the expression levels of cytoprotective proteins have been observed as necessary during mammalian hibernation (Brown et al., 2012; Rouble et al., 2013; Srere et al., 1992; Wu and Storey, 2012b). For example, the mitochondrial chaperone protein, glucose-regulated protein 75 (grp-75), is required for the efficient import of proteins to the mitochondria and refolding of proteins during oxidative stress responses (E et al., 2013; Wadhwa et al., 2002); protein levels of grp-75 were significantly upregulated in the liver during torpor compared to abundance in active 13LGSs. This pattern was reasoned to facilitate pro-survival pathways in the face of increased protein synthesis and mitochondrial activity during arousals, both of which are likely to generate oxidative stress (Carey et al., 1999), as evidenced by correlated increases in anti-oxidant enzymes (Biggar et al., 2015; Ni and Storey, 2010; Rouble and Storey, 2015; Rouble et al., 2013; Vucetic et al., 2013). As described in Chapter 1, the RPM of the p53 transcription factor has multiple effects on transcriptional activity depending on where methyl-modifications are placed, as well as the number of methyl-modifications placed on a single lysine residue (Scoumanne and Chen, 2008). Within the liver, p53 localizes to the nucleus and binds DNA during torpor in goldenmantled ground squirrels, but transcriptional activity of p53 was not observed by analysis of gene 
transcript levels of some of its downstream targets, likely due to an arrest of transcriptional elongation (Pan et al., 2014). The methylation status of $\mathrm{p} 53$ does play a role in the binding of DNA (Scoumanne and Chen, 2008; West and Gozani, 2011), and it is therefore likely that during mammalian hibernation p53 would show methyl-modifications which correlate with DNA binding.

Due to the influence of histone methylation over transcriptional activation and repression, it is likely that RPM of histone proteins plays a role in regulating the switch from a summer preference for carbohydrate oxidation to the more versatile lipid oxidation in winter.

In order to slow the rate of carbohydrate metabolism, the 13LGS intricately regulates not only the expression, but also the activity of key glycolytic enzymes including pyruvate kinase and pyruvate dehydrogenase, as well as enzymes involved in the malate:aspartate shuttle, which is required for the efficient import of glycolytic electron equivalents to the mitochondria; during torpor decreases in the protein levels and activity of glycolytic enzymes decrease glycolytic pathway activity within the liver by about 50\% (Brooks and Storey, 1992; Epperson et al., 2010a; Khu et al., 2014). A related downregulation of enzymes involved in gluconeogenesis or amino acid catabolism was also shown to benefit the animal through the avoidance of unnecessary fuelstorage and protein turnover, seen in decreased levels of glycogen and protein degradation during torpor (Galster and Morrison, 1975; Gehnrich and Aprille, 1988; Klain and Whitten, 1968; Whitten and Klain, 1968). Lastly, an upregulation of peroxisomal and lipolytic enzymes as well as an associated increase in lipid-catabolism activity is characteristic of mammalian hibernation and allows the animal to preserve ATP that would otherwise be used in less-efficient glycolytic processes (Hindle et al., 2014; 2011). The multitude of changes in gene expression and enzymatic activity that occur in the liver rely on the work of several endocrine regulators and therefore has parallels to the human condition, type 2 diabetes (Carey et al., 2003; Klug and Brigham, 2015; Wu et al., 2013). Interestingly, the methylation status of histone $\mathrm{H} 3$ was shown to be effected in a mouse model of type 2 diabetes, wherein levels of $\mathrm{H} 3 \mathrm{~K} 4 \mathrm{me}$ and $\mathrm{H} 3 \mathrm{~K} 9 \mathrm{me}^{2}$ were increased and 
this effect could be rescued following treatment with an insulin-secretion stimulator (Tu et al., 2015). It has not currently been shown whether or not this shift requires the influence of differential PTMs on histone proteins, although given the ability of histone modifications to discriminate and regulate the abundance of specific proteins over time-scales of hours to days, it stands to reason that this mechanism may also regulate physiological changes taking place within the liver (Keating and El-Osta, 2015; Tian and Xu, 2015).

Within the liver, the actions and roles of RPP and RPA have been partially elucidated. For instance, phosphorylation of a variety of proteins may influence the stimulation or inhibition of metabolic pathways through their actions on metabolic enzymes, ribosomal activity via modification of the ribosome, transcription factor-genome interactions or even the metabolic fuel concentration within cells (Rubin and Rosen, 1975; Villén et al., 2007; Zhao et al., 2010). In addition, RPP and RPA may both target histone proteins, which may accentuate the effects of protein PTMs by altering the amounts of either essential or unnecessary proteins (Tian and $\mathrm{Xu}$, 2015). Similar mechanisms for altering cellular activities and functions may be seen in the actions of RPM on lysine residues; lysine methylation may also occur on either histone or non-histone proteins and may also affect any number of cellular pathways or mechanisms at any one time through differential modification of target proteins. As previously mentioned, diseases of the liver have already been shown to influence the methylation status of histone $\mathrm{H} 3$, as have changes in cellular environments and homeostatic mechanisms in other organs and tissues, which shows that in times of cellular stress, changes in the modification status of proteins alter the cell's survival and overall activity (Martin and Zhang, 2005; Miao et al., 2014; Wang et al., 2014).

The present chapter explores the role of RPM in regulating physiological changes that are required by liver cells during mammalian hibernation, in the form of differential methylation of proteins or enzymes that alter cytoprotective mechanisms or metabolic strategies. In order to reach this goal, the KMT enzymes, , SET and MYND domain-containing protein 2 (SMYD2), SET domain-containing protein 8 (SET8), SET domain-containing protein 7/9 (SET7/9), 
Retinoblastoma-binding protein 5 (RBBP5), G9a Histone Methyltransferase (G9a), and Set1/Ash2 histone methyltransferase complex subunit ASH2 (ASH2L) were characterized across the torpor/arousal cycle in liver of 13LGS. Relative enzymatic activities on histone H3 lysine residues were also characterized, specifically at lysine-4 (H3K4), lysine-9 (H3K9) and lysine-27 (H3K27), as well as the monomethylation of $\mathrm{H} 3 \mathrm{~K} 4$ (H3K4me), trimethylation of H3K9 $\left(\mathrm{H} 3 \mathrm{~K} 9 \mathrm{me}^{3}\right)$, and dimethylation of lysine-36 $\left(\mathrm{H} 3 \mathrm{~K} 36 \mathrm{me}^{2}\right)$. Lastly, changes in the methylation status of Cellular tumor antigen p53 (p53) were evaluated, p53 being a downstream target of SMYD2 that methylates K370 (p53K370me²), and of SET7/9 that methylates K372 (p53K372me). These studies assessed the correlation of modifications on the important p53 transcription factor with changes in the abundance of KMT enzymes during mammalian hibernation. Results shows that KMT enzymes are differentially expressed during torpor and arousal in the liver of the 13LGS and point to their likely influence over cellular regulation mechanisms.

\section{Materials and Methods}

\section{Animal experiments and total soluble protein extractions}

Animal experiments and total soluble protein extractions were conducted as described in

\section{Chapter 2.}

\section{Western blotting}

Western blotting was performed as described in Chapter 2. Protein levels of the studied KMTs (SMYD2, SET8, SET7/9, G9a, ASH2L and RBBP5) and the downstream targets, (H3K4me, H3K9me 3 , H3K36me 2 , p53K370me 2 , p53K372me) were analyzed in total soluble protein extracts of I. tridecemlineatus liver. Antibodies used were: anti-SMYD2, anti-SET8, antiSET7/9, anti-G9a, anti-ASH2L, anti-RBBP5, anti-H3(K4me), anti-H3(K27me), antiH3(K36me 2), anti-p53(dimethyl-K370), anti-p53(monomethyl-K372) (see Appendix B for full 
details on each antibody). For a detailed list of the experimental conditions used for each protein target please refer to Appendix D.

\section{Total histone methyltransferase activity assay}

Total histone methyltransferase activity specific to H3K4, H3K9 and H3K27 residues were assayed in total soluble protein extracts from liver using the EpiQuick Histone Methyltransferase Activity/Inhibition Assay Kit from Epigentek (Farmingdale, NY), as described

\section{in Chapter 2.}

\section{Quantification and Statistics}

Quantification and statistics were carried out as described in Chapter 2.

\section{Results}

\section{Analysis of liver KMT protein levels across the torpor/arousal cycle}

The relative protein levels of SMYD2, SET8, SET7/9, RBBP5, ASH2L and G9a in liver were compared across the seven time points of the torpor/arousal cycle. Antibodies for each of the proteins (Appendix A) reacted with the appropriate number of bands (dependent on the presence of isozymes) at the expected molecular mass, as shown by representative immunoblot bands in Figure 4.1.

Data for SMYD2, SET8, SET7/9 and RBBP5 are displayed in Figure 4.2. Relative protein levels of SMYD2 decreased during the initial torpor periods, EN, ET and LT to $-0.55 \pm$ 0.05 fold, $-0.58 \pm 0.06$ fold, and $-0.64 \pm 0.04$ of EC levels, respectively. Protein levels of SMYD2 then began to rise during EA, and returned to values not significantly different from control during LA and IA. As for relative protein levels of SET8, reduced abundance was seen in LT, where protein levels decreased to less than one-half of EC levels. However, protein levels rebounded and then surpassed EC levels so that by LA, SET8 abundance was $1.5 \pm 0.11$ fold higher than EC levels, and $2.4 \pm 0.18$ fold higher than the LT minimum level. By IA, abundance 
of SET8 had decreased back to control values. The relative protein levels of SET7/9 increased during EN, $1.5 \pm 0.10$ fold higher than the EC levels of protein amount, and remained elevated during ET (1.4 \pm 0.09 fold higher than EC) and LT (not significantly different from EC). Relative levels of SET7/9 fell during EA, but not significantly compared to EC levels, although significantly lower compared to LT, ET and the EN maximum (-0.56 \pm 0.06 fold of EN values). IA levels of SET7/9 were $1.4 \pm 0.05$ fold higher than EC levels. Relative levels of RBBP5 were strongly increased in EN to $1.7 \pm 0.10$ fold higher than EC levels, and then fell during ET and LT back to control levels. Abundance of RBBP5 remained at about EC levels through ET and LT but then fell in EA to a value that was $-0.61 \pm 0.04$ fold of EC levels. However, RBBP5 amount rose again in LA ( $1.6 \pm 0.02$ fold higher than EA) but then fell again to $-0.54 \pm 0.07$ fold of EC levels during IA.

Data for ASH2L and G9a are displayed in Figure 4.3. The relative protein levels of ASH2L increased during EN to $2.4 \pm 0.10$ fold higher than EC levels, and remained significantly higher than EC over the rest of the torpor/arousal cycle (all values greater than $1.6 \pm 0.04$ fold higher than EC). Relative protein levels of G9a increased during EN to $3.1 \pm 0.01$ fold higher than EC levels, before falling to control levels during ET and then falling lower during LT and EA. Protein levels of G9a rose again during LA to $3.1 \pm 0.47$ fold higher than EC levels but then decreased aging during IA.

\section{Assay of total histone methyltransferase activity in liver}

Total histone methyltransferase activity was quantified in total soluble protein extracts during the EC, EN, LT and IA periods of the torpor/arousal cycle in liver. Assays specific to histone-lysine residues were selected to assay methyltransferase activity at $\mathrm{H} 3 \mathrm{~K} 4, \mathrm{H} 3 \mathrm{~K} 9$ and H3K27 (Fig 4.3). Comparing relative methyltransferase activity at H3K4, activity was significantly reduced during IA to $0.43 \pm 0.01$ fold as compared with activity during EC but not at the other two time points. Relative methyltransferase activity at H3K9 showed no significant differences from EC levels, although a decrease in activity was seen between EN and IA, where 
methyltransferase activity in IA samples was decreased $-0.47 \pm 0.10$ fold of the maximum EN levels. Lastly, relative methyltransferase activity at H3K27 during IA was increased by $2.5 \pm 0.51$ fold over EC samples, and by $2.1 \pm 0.43$ fold compared with LT.

\section{Analysis of the methylation status of histone $\mathrm{H3}$ lysine residues in liver}

Relative levels lysine residues on histone $\mathrm{H} 3$ methylated at $\mathrm{K} 4$ (H3K4me), trimethylated at $\mathrm{K} 9\left(\mathrm{H} 3 \mathrm{~K} 9 \mathrm{me}^{3}\right)$ or dimethylated at $\mathrm{K} 36\left(\mathrm{H} 3 \mathrm{~K} 36 \mathrm{me}^{2}\right)$ were quantified in liver over the torpor/arousal cycle (Fig 4.4). Primary antibodies specific that recognized histone H3 only when methylated on specific lysine residues were used (Appendix A). Relative levels of H3K4me increased by $4.9 \pm 0.16$ during EN, compared with EC, before falling again to control levels during ET. Levels of H3K4me then rose during LT (2.0 \pm 0.17 fold higher than EC levels) and increased further in EA to $3.3 \pm 0.13$ fold higher than EC levels $(1.7 \pm 0.07$ fold higher than LT). Levels of H3K4me decreased during LA and remained lower during IA, both near control values. Relative levels of $\mathrm{H} 3 \mathrm{~K} 9 \mathrm{me}^{3}$ decreased from EC levels to $-0.65 \pm 0.03$ fold during EN, and remained significantly lower than EC at all subsequent periods of the torpor/arousal cycle (except for ET), reaching a minimum value during LA (-0.43 \pm 0.04 fold from EC levels). Relative levels of $\mathrm{H} 3 \mathrm{~K} 36 \mathrm{me}^{2}$ rose during the EN period to $2.8 \pm 0.52$ fold higher than EC levels and remained elevated over all other phases of the torpor /arousal cycle, although not significantly different from EC or each other.

\section{Analysis of the methylation status of liver p53}

Relative levels of lysine methylation on p53 were also explored in the liver (Fig 4.5) using antibodies specific to either p53K370me $\mathrm{m}^{2}$ or $\mathrm{p} 53 \mathrm{~K} 372 \mathrm{me}$. Relative levels of p53K370me $\mathrm{m}^{2}$ were unchanged from EC values across EN, ET, LT and EA periods. However, a decrease in relative methylation of the protein occurred during arousal, where LA and IA values were decreased $-0.49 \pm 0.07$ fold and $-0.46 \pm 0.08$ fold compared with EN values, respectively. Relative methylation levels at K372 were also relatively unchanged from EC levels across torpor and arousal except for an increase during IA as compared to p53K372me levels during EN and 
EA. IA levels were $1.9 \pm 0.18$ fold higher than EN levels and $2.1 \pm 0.20$ fold higher than EA levels.

\section{Discussion}

The liver plays a dominant role in the control and maintenance of the body's metabolism during hibernation. While in torpor, the 13LGS survives a prolonged fast and decreasing $\mathrm{T}_{\mathrm{b}}$, in part by decreased mitochondrial metabolism in order to preserve fuels and increase energy preservation as well as to support reductions in transcriptional and translational activity (Staples, 2014; Storey and Storey, 2004b; van Breukelen and Martin, 2002). Subsequently, during arousals from torpor, liver mitochondrial respiration is reinitiated and levels of protein synthesis are restored, presumably in order to promote protein folding, trafficking and turnover, as well as cellular repair mechanisms. Furthermore, a depletion of liver glucose stores during torpor is reversed during arousal, brought about by differential acetylation of phosphoenolpyruvate carboxykinase and liver gluconeogenesis activity (Brown et al., 2013; Hindle et al., 2014; Serkova et al., 2007; Wu and Storey, 2012b). In both hibernators and non-hibernators, the liver is also in control of the mobilization and provision of fuel for extrahepatic tissues in the form of ketone bodies and very-low density lipoprotein particles (Frayn and Arner, 2006; Rui, 2014; Yeh et al., 1995). The action of PTMs on the liver proteome, provides a low-cost and effective method of controlling and stabilizing cells during torpor/arousal cycles, and indeed, this may be observed as differential levels of modifying enzymes and modifying marks within liver protein extractions without the need for regulating protein levels of downstream targets during torpor/arousal cycles (Storey, 1987). The present data attempts to characterize differences in protein levels of KMT enzymes (SMYD2, SET8, SET7/9, RBBP5, ASH2L and G9a) that occur during mammalian hibernation. Differential protein abundance of KMTs in the liver are tied to changes that occur in cellular physiology and signaling cascades that are linked to the facilitation of energy preservation and modulation of the activity or function of proteins which maintain homeostasis in 
non-hibernating animals (Aguilar-Arnal and Sassone-Corsi, 2013; Özdă̆ et al., 2006; Son et al., 2016; Srere et al., 1992). Furthermore, the effects of RPM have a multitude of effects within the liver (Börsch-Haubold et al., 2014; Miao et al., 2007) and so exploring the function of RPM and the associated KMTs during torpor/arousal cycles is key to understanding the cellular processes under the modification's control as well as the responses of hepatic cells to changes in the cellular environment.

The results of this study revealed changes in the relative abundance of specific KMTs at various points over the torpor/arousal cycle, although, overall, differences in the protein levels of the studied KMT enzymes did not seem to follow a common pattern across torpor/arousal cycles (Figures 4.1 and 4.2). Abundance of SMYD2 and SET8 was decreased in in torpor and then significantly increased from these levels during arousal; on the other hand, SET7/9, ASH2L and G9a all showed significant increases during both torpor and arousal, and RBBP5 increased in torpor and decreased during arousal. This is perhaps not surprising given the great deal of variation of targets for each studied KMT enzyme, including those relevant to the control of mammalian hibernation. As an example, both SMYD2 and SET8 showed significant downregulations in LT compared to EC animals, followed by a rebound in protein levels to control amounts in IA. Since differences in KMT enzyme amounts regulate changes in the methylation status of target proteins, it is possible that major shifts in metabolic and protein synthesis activity that occur during torpor and arousal within the liver are also under their control throughout the torpor/arousal cycle.

Looking first at the abundance patterns of SMYD2 and SET8, which decreased during torpor and then increased to control levels in arousal, places these proteins as key to the control of hepatic activity during euthermic conditions, and may emphasize their roles in maintaining downstream modifications during bouts of torpor. Both KMT enzymes may modify histone proteins, although in different ways - SMYD2 monomethylates H3K4 and dimethylates H3K36, while SET8 monomethylates H4K20, all being associated with transcriptional activation via 
enrichment on histones within genes or their promoter regions (Abu-Farha et al., 2008; Beck et al., 2012; Ernst et al., 2011; Wang et al., 2008). By limiting the establishment of activating histone methyllysine modification through decreases in SMYD2 and SET8 during torpor, cells may be restricting the activation of transcription in an epigenetic manner by decreasing the number of sites that transcriptional co-activators may bind to on the chromosome. In this way, decreases in SMYD2 and SET8 abundance would contribute to wide-spread transcriptional suppression characteristic of torpor, and given that decreased transcriptional activity during torpor is advantageous to the hibernator, this mechanism would allow for cellular resource preservation within the liver. On the other hand, control levels of SMYD2 and SET8 during arousal would provide cells a mechanism by which they may increase transcriptional activity in a location specific manner and presumably allow the animal to restore protein amounts of metabolic enzymes required for subsequent torpor bouts. Interestingly, the protein levels of SET8 are significantly increased over control values in LA, and this fluctuation may serve to increase the activity of euthermic metabolic functions or DNA repair mechanisms; non-hibernator studies have shown that p53 binding partner-1 targets nearby chromosomal locations for DNA damage repair mechanisms by recognizing damaged DNA and binding nearby pre-existing H4K20me modifications (Beck et al., 2012; Jørgensen et al., 2013). In this way, increased levels of SET8 in LA may be acting to poise the genome and initiate DNA damage responses in LA and IA, should the need arise. The extent to which either of these mechanisms are employed during hibernation is unexplored and warrants further investigation of these enzymes roles within the liver.

Conversely, the abundance of RBBP5 showed the opposite pattern to SMYD2 and SET8 - RBBP5 protein amount was heightened during entry into torpor and reduced in both EA and IA. As a core member of the mixed-lineage-leukemia (MLL1-4) methyltransferase complex which can target H3K4 for mono-, di- or trimethylation, RBBP5 is largely considered to be a stimulator of cellular activity (Ernst and Vakoc, 2012; Patel et al., 2009). Increased MLL complex KMT activity during EN is further suggested by increased protein levels of complex member ASH2L, 
and differential methylation on H3K4 in the same time point (Figure 4.4, discussed in a later paragraph). Currently, the mechanism by which MLL or MLL-like complexes target areas of the chromosome for transcriptional activation is largely unknown, although appears to require the action of facultative complex members, or recruitment by nuclear hormone receptors including retinoic acid receptors or estrogen receptors (Dou et al., 2005; Lee et al., 2009; Yokoyama et al., 2004). Although increased transcriptional activity while the animal is entering torpor may appear contradictory to the overall state of transcriptional suppression characteristic of torpor, increased RBBP5 and ASH2L activity during this time point may increase the levels of metabolic enzymes required for the upcoming period of torpor. As previously mentioned (in Chapter 1), the abundance of lipolytic enzymes and proteins that aid in cellular lipid uptake were shown to strongly increase during the transition to a torpid state (Epperson et al., 2010a; 2004). A potential epigenetic mechanism which occurs in non-hibernator liver cells is therefore suggested, whereby fluctuations in hepatic lipid reserves would impact the transcriptional status of proteins required for torpor and arousal through lipid hormone nuclear receptor associations with MLL complexes (Garapaty et al., 2009; Garcia-Bassets et al., 2007; Nelson et al., 2009; Yokoyama et al., 2011). Correlated increases in ASH2L and RBBP5 protein levels and $\mathrm{H} 3 \mathrm{~K} 4$ methylation during EN, as well as other findings which show heightened lipolytic enzyme expression in 13LGS liver during the transition to torpor seem to correlate in showing a potential for this epigenetic mechanism to affect cell survival and fuel selection strategies during hibernation (Epperson et al., 2010a). Intriguingly, elevated levels of ASH2L were found not just in EN, as RBBP5 levels were, but throughout the torpor/arousal cycle. KMT activity by ASH2L is typically dependent on heterodimer formation with RBBP5 (Cao et al., 2010; Shinsky et al., 2014); in the absence of complex members, ASH2L acts as a transcriptional co-activators within active areas of the genome through interactions with various transcription factors and $\mathrm{H} 3 \mathrm{~K} 4$ me modifications (Mungamuri et al., 2015; Ullius et al., 2014). The influence of the transcription factor Myc or p53 for example, relies on an interaction with $\mathrm{H} 3 \mathrm{~K} 4$-bound $\mathrm{ASH} 2 \mathrm{~L}$ to regulate the modification status 
of $\mathrm{K} 27$ on $\mathrm{H} 3$ through attraction of $\mathrm{H} 3 \mathrm{~K} 27$-specific demethylases and acetyltransferases in order to further stimulate transcription at downstream chromosomal regions (Tang et al., 2013; Yang et al., 2015). In this way, differential protein levels of ASH2L and transcription factors which bind ASH2L provide a two-layered epigenetic mechanism whereby chromosomes are primed for shifts in transcriptional activity through binding to $\mathrm{H} 3 \mathrm{~K} 4$ which itself shows differential regulation during torpor and arousal (Figure 4.4, discussed in a later paragraph). Whether or not these regulatory mechanisms serve to localize transcriptional activity during 13LGS hibernation remains to be seen.

An upregulation seen in both torpor and arousal is shared between ASH2L and both SET7/9 and G9a. Although both enzymes have a preference for histone targets, the activity of SET7/9 within the nucleus is most likely inhibited during torpor due to its transport into the nucleus being glucose-dependent (previously discussed in Chapter 3; Keating and El-Osta, 2013b). As the activity of gluconeogenesis pathways within the liver are differentially regulated, being suppressed during torpor and stimulated during arousal (Gehnrich and Aprille, 1988; Hindle et al., 2014), SET7/9 may be acting as a histone modifier during LA and IA, when its abundance is also increased. While the scope of this study does not permit an investigation of the exact consequences of increased SET7/9 abundance, the fact that its protein levels are increased during times of increased cellular glucose (Serkova et al., 2007), signify the potential importance of increasing SET7/9's RPM activities during arousal. Conversely, relative levels of H3K4me detected during LA and IA where equal to those seen in control animals, as well as significantly decreased enzymatic activity on the residue in IA compared to control, suggesting that if SET7/9 KMT activity is upregulated in these times, it is not significant enough to manipulate global levels of H3K4me (Figures 4.3 and 4.4). On the other hand, since H3K4me does not increase, increased SET7/9 protein abundance in times of decreased cellular glucose, i.e., during torpor, points to its possible role in regulating non-histone protein methylation (Del Rizzo and Trievel, 2011). For example, the action of cytoplasmic SET7/9 in torpor may regulate transcription factors 
such as NF-kB through methylation of the p65 subunit on lysines 314 and 315

(p65K314meK315me) (Yang et al., 2009). In non-hibernators, methylation of NF- $\kappa$ B by SET7/9 results in transcriptional inhibition via recruitment of protein ubiquitin E3 ligases, a result which typically follows NF- $\kappa$ B's recruitment to and activation of target genes (Saccani et al., 2004). Further alluding to the influence of SET7/9 on NF- $\kappa B$ is the acetylation of lysine 310 of the p65 subunit, which shows negative cross-talk with p65K314meK315me (Yang et al., 2010), and was found to be decreased within 13LGS liver in LT (Rouble and Storey, 2015). A suggested influence by SET7/9 on the methylation status of non-histone proteins during torpor shows that the actions of KMT enzymes may inhibit transcriptional activity through RPM and its interplay with a variety of PTMs. Finally, the regulation of G9a, showed a quite interesting pattern of protein abundance during torpor and arousal. G9a targets H3K9 and weakly targets H3K27 for dimethylation, wherein the former is associated with decreases in transcriptional activity on nearby DNA, while the opposite is true of the latter (Rosenfeld et al., 2009). Protein levels of G9a were strongly enhanced in the transition to torpor, as well as in the final stages of transitioning to euthermia. Since both of these periods are times of major shifts in protein expression and cellular environments (Hindle et al., 2014; Karpovich et al., 2009), the increased levels of chromatin modifying enzymes may not be surprising, when they are likely working to modulate the levels of hibernation-stage-specific protein expression through actions on either H3K9 or H3K27. Furthermore, G9a is a multifaceted regulator of gene expression, having a plethora of substrates and mechanisms by which to exert effects on both downstream gene expression and non-histone protein modification-status (Shankar et al., 2013).

In order to characterize the effects that differential KMT protein amounts may have on histone proteins, the methyltransferase activity specific to $\mathrm{H} 3 \mathrm{~K} 4, \mathrm{H} 3 \mathrm{~K} 9$ and $\mathrm{H} 3 \mathrm{~K} 27$ lysine residues, as well as the methylation status of $\mathrm{H} 3 \mathrm{~K} 4 \mathrm{me}, \mathrm{H} 3 \mathrm{~K} 9 \mathrm{me}^{3}$ and $\mathrm{H} 3 \mathrm{~K} 36 \mathrm{me}^{2}$ throughout the torpor/arousal cycle was investigated (Figures 4.3 and 4.4). Methyltransferase activity on H3K4 was unchanged throughout torpor, and was inhibited in IA according to KMT activity 
measurements, although $\mathrm{H} 3 \mathrm{~K} 4$ methylation on histone tails extracted from 13LGS tissue was actually seen to be increased in EN and LT, as well as in EA, compared to H3K4me levels observed in control animals. The disparity seen between H3K4 methyltransferase activity and actual levels of H3K4 likely suggests the interplay of other histone modifications, that are absent in the in-vitro methyltransferase activity assays and which either stimulate or inhibit H3K4 methylation within the cell. The actual mechanism by which global H3K4 activity is being stimulated, is unfortunately outside the scope of this study, however, functional interactions between H3K4 methylation and Arg-2 dimethylation, Tyr-3 phosphorylation and Lys-9 di- or trimethylation all also on histone H3, have been shown to occur (Binda, 2013) and may play a role in poising or rejecting $\mathrm{H} 3 \mathrm{~K} 4$ methyltransferase activity during torpor and arousal. The fact that $\mathrm{H} 3 \mathrm{~K} 4 \mathrm{me}$ levels are upregulated during torpor does seem contradictory to the hibernator's need to preserve cellular resources in these times, although increases in $\mathrm{H} 3 \mathrm{~K} 4 \mathrm{me}$ may be signaling the cell's need for transcription of torpor-specific proteins under the control of H3K4me-mediated transcriptional activation. Studies investigating the role of H3K4 methylation within the liver in the context of human diabetes and obesity have shown that levels of H3K4me are enhanced in diabetic conditions, and that the effect may be attenuated through treatment with glucagon-agonists (Muka et al., 2016; Tu et al., 2015). Given that hibernating mammals are reliant on lipid catabolism during torpor, as well as aberrant endocrine signaling which mirrors that seen in diabetes (Bauman et al., 1987; Wu et al., 2013), a similar mechanism may be utilized by the liver to accommodate shifts in metabolic fuel selection through increased levels of H3K4me. Similarly, levels of the transcriptional-activating H3K36me $\mathrm{m}^{2}$ were also heightened during EN, although in this case, activation of gene activity may be centered around the liver's need to support gluconeogenesis throughout the winter to combat decreased levels of circulating glucose (Gehnrich and Aprille, 1988; Pan et al., 2012; Williams et al., 2011). Changes to the status and overall levels of histone modification may be altered as a result of changes in metabolic activity during torpor and arousal; as observed in diabetic cells from non-hibernators, 
conditions of hyperglycemia, for instance, lead to an increase of activating histone modifications on the gene promors of the NF- $\mathrm{kB}$ p65 subunit and glucose transportor-2 within the liver (Jayaraman, 2012; Mosley and Ozcan, 2003; Reddy et al., 2015). The regulation of enzymes involved in beta-oxidation during torpor and arousal are likely also modulated by the effects of histone methylation, given that over 300 genes have been shown to be effected by differential histone compaction and chromatin relaxation (Jun et al., 2012; Leung et al., 2014). On the other hand, increased methyltransferase activity on the transcriptional-repressive $\mathrm{H} 3 \mathrm{~K} 9$ residue is seen during torpor, although not significantly compared to EC animals. Widespread suppression of the genome is expected during torpor, as increased methyltransferase activity would facilitate the cell's suspension of transcriptional activity. During IA however, methyltransferase activity on $\mathrm{H} 3 \mathrm{~K} 9$ was reduced compared to activity measurements in animals transitioning to torpor, suggesting that H3K9-mediated repression is maintained throughout torpor, and until a concomitant reduction in methyltransferase activity is seen in the later stages of arousal. Levels of $\mathrm{H} 3 \mathrm{~K} 9 \mathrm{me}^{3}$ were significantly decreased on histone tails in each stage of torpor and arousal compared to control animals however, this suggests that negative crosstalk between H3K9specific methyltransferases and other histone PTMs is occurring, similar to the case described for H3K4-mediated gene activation (Binda et al., 2010). Although the downstream effects of H3K9mediated gene repression were not investigated in this study, it could be that decreased $\mathrm{H} 3 \mathrm{~K} 9 \mathrm{me}^{3}$ plays a role in promoting cellular senescence pathways through decreases in repressive histone modifications, and a concurrent increase in activating histone modifications in the genic regions of cellular senescence promoting proteins (Zhang et al., 2012b). Opposite to the pattern seen in H3K9 methyltransferase activity, an elevation of methyltransferase activity was seen on H3K27 in IA compared to control animals. Increased methylation activity directed at H3K27 may be advantageous to the animal at this time by regulating the transcription of essential gene products. Unfortunately, detection of $\mathrm{H} 3 \mathrm{~K} 27 \mathrm{me}$ was impossible in liver due to negligible amounts of immunoblot band detection, making speculation about the results of decreased H3K27 
methyltransferase activity difficult since it is unknown if $\mathrm{H} 3 \mathrm{~K} 27$ is methylated during torpor and arousal on histone tails, as well as to what degree the residue is methylated. However, overall patterns of regulation imposed on histone methylation during torpor provides further evidence of functional correlations between epigenetic mechanisms and the hibernation phenotype, at least partly controlled by the actions of histone methylation.

Finally, the methylation status of non-histone proteins within the liver was investigated to understand whether differential methylation may be found on cytoplasmic proteins and hence facilitate the regulation of torpor arousal cycles in a non-epigenetic manner. Within the liver, the transcription factor p53 is a crucial regulator of apoptosis, DNA damage repair and cell cycle checkpoint control. The relative methylation status of the p53 transcription factor, at K370 (p53K370me ${ }^{2}$ ) or at K372 (p53K372me), both of which enhance p53's stability on chromosomes, were found to be differentially modified during torpor and arousal, although in opposite ways (Figure 4.5). Decreased levels of p53K $370 \mathrm{me}^{2}$ late in the arousal period and in IA were observed, while levels of p53K372me on the other hand, were increased in IA, all compared to EC animals. The significance of inhibitory cross-talk between the two modifications is a likely interaction, given their proximity, and would fit with findings showing an interaction between methylation of the two lysine residues in a number of non-hibernator studies (Carr et al., 2012; West and Gozani, 2011). Briefly, monomethylation activity by SET7/9 at p53K372 in response to DNA damage, stabilizes an interaction of p53 with chromatin, and correlates with increased acetylation of p53 at nearby lysine residues to cause increases in transcriptional activity (Chuikov et al., 2004; Ivanov et al., 2007). Furthermore, electrostatic interference between p53K372me and SMYD2, which monomethylates p53K370 and represses p53 target gene activation, has been shown to occur and because monomethylation at $\mathrm{K} 370$ is required for its subsequent dimethylation, the latter modification is also inhibited (Huang et al., 2006; 2007). These findings together with the results from this study underscore the role of methylation in increasing p53 transcriptional activity while the 13LGS is in IA. Within the context of 13LGS liver, increased 
regulation of p53 activity during IA seem to be a common finding (Hefler et al., 2015; Pan et al., 2014; van Breukelen, 2016); fluctuations in mRNA, and protein abundance of p53 and its coregulators and binding partners have been shown to occur, wherein IA protein levels of p53 were maximized. At the same time, DNA-binding of p53 in IA was only moderately heightened, as was RNA-polymerase II recruitment to p53-upregulated target genes. Surprisingly however, actual mRNA transcript levels of p53-upregulated target genes were actually decreased in IA, which the authors suggest is typical of p53 target transcripts being specifically targeted for concomitant degradation as well as ineffective regulation of transcription during torpor arousal cycles (Pan et al., 2014). Observations of differential methylation of p53 during torpor and arousal would seem to agree with the mentioned study, in that the stabilization of p53 on the chromosome during IA is promoted and poises the transcription factor for activity.

In terms of the current research, it would appear that RPM by KMT enzymes is characteristic of the liver's response to shifts in activity and environment that are typical of torpor and arousal cycles. The presented results identify significant fluctuations of six well characterized KMT enzymes. Furthermore, changes in the methyltransferase activity and the methylation status imposed upon histone $\mathrm{H} 3$ or the p53 transcription factor at various points throughout torpor and arousal were investigated. Similarly, correlated abundance patterns of KMT enzymes that serve similar functions may underscore the importance of certain methylation-modifications to the hibernator phenotype, such as correlated increases in SMYD2 and SET7/9 during IA which point to the significance of their combined functions of regulating genomic activity through the modification of histone proteins. In conclusion, by providing a preliminary characterization of the enzymes in the current system, these data correlate changes in KMT abundance and activity to control over cellular activity during hibernation, in either an epigenetic manner through targeting histone proteins, or in a way that targets other proteins which themselves may serve to regulate transcriptional activity. Finally, by presenting examples of the variety of activities and mechanisms that RPM may regulate within the liver, suggestions of the diverse functional 
consequences that may be achieved through the regulation of this PTM during hibernation are provided. 

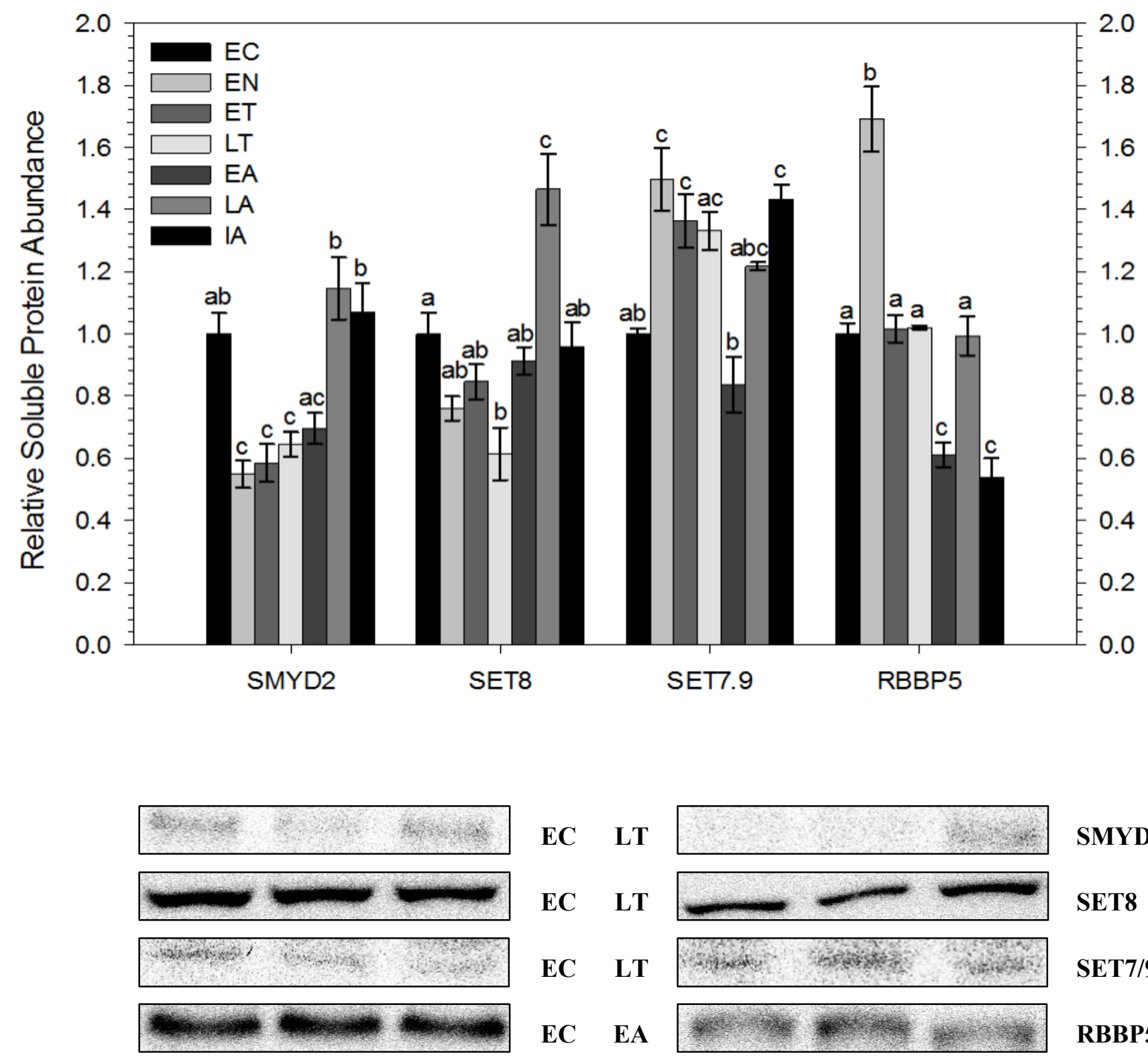

SMYD2

SET8

SET7/9

RBBP5

Figure 4.1: Relative protein abundance of SMYD2, SET8 SET7/9 and RBBP5 in liver of $I$. tridecemlineatus over the torpor/arousal cycle along with three representative immunoblot protein bands for EC and LT sampling points (labelled between the blots). Sampling points are: euthermic in the cold room (EC), entrance into torpor (EN), early torpor (ET), late torpor (LT), early arousal (EA), late arousal (LA) and interbout arousal (IA); see Chapter 2: Materials and Methods for more extensive definitions. Histograms show mean standardized band densities ( \pm SEM, $n=4$ independent trials on tissue from different animals). Protein bands were standardized against the summed intensity of a group of Coomassie-stained protein bands from the same sample lane. Data were analyzed using a one-way ANOVA with a post hoc Tukey test. Different letters denote values that are significantly different from each other $(\mathrm{p}<0.05)$. 


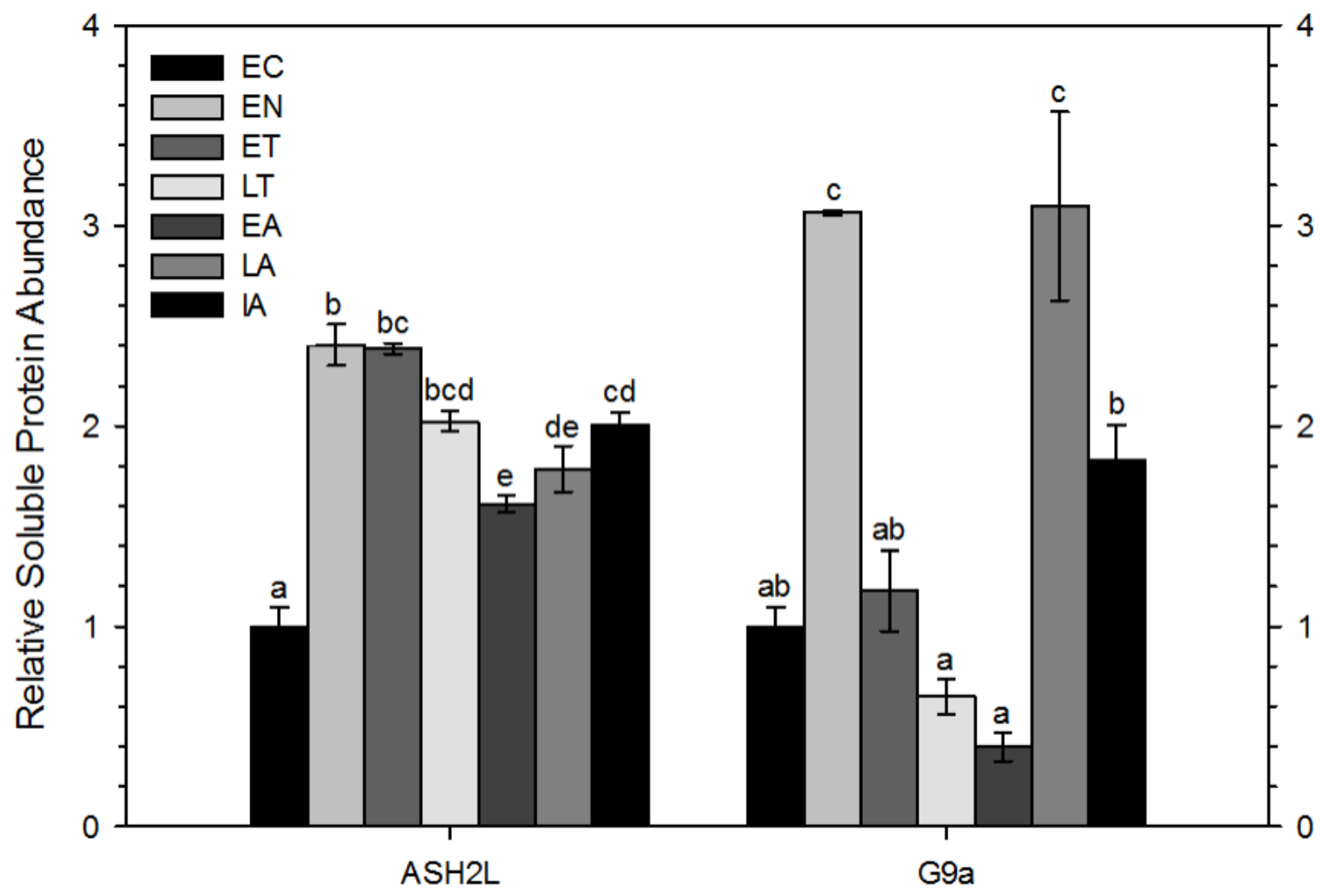

4

3

2 


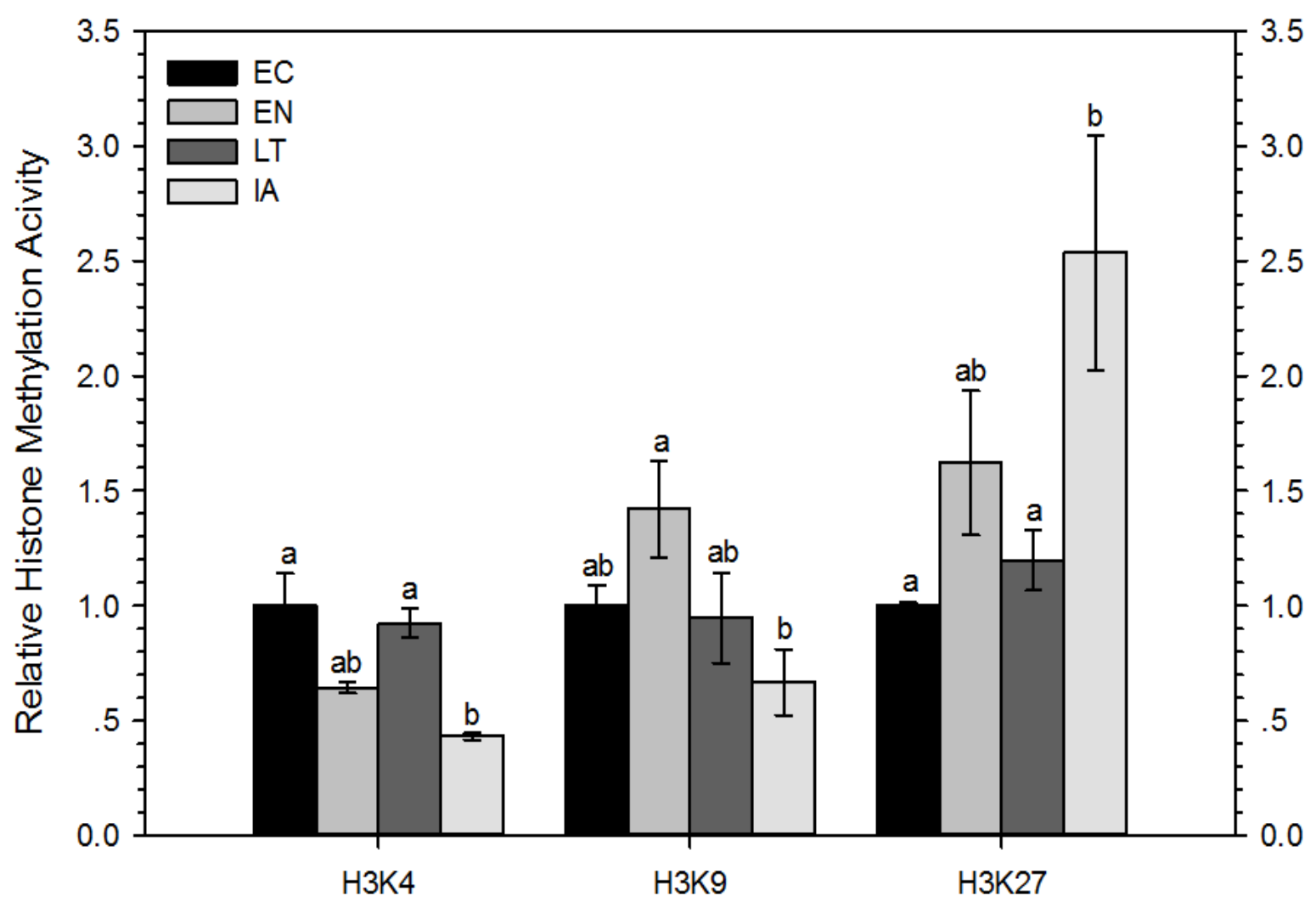

Figure 4.3: Relative histone methyltransferase activity at H3K4, H3K9 and H3K27 in skeletal muscle total soluble protein extracts from I. tridecmlineatus comparing euthermic in the cold room (EC) to entry to torpor (EN), late torpor (LT) and interbout arousal (IA) time points of the torpor/arousal cycle. Histograms show mean amount of methyllysine produced per hour per weight protein added $(\mathrm{ng} / \mathrm{h} / \mathrm{mg}) \pm \mathrm{SEM}, \mathrm{n}=3-4$ independent assays on tissue from different animals. Histone methyltransferase activity at $\mathrm{H} 3 \mathrm{~K} 4, \mathrm{H} 3 \mathrm{~K} 9$ and $\mathrm{H} 3 \mathrm{~K} 27$ was found to be 2.00 $\pm 0.15 \mathrm{ng} / \mathrm{h} / \mathrm{mg}, 1.24 \pm 0.09 \mathrm{ng} / \mathrm{h} / \mathrm{mg}$ and $5.04 \pm 0.02 \mathrm{ng} / \mathrm{h} / \mathrm{mg}$, respectively in control animals, with all other time points shown relative to control activity. Data were analyzed using a one-way ANOVA with a post hoc Tukey test. Different letters denote values that are significantly different from each other $(p<0.05)$. 

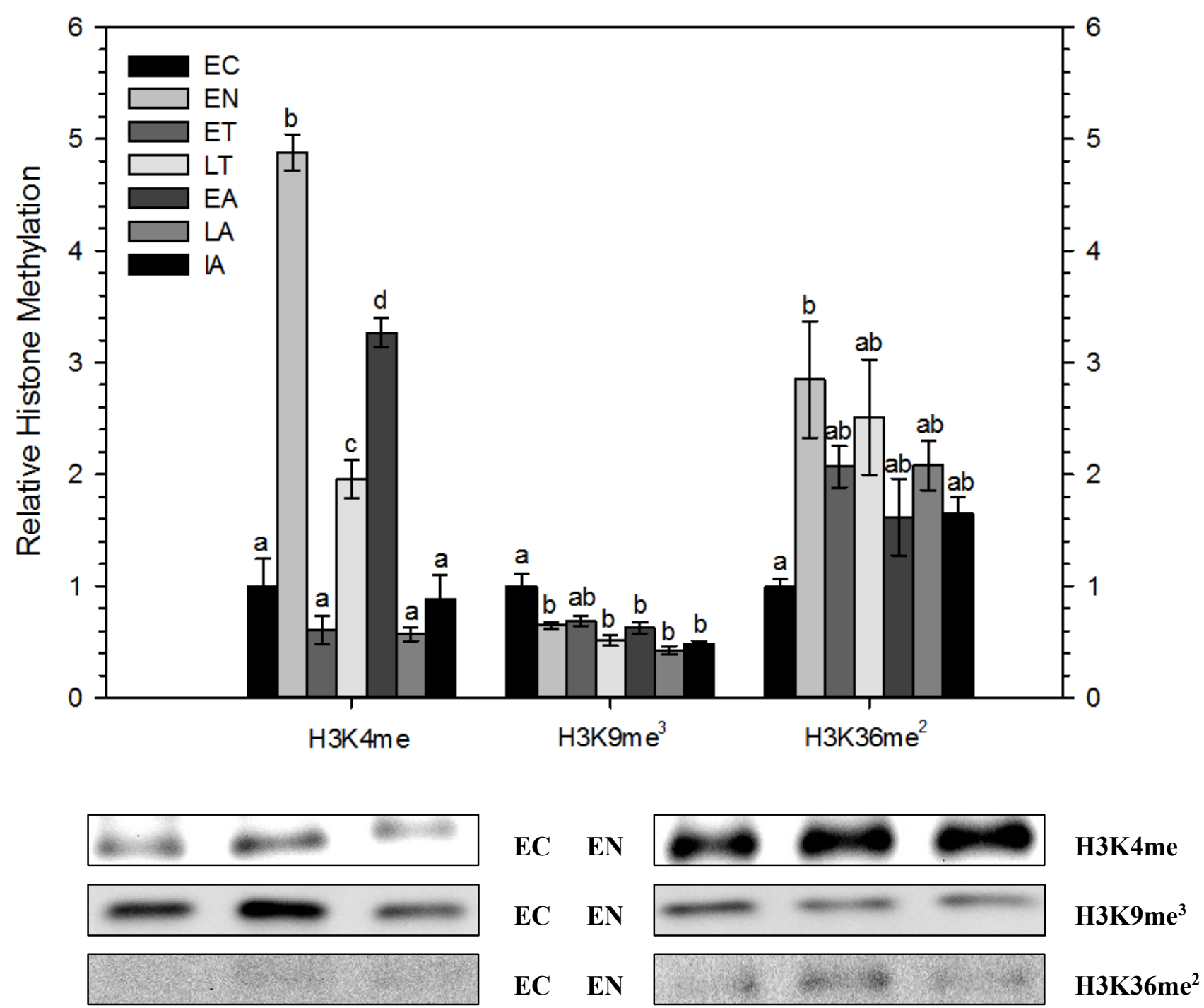

Figure 4.4: Relative amounts of histone $\mathrm{H} 3$ that is monomethylated at $\mathrm{K} 4$ ( $\mathrm{H} 3 \mathrm{~K} 4 \mathrm{me}$ ), timethylated at $\mathrm{K} 9\left(\mathrm{H} 3 \mathrm{~K} 9 \mathrm{me}^{3}\right)$ or dimethylated at $\mathrm{K} 36\left(\mathrm{H} 3 \mathrm{~K} 36 \mathrm{me}^{2}\right)$ in liver of I. tridecemlineatus over the torpor/arousal cycle along with three representative immunoblot protein bands for EC and EN sampling points (labelled between the blots). All other information is as in Fig. 4.2. 

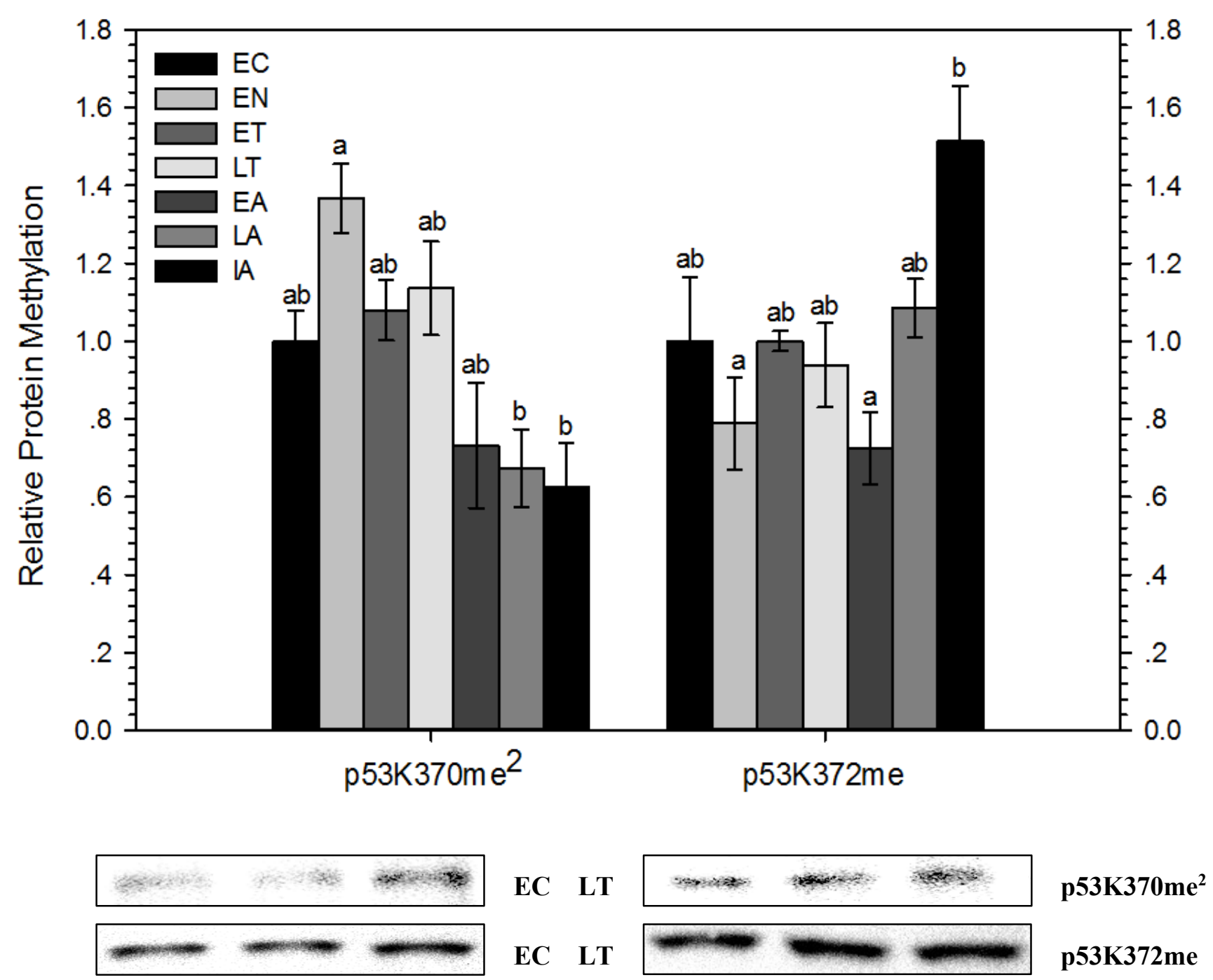

Figure 4.5: Relative amounts of p53 that is dimethylated at $\mathrm{K} 370\left(\mathrm{p} 53 \mathrm{~K} 370 \mathrm{me}^{2}\right)$ or monomethylated at $\mathrm{K} 370$ (p53K370me) in liver of I. tridecemlineatus over the torpor/arousal cycle along with three representative immunoblot protein bands for EC and LT sampling points (labelled between the blots). All other information is as in Fig. 4.2. 


\section{Chapter 5}

General Discussion 
Mammalian hibernation is an interesting phenomenon that provides animals with a chance to survive through periods of fasting and major metabolic rate depression, as well as shifts in physiology which in most cases would be lethal to non-hibernators yet is tolerated numerous times each winter in hibernating organisms. In order to cope with such changes and survive the winter, mammals, such as the 13LGS, have evolved a number of controls over gene transcription, protein synthesis, metabolic strategies and physiological functions, which ensure the animal's survival and overwintering success. For instance, in the face of winter-time environments (i.e., decreased food abundance and increased costs for homeothermy), the 13LGS's tissues increase their reliance on protective mechanisms, including enhanced antioxidant defences and cellular preservation strategies, chromatin structure modifications and increased expression of hibernation-responsive genes. These changes are necessary in order to maintain essential cellular functions during hibernation as well as allow for the full recovery of functions following the winter season. Furthermore, the 13LGS is able to preserve bodily fuel stores by manipulating the use and substrates of catabolism, reducing energy needs. Similarly, a temporary shutdown of a variety of metabolic pathways during a season-long fasting period is experienced, decreasing cytotoxic stresses and limiting the amount of stored fuel that the animal needs to store internally (Carey et al., 2003; Storey, 2010). Indeed, research investigating the cellular and physiological adaptations that permit the 13LGS and other mammals to undergo torpor/arousal cycles is beneficial to our understanding of the mammalian cell's response to stressful environments.

In order to intricately regulate the massive shifts in phenotype that occur between torpid and aroused animals, biochemical mechanisms that target a wide variety of cellular pathways and processes are needed by the animal to control most aspects of cellular functions, and regulate their activity in response to changes in cellular environments. Given the diverse array of processes that protein post-translational modifications are in control of during cellular stress (e.g., chromatin dynamics, cellular structural integrity and stability and transcription factor-readiness) these regulators of cellular activity must be responsible for a large role in the shift in enzyme 
activity and protein stability that plays an essential role in regulating hibernation (Duan and Walther, 2015; Fan et al., 2015; Morin and Storey, 2009; Pałyga, 2012). As introduced in

Chapter 1, the roles of protein kinases and protein phosphatases are involved in controlling the activities of a number of glycolytic enzymes and transcription-factor binding abilities during mammalian hibernation (Abnous et al., 2008; Bell et al., 2014; Logan et al., 2016a; Storey, 1997). Similarly, the actions of protein deacetylases causing differential levels of acetylation have been found to be involved in regulating torpor/arousal cycles in the 13LGS (Rouble and Storey, 2015). Protein PTMs are undoubtedly necessary for regulating and maintaining homeostasis, and this is indeed the case in times of altered cellular environments, as seen during torpor/arousal cycles.

Given the rapid and robust regulatory capacity displayed by protein PTMs, as well as the ubiquity seen in the roles for RPP and RPA in torpor/arousal cycles, it would appear that other similar modifications would be just as essential in the regulation of mammalian hibernation - as the current study has attempted in the context of RPM. As discussed previously, RPM has been shown to mediate control over an incredibly diverse range of protein functions in non-hibernating species, with many roles that may accompany, complement or even interfere with RPP and RPA (Alam et al., 2015; Bannister and Kouzarides, 2011; Berger, 2002; Biggar and Li, 2015; Kouzarides, 2007). For example, RPM is commonly involved in regulating chromatin dynamics via histone modifications, as well as in regulating transcription factor binding affinities and the mobilization of cellular protective responses (Alam et al., 2015; Biggar and Li, 2015; Binda, 2013). All of these processes are also affected by RPP and RPA and are relevant to hibernators' success in controlling torpor/arousal cycles. Evidence does not currently exist which demonstrates a role for RPM in the context of mammalian hibernation, and such data would be essential to highlighting the regulatory capacities of protein PTMs for the 13LGS and during torpor/arousal cycles. By understanding how ground squirrel skeletal muscle and liver, two tissues of extreme importance to the hibernator during torpor and arousal, adjust their cellular 
mechanisms to maintain function while responding to changing workloads (i.e., in euthermia vs during torpor), we can gain increased understanding of the adaptive mechanisms at play in mammalian tissues.

The work presented in this thesis hypothesized that, given the known roles of RPP and RPA in regulating torpor/arousal cycles in mammals, and given the regulatory nature of protein PTMs, RPM is correlated with the cellular changes that occur during mammalian hibernation. By providing a preliminary characterization of some of the important KMTs involved in RPM, including their activity levels as well as the methylation status of their downstream targets in skeletal muscle and liver in 13LGS during mammalian hibernation, important insight may be gained as to the relevance and consequences of RPM during torpor/arousal cycles. In spite of reduced metabolic activity, skeletal muscle tissue must maintain function and preserve cellular integrity during transitions into and out of extended periods in deep torpor while avoiding cytotoxic stresses. On the other hand, the liver of the hibernating 13LGS relies more on reductions in metabolic functions that are not needed during the torpid state, in adaptations which serve to conserve metabolic energy. As evidenced in Chapter 3, differential RPM correlates with enhanced regulation over the activation of hibernation-specific responses in skeletal muscle, while Chapter 4 illustrates that RPM may be correlated with times when reductions in transcriptional activity within the liver is prominent. These results, when taken together, show that differential methylation is seen in each of the time points of the torpor/arousal cycle and between two tissues that serve opposite functions in enhancing the animals' survival.

The results also seem to suggest that the abundance and ultimately the actions of KMT enzymes are differentially regulated between skeletal muscle and liver tissues, suggesting tissuespecific mechanisms of regulation are in control of KMT enzyme levels. Interestingly, both SMYD2 and SET8 showed quite different protein abundance patterns throughout torpor and arousal. Protein levels of SMYD2 showed increases during torpor and decreases during arousal in skeletal muscle, while the opposite was true in liver, decreasing during torpor and increasing 
during arousal. Given that methyltransferase activity by SMYD2 targets both H3K4 and H3K36, causing downstream transcriptional activation, opposite patterns of protein abundance may not be surprising given the differences in physiological function of skeletal muscle and liver tissues during torpor and arousal. As described in Chapters 1 and 3, the heightened amounts of myoprotective factors during torpor are key to the stability and survival of skeletal muscle cells during bouts of torpor, when avoidance of muscle atrophy in the face of prolonged muscle unloading is required (Andres-Mateos et al., 2012; Hindle et al., 2015; Tessier and Storey, 2010). On the other hand, as described in Chapters 1 and 4, during arousal within the liver has an important role in providing fuel for the body during euthermic periods. The results contained in this thesis highlight roles for correlations of KMT enzyme abundance with changes in cellular regulation that are typical of both torpor and arousal in skeletal muscle and liver tissues. For instance, it may be that liver SMYD2 is acting to increase the transcription of important regulators of metabolism and cytoprotection within the liver, given the shift in gluconeogenic activity, as well as increases in oxidative phosphorylation, during arousal (Epperson et al., 2010a; Ni and Storey, 2010). Similarly, abundance patterns of SET8 suggest that increases in protein amount during arousals are also key to the shift that takes place within the liver during LA. Unchanged protein abundance from control animals in skeletal muscle provide further evidence of the tissue-specificity of the enzymes action within hepatic cells. Given that the activity of SET8 targets H4K20 for methylation (essential for DNA damage repair responses and genome stability), its increased abundance within liver during arousal may correlate with downstream increases in transcriptional activity (Beck et al., 2012; Botuyan et al., 2006; Jørgensen et al., 2013), the deposition of H4K20me modifications on chromosomes, perhaps reflecting a preparatory mechanism by which other transcriptional activating modifications are primed and enhanced (Schwartz et al., 2009; Wagner and Carpenter, 2012). Given that the unnecessary expenditure of energy during mammalian hibernation would be life-threatening to the animal, a variety of mechanisms must be intricately employed to enhance the regulation over energy- 
intensive processes such as protein synthesis. In this respect, the differential abundance of the RBBP5 and ASH2L heterodimer components is somewhat surprising. One would expect that as members of a functional complex, strong increases in one component would be linked to the other in times of heightened energy preservation, although enhanced abundance of ASH2L within the liver was found in the absence of enhanced RBBP5 abundance, and opposite to the protein abundance data observed in skeletal muscle. Taken together, these findings may be evidence of the influence of independent $\mathrm{ASH} 2 \mathrm{~L}$ as a non-catalytic binding partner of regulatory transcription factors and complementary chromatin modifiers. Without partner complex members, ASH2L may bind pre-existing $\mathrm{H} 3 \mathrm{~K} 4$ methyl-modifications to recruit and promote the activities of histone acetyltransferases and demethylase enzymes that are specific to inhibitory methylation modifications (Tang et al., 2013; Yang et al., 2015). Although speculation, epigenetic regulation of transcription by KMTs through non-catalytic activities has been shown in non-hibernators to regulate the synthesis of proteins from numerous pathways during cellular growth or stress (Estève et al., 2006; Johnstone and Baylin, 2010; Manzur and Zhou, 2005; Sarvan et al., 2011).

Finally, the actions of the epigenetic repressor G9a, showed similar patterns in protein amount between the two studied tissues, but interestingly, seemed to be upregulated within torpor and arousal during opposite time points; enhanced G9a levels during torpor within skeletal muscle were limited to ET, while liver G9a levels were enhanced in EN. This pattern of regulation on protein levels of G9a between tissues suggests that the actions of this KMT may in fact be similar between tissues, as G9a strongly targets H3K9 for methylation, repressing gene transcription in the transitory stages of torpor (EN and ET), although temporally regulated through differences in protein interactions perhaps. Taking these tissue-specific differences in protein abundance together with global $\mathrm{H} 3 \mathrm{~K} 9$ methyltransferase activity measurements during the corresponding time points and tissues allows an estimation of the effect that G9a may play on the suppression of transcription. Liver $\mathrm{H} 3 \mathrm{~K} 9$ methyltransferase activity is significantly increased in EN, correlating with G9a protein amount, while skeletal muscle $\mathrm{H} 3 \mathrm{~K} 9$ methyltransferase activity 
is significantly reduced during LT, correlating with a significant fall in protein amount from ET. Given the already established roles of G9a in the modification of both histone and non-histone proteins within both skeletal muscle and liver (Claycombe et al., 2016; Rao et al., 2016; Shankar et al., 2013), it is reasonable to suggest that observed G9a fluctuations might be related to some of those roles, although impossible to pin the activity of G9a to one specific function given the scope of this study. On the other hand, detection of histone tail methylation modifications in protein samples extracted from 13LGS tissue, provide a better estimation of the actions of KMT enzymes during torpor and arousal. Since histone PTM crosstalk is such a prominent mechanism of regulating transcriptional activity, differential methyltransferase activity alone does not provide clues as to whether or not the activity of KMT enzymes is altered by pre-existing histone modifications. Differential levels of the repressive modification $\mathrm{H} 3 \mathrm{~K} 9 \mathrm{me}^{3}$ that were seen in a tissue-specific manner, largely decreased within liver across torpor and arousal, while showing a torpor-specific increase and arousal-specific decrease within skeletal muscle. Within skeletal muscle, fluctuations in $\mathrm{H} 3 \mathrm{~K} 9 \mathrm{me}^{3}$ would correlate with a suppression of genomic activity during torpor, while its decrease would correlate with a reversible enhancement of transcription in euthermic periods. Conversely, within the liver, strong increases in $\mathrm{H} 3 \mathrm{~K} 4 \mathrm{me}$ may be working to suppress the effects of transcriptional-suppression on torpor-specific genes. Interactions between methylated $\mathrm{H} 3 \mathrm{~K} 4$ and repressive methyl modifications on $\mathrm{H} 3 \mathrm{~K} 9$, which is decreased during both torpor and arousal within liver, may be possible given observations of histone cross-talk between the two modifications (Chaturvedi et al., 2012; Nightingale et al., 2007). It would appear that by enhancing levels of activating histone modifications during torpor and arousal the genome may be poised for rapid shifts in protein synthesis activity, required to provide metabolic resources to the body's organs during arousal (Hindle et al., 2014; Miao et al., 2007; Serkova et al., 2007; Tu et al., 2015). The methylation status of $\mathrm{H} 3 \mathrm{~K} 36 \mathrm{me}^{2}$ was also investigated, and shown to be heightened in both skeletal muscle and liver, in LA in the former tissue, but throughout torpor and arousal in the latter tissue. As mentioned, the deposition of activating histone modifications 
within the liver seems to be a common finding, as levels of $\mathrm{H} 3 \mathrm{~K} 4 \mathrm{me}$ are also increased during torpor and in EA. Increases in activating histone modifications within the liver may correlate with regulation of beta-oxidation enzyme expression, as well as the increased synthesis of proteins involved in promoting a transition to cellular-senescence which is seen during torpor (Jun et al., 2012; Wu and Storey, 2012b; Zhang et al., 2012b). Conversely, restriction of H3K36me ${ }^{2}$ to LA in skeletal muscle however, shows that differential levels of histone PTMs are actively regulated between hibernation time points and correlate with increasing genomic activity as the squirrel rewarms skeletal muscle tissue to euthermic vales. Since the 13LGS is actively increasing levels of protein synthesis during arousal periods, and even moreso towards the later stages of arousal, increased levels of skeletal muscle $\mathrm{H} 3 \mathrm{~K} 36 \mathrm{me}^{2}$ in LA likely contribute to the organization of genic regions, as well as the repression of aberrant transcription by RNA polymerase II (Bartke et al., 2010; Schwartz et al., 2009). In the future, levels of other methylated histone markers (e.g., H3K14, H3K79, H4K20 or H2BK5), as well as other histone modifications, should be investigated to further elucidate their role in regulating torpor and arousal, as well as their genomic localization which may help to pinpoint the downstream effects of relevant histone modifications.

In order to investigate RPM and tissue-specificity of KMT activity on non-histone proteins, methylation of skeletal muscle HSP90 and liver p53 were characterized. Differential levels of RPM on methylated proteins that were measured provide further evidence to support the notion that methylation of lysine residues contributes to the overall regulation of hibernation as well as insight into the specific mechanisms by which KMTs effect cellular regulation. For instance, methylation on HSP90 was shown to be differentially regulated during arousal, suggesting a requirement for HSP90-SMYD2 complexes formation, stimulated by HSP90 KMT activity by SMYD2, that would stabilize sarcomere proteins during times of heightened shivering thermogenesis (Voelkel et al., 2013). In another experiment, an investigation of p53 lysine methylation within the liver on two separate residues, which each exert congruent effects on 
target gene activation, found that inhibitory interplay between the two modifications led to an increase of p53K372me, under the control of SET7/9, and a concomitant decrease in $\mathrm{p} 53 \mathrm{~K} 370 \mathrm{me}^{2}$. Given that the function of $\mathrm{p} 53$ has important roles in the transcriptional regulation of downstream target genes, a mechanism by which KMT enzymes may increase transcription through RPM of transcription factors during hibernation is suggested. Furthermore, the differential methylation states of HSP90 and p53 establish the roles of RPM by KMT enzymes as characteristic of torpor and arousal cycles and suggest that a "hibernator lysine-methylome" may be involved in changes to both cellular and physiological phenotypes that are required during mammalian hibernation. As the actions of KMT enzymes on a number of proteins are known to regulate a plethora of cellular mechanisms in almost all cells and almost all animals (Biggar and Li, 2015; Calpena et al., 2015; Kouzarides, 2007), it is likely that the role of RPM in regulating hypometabolic states is not exclusive to mammalian hibernation. Full-scale characterization of the targets of lysine methylation as well as their methylation states should be attempted in the future, possibly through two-dimensional difference gel-electrophoresis and mass-spectrometry, so that life science research may understand the roles and significance of lysine methylation to cellular regulation of altered environments, such as seen in mammalian hibernation. Additional understanding of the roles of RPM by KMT enzymes may also be found through the enzymes purification from hibernator tissues, and assays of KMT activity, as well as post-translational modification quantification.

\section{Conclusion}

Ictidomys tridecemlineatus may survive the winter through drastic reductions of a variety of biological activities in a feat known as hibernation. This thesis provides the preliminary characterization of several lysine methyltransferase enzymes, a family of chromatin modifiers whose function and activity not yet been characterized within the context of mammalian hibernation. The research that was presented shows that the influences of lysine methylation are 
involved in the regulation of torpor/arousal cycles, in mechanisms that may be encompassed by methylation of histone proteins or non-histone proteins, both of which may exert effects on the genome and regulate transcriptional activity in this way. The methylation status of several KMT enzyme targets was also characterized, and results showed that differential RPM on histone proteins are evident throughout torpor/arousal cycles - typically correlated to methyltransferase activity measurements specific to histone-lysine residues. Differential levels of RPM were also found on non-histone targets that may be stimulated to regulate a diverse array of cellular processes following lysine methylation, including the modulation of cellular survival mechanisms. The current data serves to provide a starting point for research that further investigates the roles of RPM by KMT enzymes in mammalian hibernation, as well as other studies of cellular responses to altered environments. Suggested directions for future studies include the characterization of protein levels as of yet unstudied KMT enzymes during torpor and arousal, as well as the isolation and characterization of individual KMT enzymes. Studies such as these would be able to determine those KMT enzymes which likely play crucial roles in the regulation of the hibernation phenotype. Further investigation of the methylation status of downstream targets of KMT enzyme activity would also be an interesting avenue for further research characterizing the molecular mechanisms by which cells respond to torpor and arousal. Large-scale mass-spectrometry studies would be more suited to the latter experiments due to the substantial number of KMT enzyme targets that may be modified, as well as the ability to simultaneously detect different degrees of methylation on lysine residues. Further studies should also attempt to characterize of the chromosomal localization of KMT enzymes as well as their methylated histone targets through chromatin-immunoprecipitation, in order to understand the downstream effects of histone methylation on gene transcription and chromatin compaction. In these ways, future studies would illuminate the cellular implications of RPM of both histone and non-histone targets as well as determine the actual effects of RPM on transcriptional regulators. Given the ubiquity between RPMs role in mammalian hibernation and that played by other 
PTMs, including RPP and RPA, the functional significance of lysine methylation to torpor/arousal cycles is likely to be wide-spread. Despite the lack of these future studies, this thesis is successful in attempting to characterize the roles of KMT enzymes in mammalian hibernation as well as establishing novel roles for this PTM, showing that lysine methylation is characteristic to mammalian hibernation. 


\section{References}


Abnous, K., Dieni, C.A., and Storey, K.B. (2008). Regulation of Akt during hibernation in Richardson's ground squirrels. BBA-Gen Subjects 1780, 185-193.

Abu-Farha, M., Lambert, J.-P., Al-Madhoun, A.S., Elisma, F., Skerjanc, I.S., and Figeys, D. (2008). The tale of two domains: proteomics and genomics analysis of SMYD2, a new histone methyltransferase. Mol. Cell Proteomics 7, 560-572.

Aguilar-Arnal, L., and Sassone-Corsi, P. (2013). The circadian epigenome: how metabolism talks to chromatin remodeling. Curr Opin Cell Biol 25, 170-176.

Alam, H., Gu, B., and Lee, M.G. (2015). Histone methylation modifiers in cellular signaling pathways. Cell. Mol. Life Sci. 72, 4577-4592.

Allan, M.E., and Storey, K.B. (2012). Expression of NF-кB and downstream antioxidant genes in skeletal muscle of hibernating ground squirrels, Spermophilus tridecemlineatus. Cell Biochem Funct 30, 166-174.

Allis, C.D., Berger, S.L., Cote, J., Dent, S., Jenuwien, T., Kouzarides, T., Pillus, L., Reinberg, D., Shi, Y., Shiekhattar, R., et al. (2007). New nomenclature for chromatin-modifying enzymes. Cell 131, 633-636.

Andres-Mateos, E., Mejias, R., Soleimani, A., Lin, B.M., Burks, T.N., Marx, R., Lin, B., Zellars, R.C., Zhang, Y., Huso, D.L., et al. (2012). Impaired skeletal muscle regeneration in the absence of fibrosis during hibernation in 13-lined ground squirrels. PLoS ONE 7, e48884.

Andrews, M.T., Squire, T.L., Bowen, C.M., and Rollins, M.B. (1998). Low-temperature carbon utilization is regulated by novel gene activity in the heart of a hibernating mammal. Proc Natl Acad Sci U S A 95, 8392-8397.

Andrews, M.T., Russeth, K.P., Drewes, L.R., and Henry, P.-G. (2009). Adaptive mechanisms regulate preferred utilization of ketones in the heart and brain of a hibernating mammal during arousal from torpor. Am J Physiol Regul Integr Comp Physiol 296, R383-R393.

Armstrong, C., and Staples, J.F. (2010). The role of succinate dehydrogenase and oxaloacetate in metabolic suppression during hibernation and arousal. J Comp Physiol B, Biochem Syst Environ Physiol 180, 775-783.

Bannister, A.J., and Kouzarides, T. (2011). Regulation of chromatin by histone modifications. Cell Res 21,381-395.

Barski, A., Cuddapah, S., Cui, K., Roh, T.-Y., Schones, D.E., Wang, Z., Wei, G., Chepelev, I., and Zhao, K. (2007). High-Resolution Profiling of Histone Methylations in the Human Genome. Cell 129, 823-837.

Bartke, T., Vermeulen, M., Xhemalce, B., Robson, S.C., Mann, M., and Kouzarides, T. (2010). Nucleosome-interacting proteins regulated by DNA and histone 
methylation. Cell 143, 470-484.

Bauman, W.A., Meryn, S., and Florant, G.L. (1987). Pancreatic hormones in the nonhibernating and hibernating golden mantled ground squirrel. Comp Biochem Physiol a Physiol 86, 241-244.

Beck, D.B., Oda, H., Shen, S.S., and Reinberg, D. (2012). PR-Set7 and H4K20me1: at the crossroads of genome integrity, cell cycle, chromosome condensation, and transcription. Genes Dev. 26, 325-337.

Bell, R.A.V., Smith, J.C., and Storey, K.B. (2014). Purification and properties of glyceraldehyde-3-phosphate dehydrogenase from the skeletal muscle of the hibernating ground squirrel, Ictidomys tridecemlineatus. PeerJ 2, e634.

Berger, S.L. (2002). Histone modifications in transcriptional regulation. Curr Opin Genet Dev 12, 142-148.

Bernstein, B.E., Meissner, A., and Lander, E.S. (2007). The mammalian epigenome. Cell $128,669-681$.

Biggar, K.K., and Li, S.S.C. (2015). Non-histone protein methylation as a regulator of cellular signalling and function. Nat Rev Mol Cell Biol 16, 5-17.

Biggar, K.K., Wu, C.-W., Tessier, S.N., Zhang, J., Pifferi, F., Perret, M., and Storey, K.B. (2015). Modulation of Gene Expression in Key Survival Pathways During Daily Torpor in the Gray Mouse Lemur, Microcebus murinus. Genomics Proteomics Bioinformatics 13, 111-118.

Biggar, Y., and Storey, K.B. (2014). Global DNA modifications suppress transcription in brown adipose tissue during hibernation. Cryobiology 69, 333-338.

Binda, O. (2013). On your histone mark, SET, methylate! Epigenetics 8, 457-463.

Binda, O., LeRoy, G., Bua, D.J., Garcia, B.A., Gozani, O., and Richard, S. (2010). Trimethylation of histone $\mathrm{H} 3$ lysine 4 impairs methylation of histone $\mathrm{H} 3$ lysine 9: regulation of lysine methyltransferases by physical interaction with their substrates. Epigenetics 5, 767-775.

Boccuni, P., MacGrogan, D., Scandura, J.M., and Nimer, S.D. (2003). The human L(3)MBT polycomb group protein is a transcriptional repressor and interacts physically and functionally with TEL (ETV6). J Biol Chem 278, 15412-15420.

Botuyan, M.V., Lee, J., Ward, I.M., Kim, J.-E., Thompson, J.R., Chen, J., and Mer, G. (2006). Structural Basis for the Methylation State-Specific Recognition of Histone H4-K20 by 53BP1 and Crb2 in DNA Repair. Cell 127, 1361-1373.

Boyer, B.B., Barnes, B.M., Kopecky, J., and Jacobsson, A. (1993). Molecular control of prehibernation brown fat growth in arctic ground squirrels. In Life in the Cold III: 
Ecological, Physiological and Molecular Mechanisms, G.L. Florant, B.A. Wunder, B. Horwitz, and C. Carey, eds. (Boulder, CO: Westview Press), pp. 483-491.

Boyer, B.B., and Barnes, B.M. (1999). Molecular and Metabolic Aspects of Mammalian Hibernation. BioScience 49, 713-724.

Börsch-Haubold, A.G., Montero, I., Konrad, K., and Haubold, B. (2014). Genome-wide quantitative analysis of histone $\mathrm{H} 3$ lysine 4 trimethylation in wild house mouse liver: environmental change causes epigenetic plasticity. PLoS ONE 9, e97568.

Brooks, N.E., Myburgh, K.H., and Storey, K.B. (2011). Myostatin levels in skeletal muscle of hibernating ground squirrels. J Exp Biol 214, 2522-2527.

Brooks, N.E., Myburgh, K.H., and Storey, K.B. (2015). Muscle satellite cells increase during hibernation in ground squirrels. Comp Biochem Physiol B, Biochem Mol Biol 189, 55-61.

Brooks, S.P.J., and Storey, K.B. (1992). Mechanisms of glycolytic control during hibernation in the ground squirrel Spermophilus lateralis. J Comp Physiol B 162, $23-28$.

Brown, J.C.L., Chung, D.J., Belgrave, K.R., and Staples, J.F. (2012). Mitochondrial metabolic suppression and reactive oxygen species production in liver and skeletal muscle of hibernating thirteen-lined ground squirrels. Am J Physiol Regul Integr Comp Physiol 302, R15-R28.

Brown, J.C.L., Chung, D.J., Cooper, A.N., and Staples, J.F. (2013). Regulation of succinate-fuelled mitochondrial respiration in liver and skeletal muscle of hibernating thirteen-lined ground squirrels. J Exp Biol 216, 1736-1743.

Buck, C.L., and Barnes, B.M. (1999). Annual Cycle of Body Composition and Hibernation in Free-Living Arctic Ground Squirrels. J Mammal 80, 430-442.

Buck, M.J., Squire, T.L., and Andrews, M.T. (2002). Coordinate expression of the PDK4 gene: a means of regulating fuel selection in a hibernating mammal. Physiol Genomics 8, 5-13.

Cain, J.A., Solis, N., and Cordwell, S.J. (2014). Beyond gene expression: the impact of protein post-translational modifications in bacteria. J Proteomics 97, 265-286.

Calpena, E., Palau, F., Espinós, C., and Galindo, M.I. (2015). Evolutionary History of the Smyd Gene Family in Metazoans: A Framework to Identify the Orthologs of Human Smyd Genes in Drosophila and Other Animal Species. PLoS ONE 10, e0134106.

Cao, D.S., Wang, Z.G., Zhang, C.L., Oh, J., Xing, W.B., Li, S.J., Richardson, J.A., Wang, D.Z., and Olson, E.N. (2005). Modulation of smooth muscle gene expression by association of histone acetyltransferases and deacetylases with 
myocardin. Mol. Cell. Biol. 25, 364-376.

Cao, F., Chen, Y., Cierpicki, T., Liu, Y., Basrur, V., Lei, M., and Dou, Y. (2010). An Ash2L/RbBP5 heterodimer stimulates the MLL1 methyltransferase activity through coordinated substrate interactions with the MLL1 SET domain. PLoS ONE 5, e14102.

Carey, H.V., Andrews, M.T., and Martin, S.L. (2003). Mammalian hibernation: Cellular and molecular responses to depressed metabolism and low temperature. Physiol $\operatorname{Rev} 83,1153-1181$.

Carey, H.V., Sills, N.S., and Gorham, D.A. (1999). Stress proteins in mammalian hibernation. Am Zool 39, 825-835.

Carr, S.M., Munro, S., and La Thangue, N.B. (2012). Lysine methylation and the regulation of p53. Essays Biochem 52, 79-92.

Chaturvedi, C.-P., Somasundaram, B., Singh, K., Carpenedo, R.L., Stanford, W.L., Dilworth, F.J., and Brand, M. (2012). Maintenance of gene silencing by the coordinate action of the H3K9 methyltransferase G9a/KMT1C and the H3K4 demethylase Jarid1a/KDM5A. Proc Natl Acad Sci U.S.a. 109, 18845-18850.

Chen, T., and Dent, S.Y.R. (2014). Chromatin modifiers and remodellers: regulators of cellular differentiation. Nature Publishing Group 15, 93-106.

Cheng, J., Blum, R., Bowman, C., Hu, D., Shilatifard, A., Shen, S., and Dynlacht, B.D. (2014). A role for H3K4 monomethylation in gene repression and partitioning of chromatin readers. Mol Cell 53, 979-992.

Chew, Y.C., Camporeale, G., Kothapalli, N., Sarath, G., and Zempleni, J. (2006). Lysine residues in $\mathrm{N}$-terminal and $\mathrm{C}$-terminal regions of human histone $\mathrm{H} 2 \mathrm{~A}$ are targets for biotinylation by biotinidase. J. Nutr. Biochem. 17, 225-233.

Chin, H.G., Estève, P.-O., Pradhan, M., Benner, J., Patnaik, D., Carey, M.F., and Pradhan, S. (2007). Automethylation of G9a and its implication in wider substrate specificity and HP1 binding. Nucleic Acids Res 35, 7313-7323.

Chin, H.G., Patnaik, D., Estève, P.-O., Jacobsen, S.E., and Pradhan, S. (2006). Catalytic properties and kinetic mechanism of human recombinant Lys-9 histone $\mathrm{H} 3$ methyltransferase SUV39H1: participation of the chromodomain in enzymatic catalysis. Biochemistry 45, 3272-3284.

Cho, H.-S., Shimazu, T., Toyokawa, G., Daigo, Y., Maehara, Y., Hayami, S., Ito, A., Masuda, K., Ikawa, N., Field, H.I., et al. (2012). Enhanced HSP70 lysine methylation promotes proliferation of cancer cells through activation of Aurora kinase B. Nat Commun 3, 1072.

Cho, H.-S., Suzuki, T., Dohmae, N., Hayami, S., Unoki, M., Yoshimatsu, M., Toyokawa, 
G., Takawa, M., Chen, T., Kurash, J.K., et al. (2011). Demethylation of RB regulator MYPT1 by histone demethylase LSD1 promotes cell cycle progression in cancer cells. Cancer Res 71, 655-660.

Chuikov, S., Kurash, J.K., Wilson, J.R., Xiao, B., Justin, N., Ivanov, G.S., McKinney, K., Tempst, P., Prives, C., Gamblin, S.J., et al. (2004). Regulation of p53 activity through lysine methylation. Nature 432, 353-360.

Claycombe, K.J., Vomhof-DeKrey, E.E., Garcia, R., Johnson, W.T., Uthus, E., and Roemmich, J.N. (2016). Decreased beige adipocyte number and mitochondrial respiration coincide with increased histone methyl transferase (G9a) and reduced FGF21 gene expression in Sprague-Dawley rats fed prenatal low protein and postnatal high-fat diets. J. Nutr. Biochem. 31, 113-121.

Cotton, C.J. (2016). Skeletal muscle mass and composition during mammalian hibernation. J Exp Biol 219, 226-234.

Cui, G., Park, S., Badeaux, A.I., Kim, D., Lee, J., Thompson, J.R., Yan, F., Kaneko, S., Yuan, Z., Botuyan, M.V., et al. (2012). PHF20 is an effector protein of p53 double lysine methylation that stabilizes and activates p53. Nat Struct Mol Biol 19, 916924.

Dang, K., Li, Y.-Z., Gong, L.-C., Xue, W., Wang, H.-P., Goswami, N., and Gao, Y.-F. (2016). Stable atrogin-1 (Fbxo32) and MuRF1 (Trim63) gene expression is involved in the protective mechanism in soleus muscle of hibernating Daurian ground squirrels (Spermophilus dauricus). Biol Open 5, 62-71.

Dark, J., and Miller, D.R. (1997). Metabolic fuel privation in hibernating and awake ground squirrels. Physiol Behav 63, 59-65.

Dark, J. (2005). Annual lipid cycles in hibernators: integration of physiology and behavior. Annu Rev Nutr 25, 469-497.

Del Rizzo, P.A., and Trievel, R.C. (2011). Substrate and product specificities of SET domain methyltransferases. 6, 1059-1067.

Dhayalan, A., Kudithipudi, S., Rathert, P., and Jeltsch, A. (2011). Specificity AnalysisBased Identification of New Methylation Targets of the SET7/9 Protein Lysine Methyltransferase. Chemistry \& Biology 18, 111-120.

Donlin, L.T., Andresen, C., Just, S., Rudensky, E., Pappas, C.T., Kruger, M., Jacobs, E.Y., Unger, A., Zieseniss, A., Dobenecker, M.-W., et al. (2012). Smyd2 controls cytoplasmic lysine methylation of Hsp90 and myofilament organization. Genes Dev 26, 114-119.

Dou, Y.L., Milne, T.A., Tackett, A.J., Smith, E.R., Fukuda, A., Wysocka, J., Allis, C.D., Chait, B.T., Hess, J.L., and Roeder, R.G. (2005). Physical association and coordinate function of the H3K4 methyltransferase MLL1 and the H4K16 
acetyltransferase MOF. Cell 121, 873-885.

Drew, K.L., Buck, C.L., Barnes, B.M., Christian, S.L., Rasley, B.T., and Harris, M.B. (2007). Central nervous system regulation of mammalian hibernation: implications for metabolic suppression and ischemia tolerance. J Neurochem 102, 1713-1726.

Du, S.J., Tan, X., and Zhang, J. (2014). SMYD proteins: key regulators in skeletal and cardiac muscle development and function. Anat Rec (Hoboken) 297, 1650-1662.

Duan, G., and Walther, D. (2015). The roles of post-translational modifications in the context of protein interaction networks. PLoS Comput Biol 11, e1004049.

E, Q., Liu, X., Liu, Y., Liu, W., and Zuo, J. (2013). Over-expression of GRP75 inhibits liver injury induced by oxidative damage. Acta Biochim Biophys Sin 45, 129-134.

Eaton, S.L., Roche, S.L., Llavero Hurtado, M., Oldknow, K.J., Farquharson, C., Gillingwater, T.H., and Wishart, T.M. (2013). Total protein analysis as a reliable loading control for quantitative fluorescent Western blotting. 8, e72457.

Enwere, E.K., Lacasse, E.C., Adam, N.J., and Korneluk, R.G. (2014). Role of the TWEAK-Fn14-cIAP1-NF- $\kappa$ B Signaling Axis in the Regulation of Myogenesis and Muscle Homeostasis. Front Immunol 5, 34.

Epperson, L.E., Dahl, T.A., and Martin, S.L. (2004). Quantitative analysis of liver protein expression during hibernation in the golden-mantled ground squirrel. Mol Cell Proteomics 3, 920-933.

Epperson, L.E., Rose, J.C., Carey, H.V., and Martin, S.L. (2010a). Seasonal proteomic changes reveal molecular adaptations to preserve and replenish liver proteins during ground squirrel hibernation. Am J Physiol Regul Integr Comp Physiol 298, R329-R340.

Epperson, L.E., Rose, J.C., Russell, R.L., Nikrad, M.P., Carey, H.V., and Martin, S.L. (2010b). Seasonal protein changes support rapid energy production in hibernator brainstem. J Comp Physiol B 180, 599-617.

Ernst, J., Kheradpour, P., Mikkelsen, T.S., Shoresh, N., Ward, L.D., Epstein, C.B., Zhang, X., Wang, L., Issner, R., Coyne, M., et al. (2011). Mapping and analysis of chromatin state dynamics in nine human cell types. Nature 473, 43-49.

Ernst, P., and Vakoc, C.R. (2012). WRAD: enabler of the SET1-family of H3K4 methyltransferases. Brief Funct Genomics 11, 217-226.

Estève, P.-O., Chin, H.G., Smallwood, A., Feehery, G.R., Gangisetty, O., Karpf, A.R., Carey, M.F., and Pradhan, S. (2006). Direct interaction between DNMT1 and G9a coordinates DNA and histone methylation during replication. Genes Dev 20, 3089 3103. 
Fahlman, A., Storey, J.M., and Storey, K.B. (2000). Gene Up-Regulation in Heart during Mammalian Hibernation. Cryobiology 40, 332-342.

Falkenberg, K.J., and Johnstone, R.W. (2014). Histone deacetylases and their inhibitors in cancer, neurological diseases and immune disorders. Nat Rev Drug Discov 13, 673-691.

Fan, J., Krautkramer, K.A., Feldman, J.L., and Denu, J.M. (2015). Metabolic Regulation of Histone Post-Translational Modifications. ACS Chem. Biol. 10, 95-108.

Feder, M.E., and Hofmann, G.E. (1999). Heat-shock proteins, molecular chaperones, and the stress response: evolutionary and ecological physiology. Annu Rev Physiol 61, 243-282.

Fedorov, V.B., Goropashnaya, A.V., Stewart, N.C., Tøien, Ø., Chang, C., Wang, H., Yan, J., Showe, L.C., Showe, M.K., and Barnes, B.M. (2014). Comparative functional genomics of adaptation to muscular disuse in hibernating mammals. Mol Ecol 23, $5524-5537$.

Fleck, C.C., and Carey, H.V. (2005). Modulation of apoptotic pathways in intestinal mucosa during hibernation. Am J Physiol Regul Integr Comp Physiol 289, R586R595.

Florant, G.L., and Healy, J.E. (2012). The regulation of food intake in mammalian hibernators: a review. J Comp Physiol B, Biochem Syst Environ Physiol 182, 451467.

Florant, G.L., Fenn, A.M., Healy, J.E., Wilkerson, G.K., and Handa, R.J. (2010). To eat or not to eat: the effect of AICAR on food intake regulation in yellow-bellied marmots (Marmota flaviventris). J Exp Biol 213, 2031-2037.

Florant, G.L., Nuttle, L.C., Mullinex, D.E., and Rintoul, D.A. (1990). Plasma and white adipose tissue lipid composition in marmots. Am J Physiol 258, R1123-R1131.

Fnu, S., Williamson, E.A., De Haro, L.P., Brenneman, M., Wray, J., Shaheen, M., Radhakrishnan, K., Lee, S.-H., Nickoloff, J.A., and Hromas, R. (2011). Methylation of histone H3 lysine 36 enhances DNA repair by nonhomologous endjoining. Proc Natl Acad Sci U.S.a. 108, 540-545.

Fons, R., Sender, S., Peters, T., and Jurgens, K.D. (1997). Rates of rewarming, heart and respiratory rates and their significance for oxygen transport during arousal from torpor in the smallest mammal, the Etruscan shrew Suncus etruscus. J Exp Biol 200, 1451-1458.

Foster, D.O., and Frydman, M.L. (1979). Tissue distribution of cold-induced thermogenesis in conscious warm- or cold-acclimated rats reevaluated from changes in tissue blood flow: The dominant role of brown adipose tissue in the replacement of shivering by nonshivering thermogenesis. Can J Physiol Pharmacol 
$57,257-270$.

Frayn, K.N., and Arner, P. (2006). Fatty acid metabolism in adipose tissue, muscle and liver in health and disease. Essays Biochem.

French, A.R. (1985). Allometries of the durations of torpid and euthermic intervals during mammalian hibernation: A test of the theory of metabolic control of the timing of changes in body temperature. J Comp Physiol B 156, 13-19.

Frerichs, K.U., Kennedy, C., Sokoloff, L., and Hallenbeck, J.M. (1994). Local cerebral blood flow during hibernation, a model of natural tolerance to "cerebral ischemia". J Cereb Blood Flow Metab 14, 193-205.

Frerichs, K.U., Smith, C.B., Brenner, M., DeGracia, D.J., Krause, G.S., Marrone, L., Dever, T.E., and Hallenbeck, J.M. (1998). Suppression of protein synthesis in brain during hibernation involves inhibition of protein initiation and elongation. Proc Natl Acad Sci U S A 95, 14511-14516.

Fujita, N., Watanabe, S., Ichimura, T., Tsuruzoe, S., Shinkai, Y., Tachibana, M., Chiba, T., and Nakao, M. (2003). Methyl-CpG Binding Domain 1 (MBD1) Interacts with the Suv39h1-HP1 Heterochromatic Complex for DNA Methylation-based Transcriptional Repression. J Biol Chem 278, 24132-24138.

Galster, W., and Morrison, P.R. (1975). Gluconeogenesis in arctic ground squirrels between periods of hibernation. Am J Physiol 228, 325-330.

Gamsjaeger, R., Webb, S.R., Lamonica, J.M., Billin, A., Blobel, G.A., and Mackay, J.P. (2011). Structural basis and specificity of acetylated transcription factor GATA1 recognition by BET family bromodomain protein Brd3. Mol Cell Biol 31, 26322640 .

Garapaty, S., Xu, C.-F., Trojer, P., Mahajan, M.A., Neubert, T.A., and Samuels, H.H. (2009). Identification and Characterization of a Novel Nuclear Protein Complex Involved in Nuclear Hormone Receptor-mediated Gene Regulation. J Biol Chem $284,7542-7552$.

Garcia-Bassets, I., Kwon, Y.-S., Telese, F., Prefontaine, G.G., Hutt, K.R., Cheng, C.S., Ju, B.-G., Ohgi, K.A., Wang, J., Escoubet-Lozach, L., et al. (2007). Histone methylation-dependent mechanisms impose ligand dependency for gene activation by nuclear receptors. Cell 128, 505-518.

Gehnrich, S.C., and Aprille, J.R. (1988). Hepatic gluconeogenesis and mitochondrial function during hibernation. Comp. Biochem. Physiol., B 91, 11-16.

Gervin, C.M., McCulla, A., Williams, M., and Ouhtit, A. (2003). Dysfunction of p53 in photocarcinogenesis. Front Biosci 8, s715-s717.

Gu, B., and Lee, M.G. (2013). Histone H3 lysine 4 methyltransferases and demethylases 
in self-renewal and differentiation of stem cells. Cell Biosci 3, 39.

Guo, H.-B., and Guo, H. (2007). Mechanism of histone methylation catalyzed by protein lysine methyltransferase SET7/9 and origin of product specificity. Proc Natl Acad Sci U S A 104, 8797-8802.

Hamamoto, R., Saloura, V., and Nakamura, Y. (2015a). Critical roles of non-histone protein lysine methylation in human tumorigenesis. Nat. Rev. Cancer 15, 110-124.

Hamamoto, R., Saloura, V., and Nakamura, Y. (2015b). Critical roles of non-histone protein lysine methylation in human tumorigenesis. Nat. Rev. Cancer 15, 110-124.

Haman, F. (2006). Shivering in the cold: from mechanisms of fuel selection to survival. J Appl Physiol 100, 1702-1708.

Hampton, M., and Andrews, M.T. (2007). A simple molecular mathematical model of mammalian hibernation. J Theor Biol 247, 297-302.

Hampton, M., Melvin, R.G., Kendall, A.H., Kirkpatrick, B.R., Peterson, N., and Andrews, M.T. (2011). Deep sequencing the transcriptome reveals seasonal adaptive mechanisms in a hibernating mammal. PLoS ONE 6, e27021.

Hardie, D.G., Hawley, S.A., and Scott, J.W. (2006). AMP-activated protein kinase development of the energy sensor concept. J Physiol 574, 7-15.

He, A., Shen, X., Ma, Q., Cao, J., Gise, von, A., Zhou, P., Wang, G., Marquez, V.E., Orkin, S.H., and Pu, W.T. (2012). PRC2 directly methylates GATA4 and represses its transcriptional activity. Genes Dev. 26, 37-42.

Healy, J.E., and Florant, G.L. (2012). Ghrelin, Leptin, and Fatty Acids in Free-Living Callospermophilus lateralis (Golden-Mantled Ground Squirrels). In Living in a Seasonal World, T. Ruf, C. Bieber, W. Arnold, and E. Millesi, eds. (Berlin, Heidelberg: Springer Berlin Heidelberg), pp. 519-529.

Healy, J.E., Bateman, J.L., Ostrom, C.E., and Florant, G.L. (2011a). Peripheral ghrelin stimulates feeding behavior and positive energy balance in a sciurid hibernator. Horm Behav 59, 512-519.

Healy, J.E., Gearhart, C.N., Bateman, J.L., Handa, R.J., and Florant, G.L. (2011b). AMPK and ACCchange with fasting and physiological condition in euthermic and hibernating golden-mantled ground squirrels (Callospermophilus lateralis). Comp Biochem Physiol a Physiol 159, 322-331.

Healy, J.E., Ostrom, C.E., Wilkerson, G.K., and Florant, G.L. (2010). Plasma ghrelin concentrations change with physiological state in a sciurid hibernator (Spermophilus lateralis). Gen Comp Endocrinol 166, 372-378.

Heard, E. (2005). Delving into the diversity of facultative heterochromatin: the 
epigenetics of the inactive X chromosome. Curr Opin Genet Dev 15, 482-489.

Hefler, J., Wu, C.-W., and Storey, K.B. (2015). Transcriptional Activation of p53 during Cold Induced Torpor in the 13-Lined Ground Squirrel Ictidomys tridecemlineatus. Biochem Res Int 2015, 731595-11.

Heldmaier, G., Ortmann, S., and Elvert, R. (2004). Natural hypometabolism during hibernation and daily torpor in mammals. Respir Physiol Neurobiol 141, 317-329.

Hindle, A.G., Grabek, K.R., Epperson, L.E., Karimpour-Fard, A., and Martin, S.L. (2014). Metabolic changes associated with the long winter fast dominate the liver proteome in 13-lined ground squirrels. Physiol Genomics 46, 348-361.

Hindle, A.G., Karimpour-Fard, A., Epperson, L.E., Hunter, L.E., and Martin, S.L. (2011). Skeletal muscle proteomics: carbohydrate metabolism oscillates with seasonal and torpor-arousal physiology of hibernation. Am J Physiol Regul Integr Comp Physiol 301, R1440-R1452.

Hindle, A.G., Otis, J.P., Epperson, L.E., Hornberger, T.A., Goodman, C.A., Carey, H.V., and Martin, S.L. (2015). Prioritization of skeletal muscle growth for emergence from hibernation. J Exp Biol 218, 276-284.

Hittel, D., and Storey, K.B. (2001). Differential expression of adipose-and heart-type fatty acid binding proteins in hibernating ground squirrels. BBA-Gene Struct Expr $1522,238-243$.

Hittel, D., and Storey, K.B. (2002). The translation state of differentially expressed mRNAs in the hibernating 13-lined ground squirrel (Spermophilus tridecemlineatus). Arch Biochem Biophys 401, 244-254.

Hoo-Paris, R., Castex, C., Hamsany, M., Thari, A., and Sutter, B. (1985). GlucagonSecretion in the Hibernating Edible Dormouse (Glis-Glis). Comp Biochem Physiol a Physiol 81, 277-281.

Hsia, D.A., Tepper, C.G., Pochampalli, M.R., Hsia, E.Y.C., Izumiya, C., Huerta, S.B., Wright, M.E., Chen, H.-W., Kung, H.-J., and Izumiya, Y. (2010). KDM8, a H3K36me2 histone demethylase that acts in the cyclin A1 coding region to regulate cancer cell proliferation. Proc Natl Acad Sci U.S.a. 107, 9671-9676.

Huang, J., Dorsey, J., Chuikov, S., Zhang, X., Jenuwein, T., Reinberg, D., and Berger, S.L. (2010). G9a and Glp Methylate Lysine 373 in the Tumor Suppressor p53. J. Biol. Chem. 285, 9636-9641.

Huang, J., Perez-Burgos, L., Placek, B.J., Sengupta, R., Richter, M., Dorsey, J.A., Kubicek, S., Opravil, S., Jenuwein, T., and Berger, S.L. (2006). Repression of p53 activity by Smyd2-mediated methylation. Nature 444, 629-632.

Huang, J., Sengupta, R., Espejo, A.B., Lee, M.G., Dorsey, J.A., Richter, M., Opravil, S., 
Shiekhattar, R., Bedford, M.T., Jenuwein, T., et al. (2007). p53 is regulated by the lysine demethylase LSD1. Nature 449, 105-108.

Huang, P.-H., Plass, C., and Chen, C.-S. (2011). Effects of Histone Deacetylase Inhibitors on Modulating H3K4 Methylation Marks - A Novel Cross-Talk Mechanism between Histone-Modifying Enzymes. Mol Cell Pharmacol 3, 39-43.

Humphries, M.M., Thomas, D.W., and Kramer, D.L. (2003). The role of energy availability in Mammalian hibernation: a cost-benefit approach. Physiol Biochem Zool 76, 165-179.

Ivakine, E.A., and Cohn, R.D. (2014). Maintaining skeletal muscle mass: lessons learned from hibernation. Exp. Physiol. 99, 632-637.

Ivanov, G.S., Ivanova, T., Kurash, J., Ivanov, A., Chuikov, S., Gizatullin, F., HerreraMedina, E.M., Rauscher, F., Reinberg, D., and Barlev, N.A. (2007). Methylationacetylation interplay activates p53 in response to DNA damage. Mol. Cell. Biol. 27, 6756-6769.

Izzo, A., and Schneider, R. (2010). Chatting histone modifications in mammals. Brief Funct Genomics 9, 429-443.

Jayaraman, S. (2012). Epigenetic mechanisms of metabolic memory in diabetes. Circulation Research 110, 1039-1041.

Jenuwein, T. (2006). The epigenetic magic of histone lysine methylation. FEBS Journal 273, 3121-3135.

Johnstone, S.E., and Baylin, S.B. (2010). Stress and the epigenetic landscape: a link to the pathobiology of human diseases? Nature Publishing Group 11, 806-812.

Jun, H.-J., Kim, J., Hoang, M.-H., and Lee, S.-J. (2012). Hepatic lipid accumulation alters global histone h3 lysine 9 and 4 trimethylation in the peroxisome proliferatoractivated receptor alpha network. PLoS ONE 7, e44345.

Jørgensen, S., Schotta, G., and Sørensen, C.S. (2013). Histone H4 lysine 20 methylation: key player in epigenetic regulation of genomic integrity. Nucleic Acids Res 41, 2797-2806.

Karpovich, S.A., Tøien, Ø., Buck, C.L., and Barnes, B.M. (2009). Energetics of arousal episodes in hibernating arctic ground squirrels. J Comp Physiol B, Biochem Syst Environ Physiol 179, 691-700.

Keating, S.T., and El-Osta, A. (2013a). Epigenetic changes in diabetes. Clin Genet 84, $1-$ 10.

Keating, S.T., and El-Osta, A. (2013b). Transcriptional regulation by the Set7 lysine methyltransferase. Epigenetics 8, 361-372. 
Keating, S.T., and El-Osta, A. (2015). Epigenetics and metabolism. Circulation Research $116,715-736$.

Khu, L.Y., Storey, K.B., Rubtsov, A.M., and Goncharova, N.Y. (2014). Regulation of glucokinase activity in liver of hibernating ground squirrel Spermophilus undulatus. Biochemistry Mosc. 79, 727-732.

Khurana, N., and Bhattacharyya, S. (2015). Hsp90, the concertmaster: tuning transcription. Front Oncol 5, 100.

Kim, Y., Nam, H.J., Lee, J., Park, D.Y., Kim, C., Yu, Y.S., Kim, D., Park, S.W., Bhin, J., Hwang, D., et al. (2016). Methylation-dependent regulation of HIF-1 $\alpha$ stability restricts retinal and tumour angiogenesis. Nat Commun 7, 10347.

Kirchner, H., Osler, M.E., Krook, A., and Zierath, J.R. (2013). Epigenetic flexibility in metabolic regulation: disease cause and prevention? Trends Cell Biol. 23, 203-209.

Kisser, B., and Goodwin, H.T. (2012). Hibernation and Overwinter Body Temperatures in Free-Ranging Thirteen-Lined Ground Squirrels, Ictidomys tridecemlineatus. Am Midl Nat 167, 396-409.

Klain, G.J., and Whitten, B.K. (1968). Plasma free amino acids in hibernation and arousal. Comp Biochem Physiol 27, 617-619.

Kloner, R.A., Bolli, R., Marban, E., Reinlib, L., Braunwald, E., Reinlib, L.J., Bonow, R.O., Borgers, M., Downey, J., Feigenbaum, H., et al. (1998). Medical and cellular implications of stunning, hibernation, and preconditioning - An NHLBI Workshop. Circulation 97, 1848-1867.

Klug, B.J., and Brigham, R.M. (2015). Changes to Metabolism and Cell Physiology that Enable Mammalian Hibernation. Springer Science Reviews 3, 39-56.

Knight, J.E., Narus, E.N., Martin, S.L., Jacobson, A., Barnes, B.M., and Boyer, B.B. (2000). mRNA Stability and Polysome Loss in Hibernating Arctic Ground Squirrels (Spermophilus parryii). Mol. Cell. Biol. 20, 6374-6379.

Kouzarides, T. (2007). Chromatin modifications and their function. Cell 128, 693-705.

Kötter, S., Unger, A., Hamdani, N., Lang, P., Vorgerd, M., Nagel-Steger, L., and Linke, W.A. (2014). Human myocytes are protected from titin aggregation-induced stiffening by small heat shock proteins. J Cell Biol 204, 187-202.

Krivoruchko, A., and Storey, K.B. (2015). Turtle anoxia tolerance: Biochemistry and gene regulation. Biochim Biophys Acta 1850, 1188-1196.

Lan, F., and Shi, Y. (2009). Epigenetic regulation: methylation of histone and nonhistone proteins. Sci China, C, Life Sci 52, 311-322. 
Lau, J.C.Y., Hanel, M.L., and Wevrick, R. (2004). Tissue-specific and imprinted epigenetic modifications of the human NDN gene. Nucleic Acids Res. 32, 33763382.

Lee, K., Park, J.Y., Yoo, W., Gwag, T., Lee, J.-W., Byun, M.-W., and Choi, I. (2008). Overcoming muscle atrophy in a hibernating mammal despite prolonged disuse in dormancy: proteomic and molecular assessment. J Cell Biochem 104, 642-656.

Lee, S., Roeder, R.G., and Lee, J.W. (2009). Roles of Histone H3-Lysine 4 Methyltransferase Complexes in NR-Mediated Gene Transcription. Prog Mol Biol Transl Sci 87, 343-382.

Leung, A., Parks, B.W., Du, J., Trac, C., Setten, R., Chen, Y., Brown, K., Lusis, A.J., Natarajan, R., and Schones, D.E. (2014). Open chromatin profiling in mice livers reveals unique chromatin variations induced by high fat diet. J. Biol. Chem. 289, 23557-23567.

Li, B., Gogol, M., Carey, M., Pattenden, S.G., Seidel, C., and Workman, J.L. (2007). Infrequently transcribed long genes depend on the Set $2 / \mathrm{Rpd} 3 \mathrm{~S}$ pathway for accurate transcription. Genes Dev 21, 1422-1430.

Li, B., Jackson, J., Simon, M.D., Fleharty, B., Gogol, M., Seidel, C., Workman, J.L., and Shilatifard, A. (2009). Histone H3 lysine 36 dimethylation (H3K36me2) is sufficient to recruit the Rpd3s histone deacetylase complex and to repress spurious transcription. J Biol Chem 284, 7970-7976.

Li, Y., Reddy, M.A., Miao, F., Shanmugam, N., Yee, J.-K., Hawkins, D., Ren, B., and Natarajan, R. (2008). Role of the histone H3 lysine 4 methyltransferase, SET7/9, in the regulation of NF-kappaB-dependent inflammatory genes. Relevance to diabetes and inflammation. J Biol Chem 283, 26771-26781.

Liang, G., Lin, J.C.Y., Wei, V., Yoo, C., Cheng, J.C., Nguyen, C.T., Weisenberger, D.J., Egger, G., Takai, D., Gonzales, F.A., et al. (2004). Distinct localization of histone $\mathrm{H} 3$ acetylation and $\mathrm{H} 3-\mathrm{K} 4$ methylation to the transcription start sites in the human genome. Proc Natl Acad Sci U S A 101, 7357-7362.

Liu, L., Cheung, T.H., Charville, G.W., Hurgo, B.M.C., Leavitt, T., Shih, J., Brunet, A., and Rando, T.A. (2013). Chromatin modifications as determinants of muscle stem cell quiescence and chronological aging. Cell Rep 4, 189-204.

Logan, S.M., Luu, B.E., and Storey, K.B. (2016a). Turn down genes for WAT? Activation of anti-apoptosis pathways protects white adipose tissue in metabolically depressed thirteen-lined ground squirrels. Mol Cell Biochem 416, 47-62.

Logan, S.M., Tessier, S.N., Tye, J., and Storey, K.B. (2016b). Response of the JAKSTAT pathway to mammalian hibernation in 13-lined ground squirrel striated muscle. Mol Cell Biochem 414, 115-127. 
Lovegrove, B.G., Körtner, G., and Geiser, F. (1999). The energetic cost of arousal from torpor in the marsupial Sminthopsis macroura: benefits of summer ambient temperature cycles. J Comp Physiol B 169, 11-18.

Luger, K., Mäder, A.W., Richmond, R.K., Sargent, D.F., and Richmond, T.J. (1997). Crystal structure of the nucleosome core particle at $2.8 \AA$ Aesolution. Nature 389, 251-260.

MacDonald, J.A., and Storey, K.B. (1999). Regulation of ground squirrel $\mathrm{Na}+\mathrm{K}+-$ ATPase activity by reversible phosphorylation during hibernation. Biochem Biophys Res Commun 254, 424-429.

Mal, A., Sturniolo, M., Schiltz, R.L., Ghosh, M.K., and Harter, M.L. (2001). A role for histone deacetylase HDAC1 in modulating the transcriptional activity of MyoD: inhibition of the myogenic program. Embo J 20, 1739-1753.

Malatesta, M., Perdoni, F., Battistelli, S., Muller, S., and Zancanaro, C. (2009). The cell nuclei of skeletal muscle cells are transcriptionally active in hibernating edible dormice. BMC Cell Biol. 10, 19.

Manzur, K.L., and Zhou, M.-M. (2005). An archaeal SET domain protein exhibits distinct lysine methyltransferase activity towards DNA-associated protein MC1alpha. FEBS Lett. 579, 3859-3865.

Marengere, L.E., Songyang, Z., Gish, G.D., Schaller, M.D., Parsons, J.T., Stern, M.J., Cantley, L.C., and Pawson, T. (1994). SH2 domain specificity and activity modified by a single residue. Nature $369,502-505$.

Mariño-Ramírez, L., Kann, M.G., Shoemaker, B.A., and Landsman, D. (2005). Histone structure and nucleosome stability. Expert Rev Proteomics 2, 719-729.

Martin, C., and Zhang, Y. (2005). The diverse functions of histone lysine methylation. Nat Rev Mol Cell Biol 6, 838-849.

Masuda, K., Yamada, T., and Ishizawa, R. (2013). Role of myoglobin in regulating respiration during muscle contraction. J Phys Fitness Sports Med 2, 9-16.

McKinnell, I.W., Ishibashi, J., Le Grand, F., Punch, V.G.J., Addicks, G.C., Greenblatt, J.F., Dilworth, F.J., and Rudnicki, M.A. (2008). Pax 7 activates myogenic genes by recruitment of a histone methyltransferase complex. Nat. Cell Biol. 10, 77-84.

McMullen, D.C., and Hallenbeck, J.M. (2010). Regulation of Akt during torpor in the hibernating ground squirrel, Ictidomys tridecemlineatus. J Comp Physiol B, Biochem Syst Environ Physiol 180, 927-934.

Meyer, C.W., Blessing, W., and Heldmaier, G. (2012). Ultradian Episodes of Thermogenesis in Mammals: Implications for the Timing of Torpor Entry and Arousal. In Living in a Seasonal World, T. Ruf, W. Arnold, E. Millesi, and C. 
Bieber, eds. (Berlin, Heidelberg: Springer Berlin Heidelberg), pp. 219-229.

Miao, F., Chen, Z., Genuth, S., Paterson, A., Zhang, L., Wu, X., Li, S.M., Cleary, P., Riggs, A., Harlan, D.M., et al. (2014). Evaluating the role of epigenetic histone modifications in the metabolic memory of type 1 diabetes. Diabetes 63, 1748-1762.

Miao, F., Wu, X., Zhang, L., Yuan, Y.-C., Riggs, A.D., and Natarajan, R. (2007). Genome-wide analysis of histone lysine methylation variations caused by diabetic conditions in human monocytes. J Biol Chem 282, 13854-13863.

Montarras, D., L'honoré, A., and Buckingham, M. (2013). Lying low but ready for action: the quiescent muscle satellite cell. FEBS Journal 280, 4036-4050.

Morales, V., and Richard-Foy, H. (2000). Role of histone N-terminal tails and their acetylation in nucleosome dynamics. Mol. Cell. Biol. 20, 7230-7237.

Morin, P.J., and Storey, K.B. (2005). Cloning and expression of hypoxia-inducible factor 1alpha from the hibernating ground squirrel, Spermophilus tridecemlineatus. BBAGene Regul Mech 1729, 32-40.

Morin, P.J., and Storey, K.B. (2006). Evidence for a reduced transcriptional state during hibernation in ground squirrels. Cryobiology 53, 310-318.

Morin, P.J., and Storey, K.B. (2009). Mammalian hibernation: differential gene expression and novel application of epigenetic controls. Int. J. Dev. Biol. 53, 433442.

Morin, P.J., Ni, Z., McMullen, D.C., and Storey, K.B. (2008). Expression of Nrf2 and its downstream gene targets in hibernating 13-lined ground squirrels, Spermophilus tridecemlineatus. Mol Cell Biochem 312, 121-129.

Mosley, A.L., and Ozcan, S. (2003). Glucose regulates insulin gene transcription by hyperacetylation of histone h4. J Biol Chem 278, 19660-19666.

Mostafa, N., Everett, D.C., Chou, S.C., Kong, P.A., Florant, G.L., and Coleman, R.A. (1993). Seasonal changes in critical enzymes of lipogenesis and triacylglycerol synthesis in the marmot (Marmota flaviventris). J Comp Physiol B 163, 463-469.

Muka, T., Nano, J., Voortman, T., Braun, K.V.E., Ligthart, S., Stranges, S., Bramer, W.M., Troup, J., Chowdhury, R., Dehghan, A., et al. (2016). The role of global and regional DNA methylation and histone modifications in glycemic traits and type 2 diabetes: A systematic review. Nutr Metab Cardiovasc Dis 26, 553-566.

Mungamuri, S.K., Wang, S., Manfredi, J.J., Gu, W., and Aaronson, S.A. (2015). Ash2L enables P53-dependent apoptosis by favoring stable transcription pre-initiation complex formation on its pro-apoptotic target promoters. Oncogene 34, 2461-2470.

Mzilikazi, N., Lovegrove, B.G., and Ribble, D.O. (2002). Exogenous passive heating 
during torpor arousal in free-ranging rock elephant shrews, Elephantulus myurus. Oecologia 133, 307-314.

Nedergaard, J., Cannon, B., and Jaenicke, R. (1990). Mammalian Hibernation [and Discussion]. Philos Trans R Soc Lond B Biol Sci 326, 669-686.

Nelson, C.J., Otis, J.P., and Carey, H.V. (2009). A role for nuclear receptors in mammalian hibernation. J Physiol 587, 1863-1870.

Ni, Z., and Storey, K.B. (2010). Heme oxygenase expression and Nrf2 signaling during hibernation in ground squirrels. Can J Physiol Pharmacol 88, 379-387.

Nightingale, K.P., Gendreizig, S., White, D.A., Bradbury, C., Hollfelder, F., and Turner, B.M. (2007). Cross-talk between histone modifications in response to histone deacetylase inhibitors: MLL4 links histone $\mathrm{H} 3$ acetylation and histone H3K4 methylation. J Biol Chem 282, 4408-4416.

Nowell, M.M., Choi, H., and Rourke, B.C. (2011). Muscle plasticity in hibernating ground squirrels (Spermophilus lateralis) is induced by seasonal, but not lowtemperature, mechanisms. J Comp Physiol B 181, 147-164.

O'Hara, B.F., Watson, F.L., Srere, H.K., Kumar, H., Wiler, S.W., Welch, S.K., Bitting, L., Heller, H.C., and Kilduff, T.S. (1999). Gene expression in the brain across the hibernation cycle. J Neurosci 19, 3781-3790.

Okabe, J., Orlowski, C., Balcerczyk, A., Tikellis, C., Thomas, M.C., Cooper, M.E., and El-Osta, A. (2012). Distinguishing hyperglycemic changes by Set7 in vascular endothelial cells. Circulation Research 110, 1067-1076.

Olsen, J.B., Cao, X.-J., Han, B., Chen, L.H., Horvath, A., Richardson, T.I., Campbell, R.M., Garcia, B.A., and Nguyen, H. (2016). Quantitative Profiling of the Activity of Protein Lysine Methyltransferase SMYD2 Using SILAC-Based Proteomics. Mol. Cell Proteomics 15, 892-905.

Otis, J.P., Sahoo, D., Drover, V.A., Yen, C.-L.E., and Carey, H.V. (2011). Cholesterol and lipoprotein dynamics in a hibernating mammal. PLoS ONE 6, e29111.

Özdağ, H., Teschendorff, A.E., Ahmed, A.A., Hyland, S.J., Blenkiron, C., Bobrow, L., Veerakumarasivam, A., Burtt, G., Subkhankulova, T., Arends, M.J., et al. (2006). Differential expression of selected histone modifier genes in human solid cancers. BMC Genomics 7, 90.

Pan, D., Mao, C., Zou, T., Yao, A.Y., Cooper, M.P., Boyartchuk, V., and Wang, Y.-X. (2012). The histone demethylase Jhdmla regulates hepatic gluconeogenesis. PLoS Genet. 8, e1002761.

Pan, P., Treat, M.D., and van Breukelen, F. (2014). A systems-level approach to understanding transcriptional regulation by p53 during mammalian hibernation. $\mathrm{J}$ 
Exp Biol 217, 2489-2498.

Panning, B., and Jaenisch, R. (1998). RNA and the epigenetic regulation of X chromosome inactivation. Cell 93, 305-308.

Patel, A., Dharmarajan, V., Vought, V.E., and Cosgrove, M.S. (2009). On the Mechanism of Multiple Lysine Methylation by the Human Mixed Lineage Leukemia Protein-1 (MLL1) Core Complex. J Biol Chem 284, 24242-24256.

Pałyga, J. (2012). Genetic and epigenetic regulation in hypometabolism. In Hypometabolism: Strategies of Survival in Vertebrates and Invertebrates, M. Caputa, and A. Nowakowska, eds. (Kerala, India: Research Signpost), pp. 183-202.

Peters, A.H.F.M., Mermoud, J.E., O'Carroll, D., Pagani, M., Schweizer, D., Brockdorff, N., and Jenuwein, T. (2002). Histone H3 lysine 9 methylation is an epigenetic imprint of facultative heterochromatin. Nat Genet 30, 77-80.

Pinkney, J. (2014). The role of ghrelin in metabolic regulation. Curr Opin Clin Nutr Metab Care 17, 497-502.

Plath, K., Fang, J., Mlynarczyk-Evans, S.K., Cao, R., Worringer, K.A., Wang, H., la Cruz, de, C.C., Otte, A.P., Panning, B., and Zhang, Y. (2003). Role of histone H3 lysine 27 methylation in X inactivation. Science 300, 131-135.

Postnikova, G.B., Tselikova, S.V., Kolaeva, S.G., and Solomonov, N.G. (1999). Myoglobin content in skeletal muscles of hibernating ground squirrels rises in autumn and winter. Comp Biochem Physiol, Part a Mol Integr Physiol 124, 35-37.

Puri, P.L., Sartorelli, V., Yang, X.J., and Hamamori, Y. (1997). Differential roles of p300 and PCAF acetyltransferases in muscle differentiation. Mol Cell 1, 35-45.

Rao, V.K., Ow, J.R., Shankar, S.R., Bharathy, N., Manikandan, J., Wang, Y., and Taneja, R. (2016). G9a promotes proliferation and inhibits cell cycle exit during myogenic differentiation. Nucleic Acids Res gkw483.

Rathert, P., Dhayalan, A., Murakami, M., Zhang, X., Tamas, R., Jurkowska, R., Komatsu, Y., Shinkai, Y., Cheng, X., and Jeltsch, A. (2008). Protein lysine methyltransferase G9a acts on non-histone targets. Nature Chemical Biology 4, 344-346.

Rea, S., Eisenhaber, F., O'Carroll, N., Strahl, B.D., Sun, Z.W., Schmid, M., Opravil, S., Mechtler, K., Ponting, C.P., Allis, C.D., et al. (2000). Regulation of chromatin structure by site-specific histone H3 methyltransferases. Nature 406, 593-599.

Reddy, M.A., Zhang, E., and Natarajan, R. (2015). Epigenetic mechanisms in diabetic complications and metabolic memory. Diabetologia 58, 443-455.

Refinetti, R. (1996). The Body Temperature Rhythm of the Thirteen-Lined Ground 
Squirrel, Spermophilus tridecemlineatus. Physiol Zool 69, 270-275.

Rosenfeld, J.A., Wang, Z., Schones, D.E., Zhao, K., DeSalle, R., and Zhang, M.Q. (2009). Determination of enriched histone modifications in non-genic portions of the human genome. BMC Genomics 10, 143.

Rouble, A.N., and Storey, K.B. (2015). Characterization of the SIRT family of NAD+dependent protein deacetylases in the context of a mammalian model of hibernation, the thirteen-lined ground squirrel. Cryobiology 71, 334-343.

Rouble, A.N., Hefler, J., Mamady, H., Storey, K.B., and Tessier, S.N. (2013). Antiapoptotic signaling as a cytoprotective mechanism in mammalian hibernation. PeerJ 1, e29.

Rourke, B.C., Yokoyama, Y., Milsom, W.K., and Caiozzo, V.J. (2004). Myosin isoform expression and MAFbx mRNA levels in hibernating golden-mantled ground squirrels (Spermophilus lateralis). Physiol Biochem Zool 77, 582-593.

Rubin, C.S., and Rosen, O.M. (1975). Protein phosphorylation. Annu. Rev. Biochem. 44, $831-887$.

Rui, L. (2014). Energy metabolism in the liver. Compr Physiol 4, 177-197.

Russell, R.L., O'Neill, P.H., Epperson, L.E., and Martin, S.L. (2010). Extensive use of torpor in 13-lined ground squirrels in the fall prior to cold exposure. J Comp Physiol B, Biochem Syst Environ Physiol 180, 1165-1172.

Ruthenburg, A.J., Allis, C.D., and Wysocka, J. (2007). Methylation of lysine 4 on histone H3: intricacy of writing and reading a single epigenetic mark. Mol Cell 25, 15-30.

Saccani, S., Marazzi, I., Beg, A.A., and Natoli, G. (2004). Degradation of promoterbound p65/RelA is essential for the prompt termination of the nuclear factor kappaB response. J Exp Med 200, 107-113.

Samec, S., Seydoux, J., and Dulloo, A.G. (1998). Role of UCP homologues in skeletal muscles and brown adipose tissue: mediators of thermogenesis or regulators of lipids as fuel substrate? Faseb J 12, 715-724.

Sangiao-Alvarellos, S., Vázquez, M.J., Varela, L., Nogueiras, R., Saha, A.K., Cordido, F., López, M., and Diéguez, C. (2009). Central ghrelin regulates peripheral lipid metabolism in a growth hormone-independent fashion. Endocrinology 150, 45624574 .

Santos-Rosa, H., Schneider, R., Bannister, A.J., Sherriff, J., Bernstein, B.E., Emre, N.C.T., Schreiber, S.L., Mellor, J., and Kouzarides, T. (2002). Active genes are trimethylated at K4 of histone H3. Nature 419, 407-411.

Sarvan, S., Avdic, V., Tremblay, V., Chaturvedi, C.-P., Zhang, P., Lanouette, S., Blais, 
A., Brunzelle, J.S., Brand, M., and Couture, J.-F. (2011). Crystal structure of the trithorax group protein ASH2L reveals a forkhead-like DNA binding domain. Nat Struct Mol Biol 18, 857-859.

Satoh, T. (2014). Molecular mechanisms for the regulation of insulin-stimulated glucose uptake by small guanosine triphosphatases in skeletal muscle and adipocytes. International Journal of Molecular Sciences 15, 18677-18692.

Schotta, G., Sengupta, R., Kubicek, S., Malin, S., Kauer, M., Callén, E., Celeste, A., Pagani, M., Opravil, S., La Rosa-Velazquez, De, I.A., et al. (2008). A chromatinwide transition to H4K20 monomethylation impairs genome integrity and programmed DNA rearrangements in the mouse. Genes Dev 22, 2048-2061.

Schubert, H.L., Blumenthal, R.M., and Cheng, X. (2003). Many paths to methyltransfer: a chronicle of convergence. Trends Biochem Sci 28, 329-335.

Schwartz, C., Hampton, M., and Andrews, M.T. (2015). Hypothalamic gene expression underlying pre-hibernation satiety. Genes Brain Behav. 14, 310-318.

Schwartz, S., Meshorer, E., and Ast, G. (2009). Chromatin organization marks exonintron structure. Nat Struct Mol Biol 16, 990-995.

Scoumanne, A., and Chen, X. (2008). Protein methylation: a new mechanism of p53 tumor suppressor regulation. Histol. Histopathol. 23, 1143-1149.

Serkova, N.J., Rose, J.C., Epperson, L.E., Carey, H.V., and Martin, S.L. (2007). Quantitative analysis of liver metabolites in three stages of the circannual hibernation cycle in 13-lined ground squirrels by NMR. Physiol Genomics 31, 1524.

Shankar, S.R., Bahirvani, A.G., Rao, V.K., Bharathy, N., Ow, J.R., and Taneja, R. (2013). G9a, a multipotent regulator of gene expression. Epigenetics 8, 16-22.

Shavlakadze, T., and Grounds, M. (2006). Of bears, frogs, meat, mice and men: complexity of factors affecting skeletal muscle mass and fat. Bioessays 28, 9941009.

Shi, L., Sun, L., Li, Q., Liang, J., Yu, W., Yi, X., Yang, X., Li, Y., Han, X., Zhang, Y., et al. (2011). Histone demethylase JMJD2B coordinates H3K4/H3K9 methylation and promotes hormonally responsive breast carcinogenesis. Proc Natl Acad Sci U S A $108,7541-7546$.

Shinsky, S.A., Hu, M., Vought, V.E., Ng, S.B., Bamshad, M.J., Shendure, J., and Cosgrove, M.S. (2014). A non-active-site SET domain surface crucial for the interaction of MLL1 and the RbBP5/Ash2L heterodimer within MLL family core complexes. J. Mol. Biol. 426, 2283-2299.

Sidler, C., Woycicki, R., Li, D., Wang, B., Kovalchuk, I., and Kovalchuk, O. (2014). A 
role for SUV39H1-mediated H3K9 trimethylation in the control of genome stability and senescence in WI38 human diploid lung fibroblasts. Aging (Albany NY) 6 , 545-563.

Sims, R.J., Chen, C.-F., Santos-Rosa, H., Kouzarides, T., Patel, S.S., and Reinberg, D. (2005). Human but not yeast CHD1 binds directly and selectively to histone H3 methylated at lysine 4 via its tandem chromodomains. J Biol Chem 280, 4178941792.

Snowden, A.W., Gregory, P.D., Case, C.C., and Pabo, C.O. (2002). Gene-specific targeting of $\mathrm{H} 3 \mathrm{~K} 9$ methylation is sufficient for initiating repression in vivo. Curr Biol 12, 2159-2166.

Son, M.J., Kim, W.K., Park, A., Oh, K.-J., Kim, J.-H., Han, B.S., Kim, I.C., Chi, S.-W., Park, S.G., Lee, S.C., et al. (2016). Set7/9, a methyltransferase, regulates the thermogenic program during brown adipocyte differentiation through the modulation of p53 acetylation. Mol Cell Endocrinol 431, 46-53.

Sookoian, S., and Pirola, C.J. (2013). Epigenetics of Insulin Resistance: An Emerging Field in Translational Medicine. Curr. Diab. Rep. 13, 229-237.

Srere, H.K., Wang, L.C.H., and Martin, S.L. (1992). Central Role for Differential GeneExpression in Mammalian Hibernation. Proc Natl Acad Sci U S A 89, 7119-7123.

Staples, J.F. (2014). Metabolic suppression in mammalian hibernation: the role of mitochondria. J Exp Biol 217, 2032-2036.

Stark, G.R., Wang, Y., and Lu, T. (2010). Lysine methylation of promoter-bound transcription factors and relevance to cancer. Cell Res 21,375-380.

Storey, K.B. (1987). Regulation of Liver-Metabolism by Enzyme Phosphorylation During Mammalian Hibernation. J Biol Chem 262, 1670-1673.

Storey, K.B. (1990). Life in a frozen state: adaptive strategies for natural freeze tolerance in amphibians and reptiles. Am. J. Physiol. 258, R559-R568.

Storey, K.B. (1997). Metabolic regulation in mammalian hibernation: enzyme and protein adaptations. Comp Biochem Physiol a Physiol 118, 1115-1124.

Storey, K.B. (2003). Mammalian hibernation - Transcriptional and translational controls. Adv. Exp. Med. Biol. 543, 21-38.

Storey, K.B. (2010). Out Cold: Biochemical Regulation of Mammalian Hibernation - A Mini-Review. Gerontology 56, 220-230.

Storey, K.B. (2015). Regulation of hypometabolism: insights into epigenetic controls. J Exp Biol 218, 150-159. 
Storey, K.B., and Storey, J.M. (2004a). Mammalian Hibernation: Biochemical Adaptation And Gene Expression. In Functional Metabolism: Regulation and Adaptation, K.B. Storey, ed. (Hobocken, NJ: John Wiley \& Sons, Inc.), pp. 1-30.

Storey, K.B., and Storey, J.M. (2004b). Metabolic rate depression in animals: transcriptional and translational controls. Biol Rev Camb Philos Soc 79, 207-233.

Storey, K.B., and Storey, J.M. (2007). Tribute to PL Lutz: putting life onpause'molecular regulation of hypometabolism. J Exp Biol 210, 1700-1714.

Storey, K.B., and Storey, J.M. (2010). Metabolic Rate Depression: the Biochemistry of Mammalian Hibernation. In Advances in Clinical Chemistry, G.S. Makowski, ed. (Burlington, VT: Academic Press), pp. 77-108.

Storey, K.B., and Storey, J.M. (2011). Heat shock proteins and hypometabolism: adaptive strategy for proteome preservation. Research and Reports in Biology 2, 57-68.

Storey, K.B., and Storey, J.M. (2013). Molecular biology of freezing tolerance. Compr Physiol 3, 1283-1308.

Storey, K.B., Heldmaier, G., and Rider, M.H. (2010). Mammalian Hibernation: Physiology, Cell Signaling, and Gene Controls on Metabolic Rate Depression. In Dormancy and Resistance in Harsh Environments, E. Lubzens, J. Cerda, and M. Clark, eds. (Berlin, Heidelberg: Springer Berlin Heidelberg), pp. 227-252.

Stram, A.R., and Payne, R.M. (2016). Post-translational modifications in mitochondria: protein signaling in the powerhouse. Cell. Mol. Life Sci. [Epubaheadofprint].

Subramanian, K., Jia, D., Kapoor-Vazirani, P., Powell, D.R., Collins, R.E., Sharma, D., Peng, J., Cheng, X., and Vertino, P.M. (2008). Regulation of estrogen receptor alpha by the SET7 lysine methyltransferase. Mol Cell 30, 336-347.

Tachibana, M., Sugimoto, K., Fukushima, T., and Shinkai, Y. (2001). Set domaincontaining protein, G9a, is a novel lysine-preferring mammalian histone methyltransferase with hyperactivity and specific selectivity to lysines 9 and 27 of histone H3. J Biol Chem 276, 25309-25317.

Takaya, T., Kawamura, T., Morimoto, T., Ono, K., Kita, T., Shimatsu, A., and Hasegawa, K. (2008). Identification of p300-targeted acetylated residues in GATA4 during hypertrophic responses in cardiac myocytes. J Biol Chem 283, 9828-9835.

Tang, Z., Chen, W.-Y., Shimada, M., Nguyen, U.T.T., Kim, J., Sun, X.-J., Sengoku, T., McGinty, R.K., Fernandez, J.P., Muir, T.W., et al. (2013). SET1 and p300 act synergistically, through coupled histone modifications, in transcriptional activation by p53. Cell 154, 297-310.

Taylor-Burt, K.R., Monroy, J., Pace, C., Lindstedt, S., and Nishikawa, K.C. (2015). Shiver me titin! Elucidating titin's role in shivering thermogenesis. J Exp Biol 218, 
694-702.

Tessier, S.N., and Storey, K.B. (2010). Expression of myocyte enhancer factor-2 and downstream genes in ground squirrel skeletal muscle during hibernation. Mol Cell Biochem 344, 151-162.

Tessier, S.N., and Storey, K.B. (2012). Myocyte enhancer factor-2 and cardiac muscle gene expression during hibernation in thirteen-lined ground squirrels. Gene 501, 8 16.

Tessier, S.N., and Storey, K.B. (2014). To be or not to be: the regulation of mRNA fate as a survival strategy during mammalian hibernation. Cell Stress Chaperones 19, 763-776.

Tessier, S.N., and Storey, K.B. (2016). Lessons from mammalian hibernators: molecular insights into striated muscle plasticity and remodeling. Biomol Concepts 7, 69-92.

Tian, W., and Xu, Y. (2015). Decoding liver injury: A regulatory role for histone modifications. Int. J. Biochem. Cell Biol. 67, 188-193.

Toshinai, K., Mondal, M.S., Nakazato, M., Date, Y., Murakami, N., Kojima, M., Kangawa, K., and Matsukura, S. (2001). Upregulation of Ghrelin expression in the stomach upon fasting, insulin-induced hypoglycemia, and leptin administration. Biochem Biophys Res Commun 281, 1220-1225.

Trivedi, C.M., Zhu, W., Wang, Q., Jia, C., Kee, H.J., Li, L., Hannenhalli, S., and Epstein, J.A. (2010). Hopx and Hdac2 interact to modulate Gata4 acetylation and embryonic cardiac myocyte proliferation. Dev. Cell 19, 450-459.

Tu, P., Li, X., Ma, B., Duan, H., Zhang, Y., Wu, R., Ni, Z., Jiang, P., Wang, H., Li, M., et al. (2015). Liver histone $\mathrm{H} 3$ methylation and acetylation may associate with type 2 diabetes development. J. Physiol. Biochem. 71, 89-98.

Ullius, A., Lüscher-Firzlaff, J., Costa, I.G., Walsemann, G., Forst, A.H., Gusmao, E.G., Kapelle, K., Kleine, H., Kremmer, E., Vervoorts, J., et al. (2014). The interaction of MYC with the trithorax protein ASH2L promotes gene transcription by regulating H3K27 modification. Nucleic Acids Res 42, 6901-6920.

van Breukelen, F., and Martin, S.L. (2002). Reversible depression of transcription during hibernation. J Comp Physiol B 172, 355-361.

van Breukelen, F. (2016). Applying systems-level approaches to elucidate regulatory function during mammalian hibernation. Temperature 3,1-3.

van Breukelen, F., and Martin, S.L. (2001). Translational initiation is uncoupled from elongation at $18^{\circ} \mathrm{C}$ during mammalian hibernation. Am J Physiol Regul Integr Comp Physiol 281, R1374-R1379. 
Vermillion, K.L., Jagtap, P., Johnson, J.E., Griffin, T.J., and Andrews, M.T. (2015). Characterizing Cardiac Molecular Mechanisms of Mammalian Hibernation via Quantitative Proteogenomics. J. Proteome Res. 14, 4792-4804.

Villén, J., Beausoleil, S.A., Gerber, S.A., and Gygi, S.P. (2007). Large-scale phosphorylation analysis of mouse liver. Proc Natl Acad Sci U S A 104, 14881493.

Voelkel, T., Andresen, C., Unger, A., Just, S., Rottbauer, W., and Linke, W.A. (2013). Lysine methyltransferase Smyd2 regulates Hsp90-mediated protection of the sarcomeric titin springs and cardiac function. BBA-Gene Regul Mech 1833, 812822.

Vucetic, M., Stancic, A., Otasevic, V., Jankovic, A., Korac, A., Markelic, M., Velickovic, K., Golic, I., Buzadzic, B., Storey, K.B., et al. (2013). The impact of cold acclimation and hibernation on antioxidant defenses in the ground squirrel (Spermophilus citellus): an update. Free Radic Biol Med 65, 916-924.

Wadhwa, R., Taira, K., and Kaul, S.C. (2002). An Hsp70 family chaperone, mortalin/mthsp70/PBP74/Grp75: what, when, and where? Cell Stress Chaperones 7, 309-316.

Wagner, E.J., and Carpenter, P.B. (2012). Understanding the language of Lys36 methylation at histone H3. Nat Rev Mol Cell Biol 13, 115-126.

Wang, D., Zhou, J., Liu, X., Lu, D., Shen, C., Du, Y., Wei, F.-Z., Song, B., Lu, X., Yu, Y., et al. (2013). Methylation of SUV39H1 by SET7/9 results in heterochromatin relaxation and genome instability. Proc Natl Acad Sci U.S.a. 110, 5516-5521.

Wang, L.C.H. (1979). Time patterns and metabolic rates of natural torpor in the Richardson's ground squirrel. Can J Zool 57, 149-155.

Wang, P., Walter, R.D., Bhat, B.G., and Florant, G.L. (1997). Seasonal changes in enzymes of lipogenesis and triacylglycerol synthesis in the golden-mantled ground squirrel (Spermophilus lateralis). J Comp Physiol B 118B, 261-267.

Wang, Y.-C., Peterson, S.E., and Loring, J.F. (2014). Protein post-translational modifications and regulation of pluripotency in human stem cells. Cell Res 24, 143-160.

Wang, Z., Zang, C., Rosenfeld, J.A., Schones, D.E., Barski, A., Cuddapah, S., Cui, K., Roh, T.-Y., Peng, W., Zhang, M.Q., et al. (2008). Combinatorial patterns of histone acetylations and methylations in the human genome. Nat Genet 40, 897-903.

West, L.E., and Gozani, O. (2011). Regulation of p53 function by lysine methylation. Epigenomics 3, 361-369.

Whitten, B.K., and Klain, G.J. (1968). Protein metabolism in hepatic tissue of hibernating 
and arousing ground squirrels. Am J Physiol 214, 1360-1362.

Williams, C.T., Goropashnaya, A.V., Buck, C.L., Fedorov, V.B., Kohl, F., Lee, T.N., and Barnes, B.M. (2011). Hibernating above the permafrost: effects of ambient temperature and season on expression of metabolic genes in liver and brown adipose tissue of arctic ground squirrels. J Exp Biol 214, 1300-1306.

Wilson, B.E., Deeb, S., and Florant, G.L. (1992). Seasonal changes in hormone-sensitive and lipoprotein lipase mRNA concentrations in marmot white adipose tissue. Am J Physiol 262, R177-R181.

Wood, H. (2015). Neurodegenerative disease: RBM3, a protein upregulated during hibernation, provides new insights into neurodegeneration. Nat Rev Neurol 11, 124-124.

Woods, S.C., and Porte, D. (1978). The central nervous system, pancreatic hormones, feeding, and obesity. Adv Metab Disord 9, 283-312.

Wozniak, G.G., and Strahl, B.D. (2014). Hitting the "mark": interpreting lysine methylation in the context of active transcription. BBA-Gene Regul Mech 1839, $1353-1361$.

Wu, C.-W., and Storey, K.B. (2012a). Regulation of the mTOR signaling network in hibernating thirteen-lined ground squirrels. J Exp Biol 215, 1720-1727.

Wu, C.-W., and Storey, K.B. (2012b). Pattern of cellular quiescence over the hibernation cycle in liver of thirteen-lined ground squirrels. Cell Cycle 11, 1714-1726.

Wu, C.-W., and Storey, K.B. (2016). Life in the cold: links between mammalian hibernation and longevity. Biomol Concepts 7, 1-12.

Wu, C.-W., Biggar, K.K., and Storey, K.B. (2013). Biochemical adaptations of mammalian hibernation: exploring squirrels as a perspective model for naturally induced reversible insulin resistance. Braz J Med Biol Res 46, 1-13.

Wu, C.-W., Biggar, K.K., Zhang, J., Tessier, S.N., Pifferi, F., Perret, M., and Storey, K.B. (2015). Induction of Antioxidant and Heat Shock Protein Responses During Torpor in the Gray Mouse Lemur, Microcebus murinus. Genomics Proteomics Bioinformatics 13, 119-126.

Wu, H., Chen, X., Xiong, J., Li, Y., Li, H., Ding, X., Liu, S., Chen, S., Gao, S., and Zhu, B. (2011). Histone methyltransferase G9a contributes to H3K27 methylation in vivo. Cell Res 21,365-367.

Xu, Y., Shao, C., Fedorov, V.B., Goropashnaya, A.V., Barnes, B.M., and Yan, J. (2013). Molecular signatures of mammalian hibernation: comparisons with alternative phenotypes. BMC Genomics 14. 
Yamane, K., Toumazou, C., Tsukada, Y.-I., Erdjument-Bromage, H., Tempst, P., Wong, J., and Zhang, Y. (2006). JHDM2A, a JmjC-Containing H3K9 Demethylase, Facilitates Transcription Activation by Androgen Receptor. Cell 125, 483-495.

Yang, C.-X., He, Y., Gao, Y.-F., Wang, H.-P., and Goswami, N. (2014). Changes in calpains and calpastatin in the soleus muscle of Daurian ground squirrels during hibernation. Comp Biochem Physiol, Part a Mol Integr Physiol 176, 26-31.

Yang, X.-D., Huang, B., Li, M., Lamb, A., Kelleher, N.L., and Chen, L.-F. (2009). Negative regulation of NF-kappaB action by Set9-mediated lysine methylation of the RelA subunit. Embo J 28, 1055-1066.

Yang, X.-D., Tajkhorshid, E., and Chen, L.-F. (2010). Functional interplay between acetylation and methylation of the RelA subunit of NF-kappaB. Mol Cell Biol 30, 2170-2180.

Yang, Z., Augustin, J., Hu, J., and Jiang, H. (2015). Physical Interactions and Functional Coordination between the Core Subunits of Set1/Mll Complexes and the Reprogramming Factors. PLoS ONE 10, e0145336.

Yeh, I., Tam, C.F., Catuira, E., Le, T.T., Papa, V., Pena, L., Vasquez, M., Vu, C., Wang, S., and Lopez, G.A. (1995). Changes in various plasma lipid components, glucose, and insulin in Spermophilus lateralis during hibernation. Comp Biochem Physiol B, Biochem Mol Biol 111, 651-663.

Yokoyama, A., Fujiki, R., Ohtake, F., and Kato, S. (2011). Regulated histone methyltransferase and demethylase complexes in the control of genes by nuclear receptors. Cold Spring Harb Symp Quant Biol 76, 165-173.

Yokoyama, A., Wang, Z., Wysocka, J., Sanyal, M., Aufiero, D.J., Kitabayashi, I., Herr, W., and Cleary, M.L. (2004). Leukemia proto-oncoprotein MLL forms a SET1-like histone methyltransferase complex with menin to regulate Hox gene expression. Mol Cell Biol 24, 5639-5649.

Zhang, W.-J., Wu, X.-N., Shi, T.-T., Xu, H.-T., Yi, J., Shen, H.-F., Huang, M.-F., Shu, X.-Y., Wang, F.-F., Peng, B.-L., et al. (2016). Regulation of Transcription Factor Yin Yang 1 by SET7/9-mediated Lysine Methylation. Sci Rep 6, 21718.

Zhang, X., Huang, Y., and Shi, X. (2015). Emerging roles of lysine methylation on nonhistone proteins. Cell Mol Life Sci 72, 4257-4272.

Zhang, X., Wen, H., and Shi, X. (2012a). Lysine methylation: beyond histones. Acta Biochim Biophys Sin 44, 14-27.

Zhang, X., Yang, Z., Khan, S.I., Horton, J.R., Tamaru, H., Selker, E.U., and Cheng, X. (2003). Structural basis for the product specificity of histone lysine methyltransferases. Mol Cell 12, 177-185. 
Zhang, X., Zhou, D., Strakovsky, R., Zhang, Y., and Pan, Y.-X. (2012b). Hepatic cellular senescence pathway genes are induced through histone modifications in a dietinduced obese rat model. Am J Physiol Gastrointest Liver Physiol 302, G558G564.

Zhang, Y., and Reinberg, D. (2001). Transcription regulation by histone methylation: interplay between different covalent modifications of the core histone tails. Genes Dev 15, 2343-2360.

Zhang, Z., and Zhang, M.Q. (2011). Histone modification profiles are predictive for tissue/cell-type specific expression of both protein-coding and microRNA genes. BMC Bioinformatics 12, 155.

Zhao, S., Xu, W., Jiang, W., Yu, W., Lin, Y., Zhang, T., Yao, J., Zhou, L., Zeng, Y., Li, H., et al. (2010). Regulation of Cellular Metabolism by Protein Lysine Acetylation. Science 327, 1000-1004.

Zhou, Q., Shaw, P.G., and Davidson, N.E. (2009). Epigenetics meets estrogen receptor: regulation of estrogen receptor by direct lysine methylation. Endocr Relat Cancer 16, 319-323.

Zhu, X., Smith, M.A., Perry, G., Wang, Y., Ross, A.P., Zhao, H.W., LaManna, J.C., and Drew, K.L. (2005). MAPKs are differentially modulated in arctic ground squirrels during hibernation. J Neurosci Res 80, 862-868. 


\section{Appendices}




\section{Appendix A: Comparisons of Orthologous Protein Sequences from Ictidomys tridecemlineatus and Homo sapien}

The following pages compare the sequences of the proteins investigated in this study to determine amino acid sequence identity. Protein sequences were found using the National Center for Biotechnology Information protein database and comparisons were made using Clustal Omega software courtesy of The European Bioinformatics Institute.

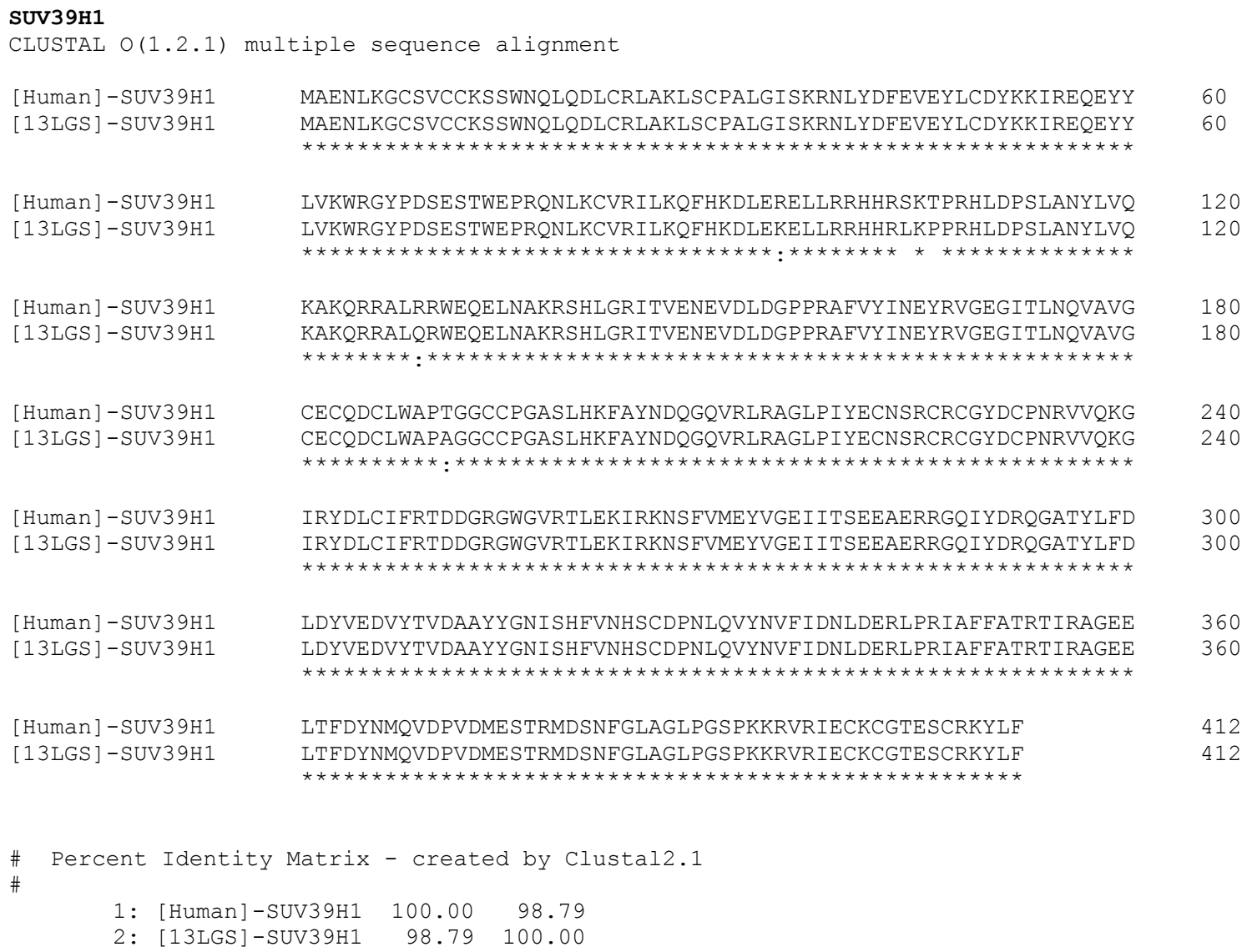


SMYD2

CLUSTAL O(1.2.1) multiple sequence alignment:

[Human]-SMYD2

[13LGS ]-SMYD2

MLVFEKHALETQRLFFTGTQGAIKVRERASVCRRGGQAARSCALLARWLRLRAGSNQLIA

[Human]-SMYD2

[13LGS]-SMYD2

YEEWAIGDGAEQEERAGSARHSAREAKPLAARERSERAPPGSGTGTLSPRGSGSGPPKVI

[Human]-SMYD2

[13LGS]-SMYD2

----------------MRAEGLGGLERFCSPGKGRGLRALQPFQVGDLLFSCPAYAY NKDLRTVWRAVWAPFQLPGARHPGHGGLERFCSPGKGRGLRALQPFQVGDLLFSCPAYAY

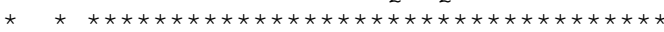

180

[Human]-SMYD2

VLTVNERGNHCEYCFTRKEGLSKCGRCKQAFYCNVECQKEDWPMHKLECS PMVVFGENWN VLTVNERGNHCEYCFARKEGLSKCGRCKQAFYCNVECQKEDWPMHKLECSPMVVFGENWS

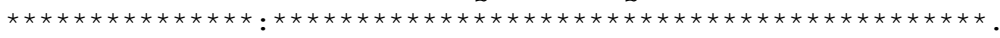

[Human]-SMYD2

PSETVRLTARILAKQKIHPERTPSEKLLAVKEFESHLDKLDNEKKDLIQSDIAALHHFYS PSETVRLTARILAKQKI HPERTPSEKLLAVKEFESHLDKLDNEKKDLIQSDIAALHQFYS

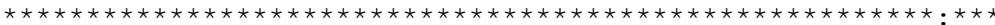

[Human]-SMYD2

[13LGS ]-SMYD2

KHLGFPDNDSLVVLFAQVNCNGFTIEDEELSHLGSAIFPDVALMNHSCCPNVIVTYKGTL KHLEFPDHSSLVVLFAQVNCNGFTIEDEELSHLGSAIFPDVALMNHSCCPNVIVTYKGTL

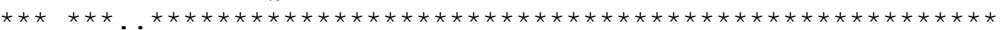

[Human]-SMYD2

[13LGS]-SMYD2

AEVRAVQEIKPGEEVFTSYIDLLYPTEDRNDRLRDSYFFTCECQECTTKDKDKAKVEIRK AEVRAVQEISPGEEVFTSYIDLLYPTEDRNDRLRDSYFFTCECQECTTKDKDTAKVEIRK

[Human]-SMYD2

LSDPPKAEAIRDMVRYARNVIEEFRRAKHYKSPSELLEICELSQEKMSSVFEDSNVYMLH LSDPPKAEAIRDMVRYARNVIEEFRRAKHYKSPSELLEICELSQEKMSSVFEDSNVYMLH

[Human]-SMYD2

MMYQAMGVCLYMQDWEGALQYGQKI IKPYSKHYPLYSLNVASMWLKLGRLYMGLEHKAAG

\# Percent Identity Matrix - created by Clustal2.1

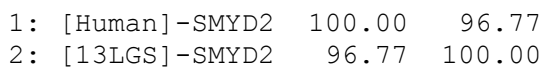


SET 8

CLUSTAL O(1.2.1) multiple sequence alignment

[Human]-SET 8

[13LGS]-SET 8

MNEQNCWRGQGVGDAKLGELDCPGHKVRCRGQRSGSGQHQEEPEVPDQVHSARTSSCKTK

0

MVOTHRGGVGAKLGELDCPGKVRCRGQRSGSGQHQEEPEVPDQVHSARISSCKIKK

60

[Human]-SET 8

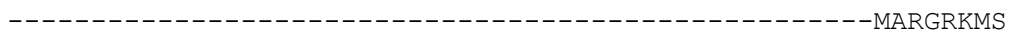
LTEGELQHREREGLAVLQWQLDCPS ISANQRQETVKFKPPPISSAGTCPESVCRKSGRWP

KPRAVEAAAAAAAVAATAPGPEMVERRGPGRPRTDGENVFTGQSKIYSYMS PNKCSGMRF RPRRGGGEKGGLSQPLRLSRPAAFGETGGGA--NQGEQLQLTGA--VGYSCCNFFRKLGF PGDPGGDRAGPWLEKRVPRHLPEQPSAQKPARTPALRGWRGWACAAS---AGDVPASKKR

$$
\text { * : }: \text { * } \quad \text { * . . . . . ** }
$$

[Human]-SET 8

NAGNAVRSAMKSEEQKIKDARKGPLVPFPNQKSEAAEPPKTPPSSCDSTNAAIAKQALKK SAGNAIRSSLKSEEQKIKDARRGPLAPFPNQKSEAAEPPKTPTSTCDSTNAAIAKQALKK

PIKGKQAPRKKAQGKTQQNRKLTDFYPVRRSSRKSKAELQSEERKRIDELIESGKEEGMK PLKGKQTPRKKAQGKTQQNRKLTDFYPVRRSSRKSKAELQSEERKRIDELIESGKEEGMK

\# Percent Identity Matrix - created by Clustal2.1

\#
1: [Human]-SET8
100.00
74.20
2: [13LGS]-SET8 $74.20 \quad 100.00$ 


\section{ASH2L}

CLUSTAL O(1.2.1) multiple sequence alignment

[Human]-ASH2L

[13LGS]-ASH2L

[Human]-ASH2L

[13LGS]-ASH2L

[Human]-ASH2L

[13LGS]-ASH2I

[Human]-ASH2L

[13LGS]-ASH2L

[Human]-ASH2L

[13LGS]-ASH2L

[Human]-ASH2L

[13LGS]-ASH2I

[Human]-ASH2I

[13LGS]-ASH2I

[Human]-ASH2L

[13LGS]-ASH2I

[Human ]-ASH2I

[13LGS ]-ASH2L

[Human]-ASH2L [13LGS]-ASH2L

[Human]-ASH2L [13LGS]-ASH2I
MAAAGAGPGOEAGAGPGPGAVANATGAEEGEMKPVAAGAAAPPGEGISAAPTVEPSSGEA MAAAGTGPGPGAGSGPGPVAAANAITAEEGETKPVTAVAATPAGEGTSAAPATEPSSGEA

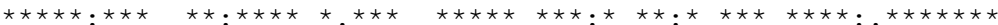

EGGEANLVDVSGGLETESSNGKDTLEGAGDTSEVMDTQAGSVDEENGRQLGEVELQCGIC ESGDANLVDVSGGLETESSNGKDTLEGTGDTSEVMDTQAGSVDEENGRQLGEVELQCGIC

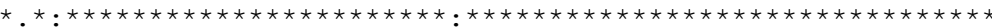

TKWFTADTFGIDTSSCLPFMTNYSFHCNVCHHSGNTYFLRKQANLKEMCLSALANLTWQS TKWFTADTFGIDTSSCLPFMTNYSFHCNVCHHSGNTYFLRKOANLKEMCLSALANLTWOS

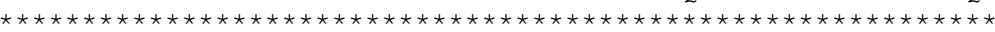

RTQDEHPKTMFSKDKDI I PFIDKYWECMTTRQRPGKMTWPNNIVKTMSKERDVFLVKEHP RTODEHPKTMFSKDKDI I PFIDKYWECMTTRORPGKMTWPNNIVKTMSKERDVFLVKEHP

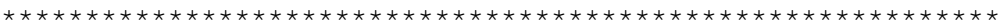

DPGSKDPEEDYPKFGLLDQDLSNIGPAYDNQKQSSAVSTSGNLNGGIAAGSSGKGRGAKR DPGSKDPEEDYPKFGLLDQDLSNIGPAYDNQKQSSTVSTSGNLNGGIAAGSSGKGRGAKR

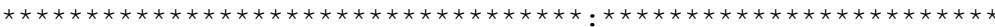

KQQDGGTTGTTKKARSDPLFSAQRLPPHGYPLEHPFNKDGYRYILAEPDPHAPDPEKLEL

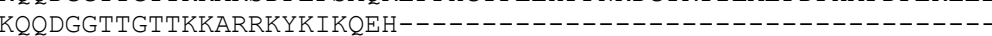

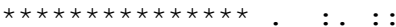

DCWAGKPIPGDLYRACLYERVLLALHDRAPOLKI SDDRLTVVGEKGYSMVRASHGVRKGA -LVMLETPQLKISDDRLTVIGEKGYSMVRASHGVRKGA

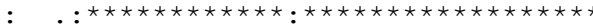

WYFEITVDEMP PDTAARLGWSQPLGNLQAPLGYDKFSYSWRSKKGTKFHQSIGKHYSSGY WYFEITVDEMPPDTAARLGWSQPLGNLQAPLGYDKFSYSWRSKKGTKFHQS IGKHYSSGY

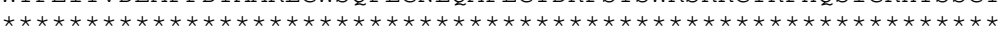

GQGDVLGFY INLPEDTETAKSLPDTYKDKALIKFKSYLYFEEKDFVDKAEKSLKQTPHSE GQGDVLGFYINLPEDTETAKSLPDTYKDKALIKFKSYLYFEEKDFVDKAEKSLKQTPHSE

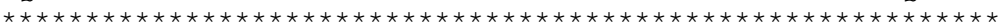

I IFYKNGVNQGVAYKDIFEGVYFPAISLYKSCTVS INFGPCFKYPPKDLTYRPMSDMGWG I I FYKNGVNQGVAYKDIFEGVYFPAISLYKSCTVS INFGPCFKYPPKDLTYHPMSDMGWG

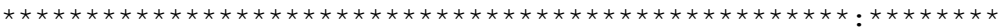

60

60

120
AVVEHTLADVLYHVETEVDGRRSPPWEP 628 AVVEHTLADVLYHVETEVDGRRSPPWEP 569

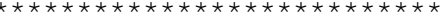

\# Percent Identity Matrix - created by Clustal2.1

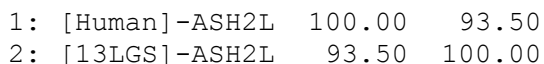


RBBP5, isoform $\mathrm{x} 1$

CLUSTAL O(1.2.1) multiple sequence alignment

[Human]-RBBP5

[13LGS ]-RBBP5

[Human] -RBBP5

[13LGS ]-RBBP5

[Human]-RBBP5

[13LGS]-RBBP5

[Human]-RBBP5

[13LGS]-RBBP5

[Human]-RBBP5

[13LGS ]-RBBP5

[Human]-RBBP5

[13LGS]-RBBP 5

[Human]-RBBP5

[13LGS ]-RBBP5

[Human]-RBBP5

[13LGS]-RBBP5

[Human]-RBBP5

[13LGS]-RBBP5
MNLELLESFGQNYPEEADGTLDCISMALTCTFNRWGTLLAVGCNDGRIVIWDFLTRGIAK MNLELLESFGQNYPEEADGTLDCISMALTCTFNRWGTLLAVGCNDGRIVIWDFLTRGIAK

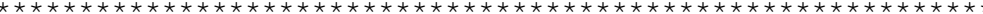

I ISAHIHPVCSLCWSRDGHKLVSASTDNIVSQWDVLSGDCDQRFRFPSPILKVQYHPRDQ I ISAH IHPVCSLCWSRDGHKLVSASTDNIVSQWDVLSGDCDQRFRFPSPILKVQYHPRDQ

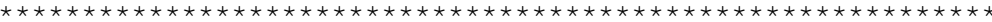

NKVLVCPMKSAPVMLTLSDSKHVVLPVDDDSDLNVVASFDRRGEYIYTGNAKGKILVLKT NKVLVCPMKSAPVMLTLSDSKHVVLPVDDDSDLNVVASFDRRGEYIYTGNAKGKILVLKT

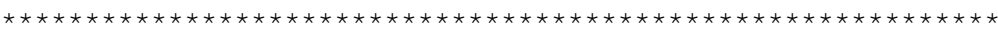
DSQDLVASFRVTTGTSNTTAIKS IEFARKGSCFLINTADRI IRVYDGREILTCGRDGEPE DSQDLVASFRVTTGTSNTTAIKS IEFARKGSCFLINTADRI IRVYDGREI LTCGRDGEPE

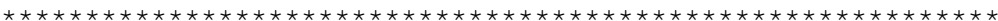

PMQKLQDLVNRTPWKKCCFSGDGEY IVAGSARQHALY IWEKS I GNLVKILHGTRGELLLD PMQKLQDLVNRTPWKKCCFSGDGEY IVAGSARQHALY IWEKS I GNLVKILHGTRGELLLD

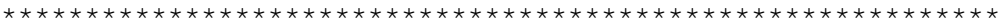

VAWHPVRPI IASISSGVVS IWAQNQVENWSAFAPDFKELDENVEYEERESEFDIEDEDKS VAWHPVRPI IASISSGVVS IWAQNQVENWSAFAPDFKELDENVEYEERESEFDIEDEDKS

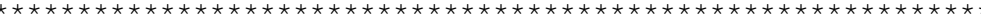

EPEQTGADAAEDEEVDVTSVDPIAAFCSSDEELEDSKALLYLPIAPEVEDPEENPYGPPP EPEQTGADAAEDEEVDVTSVDPIAAFCSSDEELEDSKALLYLPIAPEVEDPEENPYGPPP

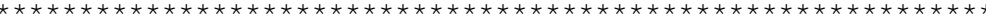

DAVQTSLMDEGASSEKKRQSSADGSQPPKKKPKTTNIELQGVPNDEVHPLLGVKGDGKSK DAVQTSLMDEGAGSEKKRQSSTDGSQPPKKKPKTTNIELQGVPNDEVHPLLGVKGDGKSK

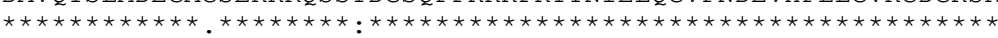

KKQAGRPKGSKGKEKDSPFKPKLYKGDRGLPLEGSAKGKVQAELSQPLTAGGAISELL KKQAGRPKGSKGKEKDSPFKPKLYKGDRGLPLEGSTKGKVQAELSQPLTAGGAISELL

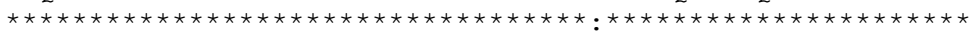

360 360

420

420

60

60

120

180

180

240

\# Percent Identity Matrix - created by Clustal2.1
1: [Human]-RBBP5 $100.00 \quad 99.44$
2: [13LGS]-RBBP5 $99.44 \quad 100.00$ 
RBBP5, isoform $\mathrm{X} 2$

CLUSTAL O(1.2.1) multiple sequence alignment

[Human]-RBBP5

[13LGS ]-RBBP5

[Human]-RBBP5

[13LGS ]-RBBP5

[Human]-RBBP5

[13LGS]-RBBP5

[Human]-RBBP5

[13LGS]-RBBP5

[Human]-RBBP5

[13LGS ]-RBBP5

[Human]-RBBP5

[13LGS]-RBBP 5

[Human]-RBBP5

[13LGS ]-RBBP5

[Human]-RBBP5

[13LGS]-RBBP5

[Human]-RBBP 5

[13LGS ]-RBBP5
MNLELLESFGQNYPEEADGTLDCISMALTCTFNRWGTLLAVGCNDGRIVIWDFLTRGIAK MNLELLESFGQNYPEEADGTLDCISMALTCTFNRWGTLLAVGCNDGRIVIWDFLTRGIAK

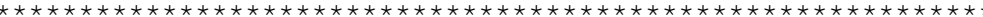

I ISAHIHPVCSLCWSRDGHKLVSASTDNIVSQWDVLSGDCDQRFRFPSPILKVQYHPRDQ I ISAH IHPVCSLCWSRDGHKLVSASTDNIVSQWDVLSGDCDQRFRFPSPILKVQYHPRDQ

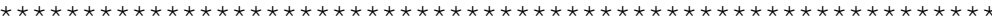

NKVLVCPMKSAPVMLTLSDSKHVVLPVDDDSDLNVVASFDRRGEYIYTGNAKGKILVLKT NKVLVCPMKSAPVMLTLSDSKHVVLPVDDDSDLNVVASFDRRGEYIYTGNAKGKILVLKT

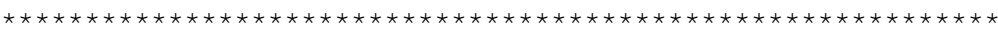
DSQDLVASFRVTTGTSNTTAIKS IEFARKGSCFLINTADRI IRVYDGREILTCGRDGEPE DSQDLVASFRVTTGTSNTTAIKS IEFARKGSCFLINTADRI IRVYDGREI LTCGRDGEPE

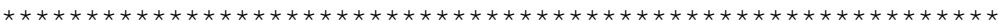

PMQKLQDLVNRTPWKKCCFSGDGEY IVAGSARQHALY IWEKS I GNLVKI LHGTRGELLLD PMQKLQDLVNRTPWKKCCFSGDGEY IVAGSARQHALY IWEKS I GNLVKILHGTRGELLLD

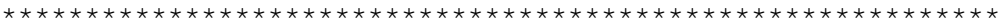

VAWHPVRPI IASISSGVVS IWAQNQVENWSAFAPDFKELDENVEYEERESEFDIEDEDKS VAWHPVRPI IASISSGVVS IWAQNQVENWSAFAPDFKELDENVEYEERESEFDIEDEDKS

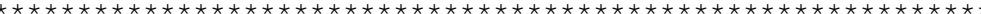

360 360

EPEQTGADAAEDEEVDVTSVDPIAAFCSSDEELEDSKALLYLPIAPEVEDPEENPYGPPP EPEQTGADAAEDEEVDVTSVDPIAAFCSSDEELEDSKALLYLPIAPEVEDPEENPYGPPP

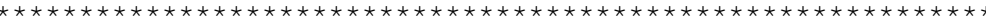

DAVQTSLMDEGASSEKKRQSSADGSQPPKKKPKTTNIELQGVPNDEVHPLLGVKGDGKSK DAVQTSLMDEGAGSEKKRQSSTDGSQPPKKKPKTTNIELQGVPNDEVHPLLGVKGDGKSK 480

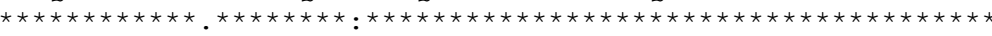

\#

KKQAGRPKGSKAGGAISELL $\quad 500$ KKQAGRPKGSKAGGAISELL 500

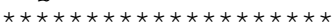

Percent Identity Matrix - created by Clustal2.1

1: [Human]-RBBP5 $100.00 \quad 99.60$

2: [13LGS]-RBBP5 $99.60 \quad 100.00$ 
G9a

CLUSTAL O(1.2.1) multiple sequence alignment

[Human]-G9a

[13LGS]-G9a

[Human]-G9a

[13LGS]-G9a

[Human]-G9a

[13LGS]-G9a

[Human]-G9a

[13LGS]-G9a

[Human]-G9a

[13LGS]-G9a

[Human]-G9a

[13LGS]-G9a

[Human]-G9a

[13LGS]-G9a

[Human]-G9a

[13LGS]-G9a

[Human]-G9a

[13LGS]-G9a

[Human]-G9a

[13LGS]-G9a

[Human]-G9a

[13LGS ]-G9a

[Human]-G9a

[13LGS]-G9a

[Human]-G9a

[13LGS]-G9a

[Human]-G9a

[13LGS]-G9a

[Human]-G9a

[13LGS]-G9a

[Human]-G9a

[13LGS]-G9a

[Human]-G9a

[13LGS]-G9a
MRGLPRGRGLMRARGRGRAAPPGSRGRGRGGPHRGRGRPRSLLSLPRAOASWTPOLSTGL -------GLMRARGRGRAAPPGSRGRGRGGPHRGRGRPRSLLSLPRAQASWAPQLPTGL

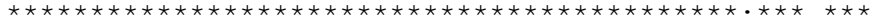

TSPPVPCLPSOGEAPAEMGALLLEKETRGATERVHGSLGDTPRSEETLPKATPDSLEPAG TSPPVPCLPSQGEAPAEMGALLLEKEPRGATERVHGSLGDTSHSEDTLPKANTDSLEPAG

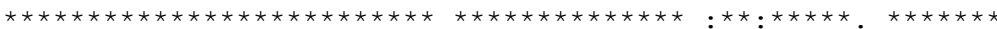

PSSPASVTVTVGDEGADTPVGATPLIGDESENLEGDGDLRGGRILLGHATKSFPSSPSKG PSSPASVTVTVGDEGADTPVGATPLIGDEPENLEGD----GGRILLGHATKSFPSSPSKG

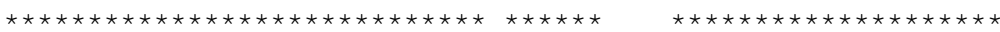

GSCPSRAKMSMTGAGKSPPSVQSLAMRLLSMPGAQGAAAAGSEPPPATTSPEGQPKVHRA GACPSRAKMSMTGAGKSPPSVQSLAMRLLSMPGAQGTAAAGPEPPPATTSPEGQPKVHRA

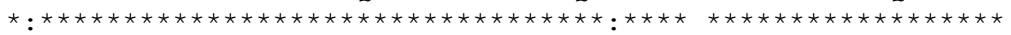

RKTMSKPGNGQP PVPEKRPPEIQHFRMSDDVHSLGKVTSDLAKRRKLNSGGGLSEELGSA 300 RKTMSKPGNGQPPVPEKRPPEVQHFRMSDDVHSLGKVTSDVAKRRKLNSGNGLSEELGSA

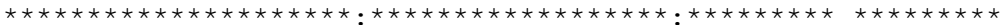

RRSGEVTLTKGDPGSLEEWETVVGDDFSLYYDSYSVDERVDSDSKSEVEALTEQLSEEEE RGSGEVTLEKGNPRSLEEWETVVGDDFSLYYDSYSVDERVDSDSKSEVEALAEQLSEEEE

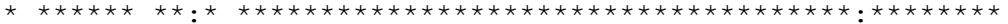

EEEEEEEEE-EEEEEEEEEEEDEESGNOSDRSGSSGRRKAKKKWRKDSPWVKPSRKRRKR EEEEEEEEEEEEEEEEEEEEEDEESGNQSDRSGSSGRRKAKKKWRKDS PWVKPTRKRRKR EPARAKEPRGVNGVGSSGPSEYMEVPLGSLELPSEGTLSPNHAGVSNDTSSLETERGFEE $\star * \star \star * \star * \star * \star *$

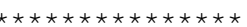

LPLCSCRMEAPKIDRISERAGHKCMATESVDGELSGCNAA I LKRETMRPSSRVALMVLCE LPLCSCRMEAPKI DRI SERAGHKCMATES I DGELSGCNAA I LKRETMRPSSRVALMVLCE

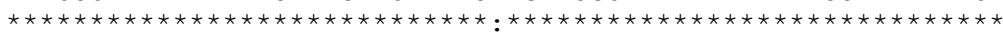

THRARMVKHHCCPGCGYFCTAGTFLECHPDFRVAHRFHKACVSQLNGMVFCPHCGEDASE THRARMVKHHCCPGCGYFCTAGTFLECHPDFRVAHRFHKACVSQLNGMVFCPHCGEDASE

AQEVTI PRGDGVTPPAGTAAPAPPPLSQDVPGRADTSQPSARMRGHGEPRRPPCDPLADT AQEVTIPRGDGGTPPAGTAAPAPPPLAQDAPGRADTSQPSARMRGHGEPRRPPCDPLADT

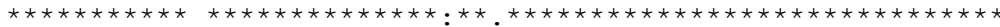

IDSSGPSLTLPNGGCLSAVGLPLGPGREALEKALVIQESERRKKLRFHPRQLYLSVKQGE IDSSGPSLTLPNGGCLSAVGLPPGPGREALEKALVIQESERRKKLRFHPRQLYLSVKQGE

LQKVILMLLDNLDPNFQSDQQSKRTPLHAAAQKGSVEICHVLLQAGANINAVDKQQRTPL LQKVILMLLDNLDPNFQSDQQSKRTPLHAAAQKGSVEICHVLLQAGANINAVDKQQRTPL MEAVVNNHLEVARYMVQRGGCVYSKEEDGSTCLHHAAKI GNLEMVSLLLSTGQVDVNAQD

SGGWTPI IWAAEHKH IEVIRMLLTRGADVTLTDNEENICLHWASFTGSAAIAEVLLNARC SGGWTP I IWAAEHKHIDVIRMLLTRGADVTLTDNEENICLHWASFTGSAAIAEVLLNARC

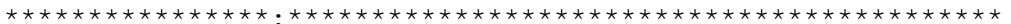
DLHAVNYHGDTPLHIAARESYHDCVLLFLSRGANPELRNKEGDTAWDLTPERSDVWFALQ LNRKLRLGVGNRAIRTEKI ICRDVARGYENVP I PCVNGVDGE PCPEDYKY I SENCETSTM 1008 
[Human]-G9a

[13LGS]-G9a

[Human]-G9a

[13LGS]-G9a

[Human]-G9a

[13LGS]-G9a

[Human]-G9a

[13LGS]-G9a

[Human] -G9a

[13LGS ]-G9a
NIDRNITHLQHCTCVDDCSSSNCLCGQLSIRCWYDKDGRLLQEFNKIEPPLIFECNQACS NIDRNITHLQHCTCVDDCSSSNCLCGQLS IRCWYDKDGRLLQEFNKIE P PLIFECNQACS

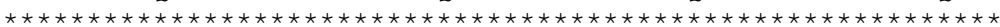

CWRNCKNRVVQSGIKVRLQLYRTAKMGWGVRALQT I PQGTF ICEYVGELISDAEADVRED CWRNCKNRVVQSGIKVRLQLYRTAKMGWGVRALQT I PQGTF ICEYVGELISDAEADVRED

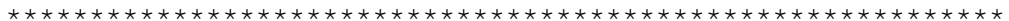

DSYLFDLDNKDGEVYCI DARYYGNISRF INHLCDPNI I PVRVFMLHQDLRFPRIAFFSSR DSYLFDLDNKDGEVYCIDARYYGNISRF INHLCDPNI I PVRVFMLHQDLRFPRIAFFSSR

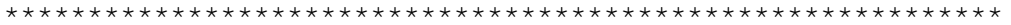

DIRTGEELGFDYGDRFWDIKSKYFTCQCGSEKCKHSAEAIALEQSRLARLDPHPELLPEL DIRTGEELGFDYGDRFWDIKSKYFTCQCGSEKCKHSAEAIALEQSRLARLDPHPELLPEL

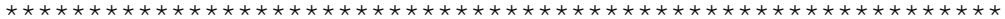

GSLPPVNT $\quad 1233$

GSLPPVNS $\quad 1256$

$\star \star \star \star \star \star \star *:$
1045

1068

1105

1128

1165

1188

1248

\# Percent Identity Matrix - created by Clustal2.1

\#
1: [Human]-G9a $100.00 \quad 97.62$
2: [13LGS]-G9a $97.62 \quad 100.00$ 


\section{Histone $\mathrm{H} 3$}

CLUSTAL O(1.2.1) multiple sequence alignment

[Human]-H3 MARTKQTARKSTGGKAPRKQLATKVARKSAPATGGVKKPHRYRPGTVALREIRRYQKSTE

[Human] $-\mathrm{H} 3$

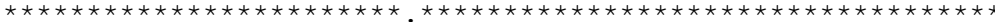

[13LGS $]-\mathrm{H} 3$

LLIRKLPFQRLMREIAQDFKTDLRFQSSAVMALQEACESYLVGLFEDTNLCVIHAKRVTI LLIRKLPFQRLVREIAQDFKTDLRFQSSAVMALQEASEAYLVGLFEDTNLCAIHAKRVTI

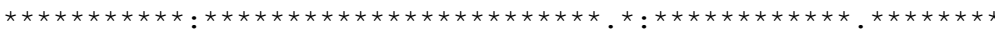

[Human ] $-\mathrm{H} 3$

MPKDIQLARRIRGERA

136

[13LGS ] $-\mathrm{H} 3$

MPKDIQLARRIRGERA

136

\# Percent Identity Matrix - created by Clustal2.1

\#
1: [Human] $-\mathrm{H} 3$
100.00
96.32
2: [13LGS]-H3
96.32100 .00 


\section{HSP90-alpha}

CLUSTAL O(1.2.1) multiple sequence alignment

[Human ]-HSP90

[13LGS]-HSP9O

[Human]-HSP90

[13LGS]-HSP 90

[Human]-HSP 90

[13LGS]-HSP90

[Human]-HSP90

[13LGS]-HSP 90

[Human]-HSP 90

[13LGS]-HSP90

[Human]-HSP90

[13LGS]-HSP 90

[Human] -HSP 90

[13LGS]-HSP 90

[Human]-HSP 90

[13LGS]-HSP 90

[Human ]-HSP90

[13LGS ]-HSP 90

[Human]-HSP90

[13LGS]-HSP90

[Human]-HSP 90

[13LGS]-HSP 90

[Human]-HSP90

[13LGS]-HSP90

[Human]-HSP90

[13LGS]-HSP90
MPEETOTODOPMEEEEVETFAFOAEIAOLMSLI INTFYSNKEIFLRELISNSSDALDKIR MPEETQTQDQPMEEEEVETFAFQAEIAQLMSLIINTFYSNKEIFLRELISNSSDALDKIR

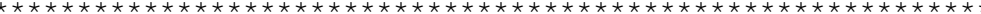

YESLTDPSKLDSGKELH INLI PNKQDRTLTIVDTGIGMTKADL INNLGTIAKSGTKAFME YESLTDPSKLDSGKELH INLI PNKQDRTLTIVDTGIGMTKADL INNLGTIAKSGTKAFME

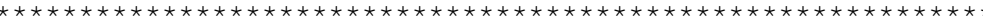

ALQAGADISMIGQFGVGFYSAYLVAEKVTVITKHNDDEQYAWESSAGGSFTVRTDTGEPM ALQAGADISMIGQFGVGFYSAYLVAEKVTVITKHNDDEQYAWESSAGGSFTVRTDTGEPM

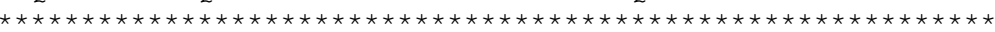

GRGTKVILHLKEDQTEYLEERRIKEIVKKHSQFIGYPITLFVEKERDKEVSDDEAEEKED GRGTKVI LHLKEDQTEYLEERRIKEIVKKHSQFIGYPITLFVEKERDKEVSDDEAEEKED

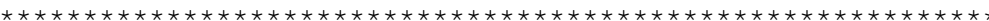

KEEEKEKEEKESEDKPEIEDVGSDEEE-EKKDGDKKKKKKIKEKY IDQEELNKTKPIWTR KEEEKEKEEKEPDDKPEIEDVGSDEEEEEKKDGDKKKKKKIKEKY IDQEELNKTKPIWTR

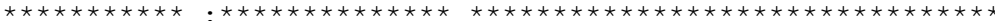

NPDDITNEEYGEFYKSLTNDWEDHLAVKHFSVEGQLEFRALLFVPRRAPFDLFENRKKKN NPDDITNEEYGEFYKSLTNDWEDHLAVKHFSVEGQLEFRALLFVPRRAPFDLFENRKKKN

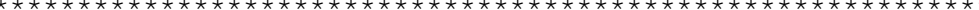

NIKLYVRRVF IMDNCEELI PEYLNF IRGVVDSEDL PLNISREMLQQSKILKVIRKNLVKK NI KLYVRRVF IMDNCEELI PEYLNF IRGVVDSEDLPLNI SREMLQQSKILKVIRKNLVKK

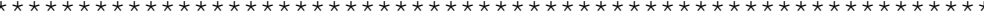

CLELFTELAEDKENYKKFYEOFSKNIKLGIHEDSONRKKLSELLRYYTSASGDEMVSLKD CLELFTELAEDKENYKKFYEQFSKNIKLGIHEDSONRKKLSELLRYYTSASGDEMVSLKD

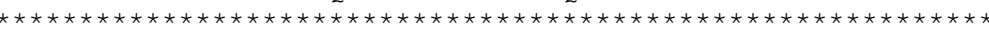

YCTRMKENQKH IYYITGETKDQVANSAFVERLRKHGLEVI YMIEP I DEYCVQQLKEFEGK YCTRMKENQKHIYYITGETKDQVANSAFVERLRKHGLEVIYMIEPIDEYCVOQLKEFEGK

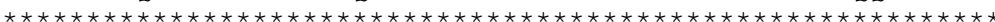

TLVSVTKEGLELPEDEEEKKKQEEKKTKFENLCKIMKDILEKKVEKVVVSNRLVTSPCCI TLVSVTKEGLELPEDEEEKKKQEEKKTKFENLCKIMKDILEKKVEKVVVSNRLVTSPCCI

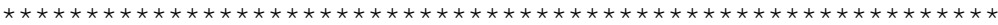

VTSTYGWTANMERIMKAQALRDNSTMGYMAAKKHLEINPDHS I IETLRQKAEADKNDKSV VTSTYGWTANMERIMKAQALRDNSTMGYMAAKKHLEINPDHS I IETLRQKAEADKNDKSV

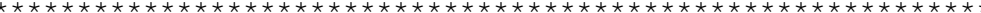

KDLVILLYETALLSSGFSLEDPQTHANRIYRMIKLGLGIDEDDPTADDTSAAVTEEMPPI KDLVILLYETALLSSGFSLEDPQTHANRIYRMIKLGLGIDEDDPTADDTSAAVTEEMPPL

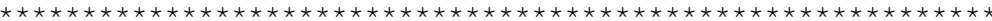

\# Percent Identity Matrix - created by Clustal2.1

$\begin{array}{ll}\text { EGDDDTSRMEEVD } & 732 \\ \text { EGDDDTSRMEEVD } & 733\end{array}$
西 
p53

CLUSTAL O(1.2.1) multiple sequence alignment

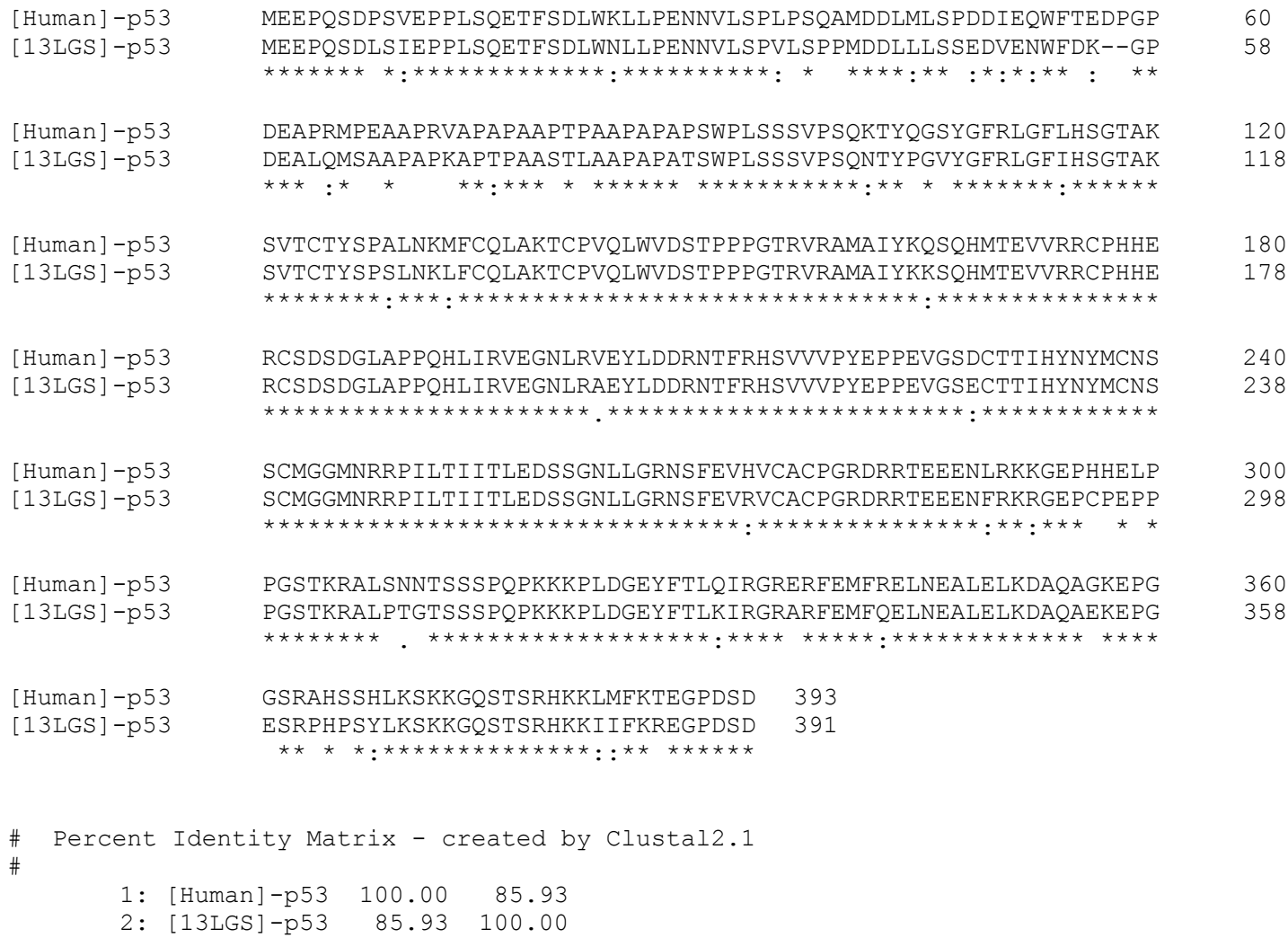




\section{Appendix B: Antibodies used for Western blotting}

Table B1:

Supplier and catalog number of antibodies used in Western blotting

\begin{tabular}{lcr}
\hline Protein Target & Supplier & Catalog \# \\
\hline SUV39H1 & Cell Signaling Technology & 8729 \\
SMYD2 & Cell Signaling Technology & 9734 \\
SET8 & Cell Signaling Technology & 2996 \\
SET7/9 & Cell Signaling Technology & 2813 \\
G9a & Cell Signaling Technology & 3306 \\
ASH2L & Cell Signaling Technology & 5019 \\
RBBP5 & Cell Signaling Technology \\
H3K4me & Abcam & 13171 \\
H3K9me & Abcam & ab8895 \\
H3K27me & Active Motif & ab8898 \\
H3K36me & Active Motif & 39890 \\
HSP90 & Genetex & 39892 \\
pRb & Cell Signaling Technology & GTX109753 \\
pan-methyllysine & Stressmarq & 9309 \\
p53-K370me & Ameritech Biomedicines & SPC-158F \\
p53-K372me & Genetex & ATB-H0007 \\
\hline
\end{tabular}




\section{Appendix C: Validation Tests for Commercial Activity Assays}

Commercial activity assays used in this research were subjected to rigorous quality control and validation steps by the manufacturer. Three versions of the Epigentek (Farmingdale, NY) assay kits - EpiQuik Histone Methyltransferase Activity/Inhibition Assay Kit - were used, each specific for one of three histone H3 lysine residues, K4 (Cat\#: P-3002), K9 (Cat\#: P-3003) or K27 (Cat\#: P-3005). In order to confirm the validity of the obtained results for 13LGS tissue, additional tests were completed as described herein.

\section{Determination of Protein Load}

A protein dilution curve was completed in order to find the amount of protein required to produce appreciable levels of signal, while not oversaturating increases in methyltransferase activity as a result of overloading. Results and chosen protein dilutions may be found in Table C1. In the case of skeletal muscle samples, protein load amounts exceeded those set out by the manufacturer (4-30 $\mu \mathrm{g}$ ) and as such, a decreased level of assay buffer was used to keep well volumes consistent between assays. Protein dilution curves demonstrated that protein amounts of up to $35 \mu \mathrm{g}$ in liver, and up to $100 \mu \mathrm{g}$ in skeletal muscle, did not produce signal saturation and were appropriate for the final load amounts chosen for the assays $(30 \mu \mathrm{g}$ in liver, and $70 \mu \mathrm{g}$ in skeletal muscle tissue).

\begin{tabular}{|c|c|c|c|c|c|c|}
\hline \multirow[b]{2}{*}{ Tissue } & \multicolumn{2}{|c|}{ H3K4 } & \multicolumn{2}{|c|}{ H3K9 } & \multicolumn{2}{|c|}{ H3K27 } \\
\hline & $\begin{array}{c}\text { Protein Amount } \\
\text { (ug) }\end{array}$ & $\mathrm{OD}_{450}$ & $\begin{array}{c}\text { Protein Amount } \\
\text { (ug) }\end{array}$ & $\mathrm{OD}_{450}$ & $\begin{array}{c}\text { Protein Amount } \\
\text { (ug) }\end{array}$ & $\mathrm{OD}_{450}$ \\
\hline MUS & 45 & 0.448 & 70 & 0.432 & 70 & 0.299 \\
\hline LIV & 30 & 0.443 & 30 & 0.369 & 30 & 0.370 \\
\hline
\end{tabular}

\section{Signal within Effective Range of Assay}

A standard curve was completed in order to find the effective working range of $\mathrm{OD}_{450}$ (Figure C1). The absorbance of each assay well was determined to be well within the effective range produced by no-protein blank wells and purified enzyme positive-control wells $\left(\mathrm{OD}_{450}\right.$ between approximately 0.1 and 3.0 ), further ensuring the validity of the results.

\section{Calculation of Methyltransferase Activity}

In order to calculate KMT activity at each methylation target site, OD values were manipulated using a manufacturer-provided formula and the $\Delta \mathrm{OD} / \mathrm{ng}$ (from the standard curve),

$$
\operatorname{Activity~}(\mathrm{ng} / \mathrm{h} / \mathrm{mg})=\frac{\text { OD }(\text { sample }- \text { blank })}{\text { Protein Amount }(\mu g) \times \text { hour } \times \Delta \mathrm{OD} / \mathrm{ng}} \times 1000 .
$$


A
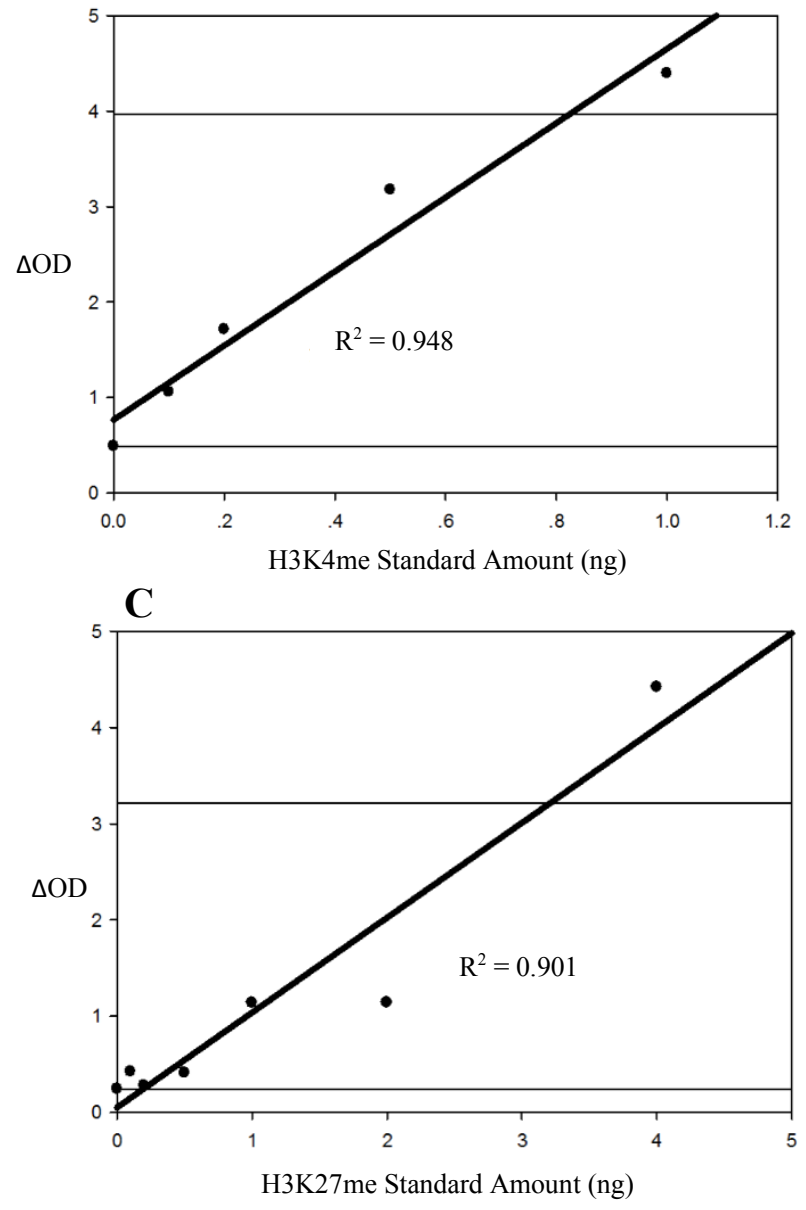

B

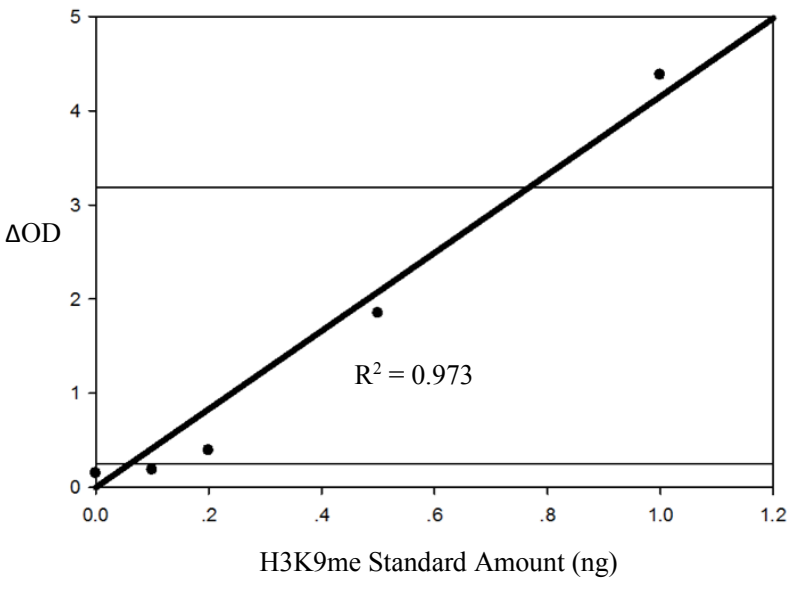

Figure C1: The EpiQuik histone methyltransferase activity/inhibition assay kit was used to generate a standard curve using known dilutions of specific methylated histone substrates that are detected by an antibody specific for A) methylated H3K4 assayed from 0-1.0 ng, B) methylated $\mathrm{H} 3 \mathrm{~K} 9$ assayed from 0-1.0 ng, and C) methylated H3K27 assayed from 0-4.0 ng. The enzymatic reaction was incubated at $37^{\circ} \mathrm{C}$ for one and a half hours, followed by capture antibody and detection antibody incubations. Colorimetric intensity was measured at an absorbance of $450 \mathrm{~nm}$. Results were plotted as a linear regression along with R-squared measurements and equations for the linear regression line. Minimal blank values and purified-KMT enzyme positive-control values are displayed as a lower and upper reference line, respectively. 


\section{Appendix D: Conditions used for Western blotting}

Table D1:

Antibodies and specific conditions for Western blot experiments

\begin{tabular}{|c|c|c|c|c|c|c|c|c|}
\hline$\frac{1^{0} \text { Antibody }}{\underline{\text { Target }}}$ & Tissue & $\begin{array}{c}\underline{\text { Loaded }} \\
\underline{\text { Protein }} \\
\underline{\text { Amount }(\mu g)} \\
\end{array}$ & Gel Type & $\frac{\text { Run }}{\underline{\text { Time }}}$ & $\frac{\text { Transfer }}{\text { Time (min) }}$ & $\frac{\text { Membrane }}{\underline{\text { Blocking }}}$ & $\frac{1^{0} \text { Antibody }}{\text { Incubation }}$ & $\frac{2^{0} \text { Antibody }}{\underline{\text { Incubation }}}$ \\
\hline SUV39H1 & MUS & 20 & $15 \%$ tris-glycine & $240 ; 180$ & $90 ; 160 \mathrm{~mA}$ & $\begin{array}{c}3.5 \% \text { milk; } \\
15 \text { min }\end{array}$ & $\begin{array}{c}1: 1000 ; \\
\text { over } 2 \text { nights }\end{array}$ & $\begin{array}{l}1: 8000 ; \\
20 \mathrm{~min}\end{array}$ \\
\hline \multirow{2}{*}{ SMYD2 } & MUS & 20 & $15 \%$ tris-glycine & $90 ; 180$ & $90 ; 160 \mathrm{~mA}$ & $\begin{array}{c}2.5 \% \text { milk; } \\
30 \mathrm{~min}\end{array}$ & $\begin{array}{c}\text { 1:1000; } \\
\text { overnight }\end{array}$ & $\begin{array}{l}1: 6000 \\
20 \mathrm{~min}\end{array}$ \\
\hline & LIV & 20 & $12 \%$ tris-glycine & $90 ; 180$ & $90 ; 160 \mathrm{~mA}$ & $\begin{array}{l}10 \% \text { milk; } \\
30 \text { min }\end{array}$ & $\begin{array}{c}\text { 1:1000; } \\
\text { overnight }\end{array}$ & $\begin{array}{l}1: 8000 \\
15 \mathrm{~min}\end{array}$ \\
\hline \multirow{2}{*}{ SET8 } & MUS & 20 & $15 \%$ tris-glycine & $90 ; 180$ & $90 ; 160 \mathrm{~mA}$ & $\begin{array}{l}2.5 \% \text { milk; } \\
30 \mathrm{~min}\end{array}$ & $\begin{array}{c}\text { 1:1000; } \\
\text { overnight }\end{array}$ & $\begin{array}{l}1: 6000 ; \\
20 \text { min }\end{array}$ \\
\hline & LIV & 20 & $10 \%$ tris-glycine & $60 ; 180$ & $90 ; 160 \mathrm{~mA}$ & $\begin{array}{l}2.5 \% \text { milk; } \\
30 \mathrm{~min}\end{array}$ & $\begin{array}{c}\text { 1:1000; } \\
\text { overnight }\end{array}$ & $\begin{array}{l}1: 6000 ; \\
20 \mathrm{~min}\end{array}$ \\
\hline \multirow{2}{*}{ SET7/9 } & MUS & 25 & $12 \%$ tris-glycine & $75 ; 180$ & $90 ; 160 \mathrm{~mA}$ & $\begin{array}{c}1 \% \text { milk; } \\
10 \text { min }\end{array}$ & $\begin{array}{c}1: 1000 ; \\
\text { over } 2 \text { nights }\end{array}$ & $\begin{array}{l}1: 6000 ; \\
20 \text { min }\end{array}$ \\
\hline & LIV & 20 & $12 \%$ tris-glycine & $75 ; 180$ & $90 ; 160 \mathrm{~mA}$ & $\begin{array}{c}1 \% \text { milk; } \\
10 \mathrm{~min}\end{array}$ & $\begin{array}{c}\text { 1:1000; } \\
\text { overnight }\end{array}$ & $\begin{array}{l}1: 6000 \\
20 \mathrm{~min}\end{array}$ \\
\hline
\end{tabular}


Table D1:

Antibodies and specific conditions for Western blot experiments

\begin{tabular}{|c|c|c|c|c|c|c|c|c|}
\hline$\frac{\mathbf{1}^{0} \text { Antibody }}{\underline{\text { Target }}}$ & $\underline{\text { Tissue }}$ & $\begin{array}{c}\underline{\text { Loaded }} \\
\underline{\text { Protein }} \\
\underline{\text { mount }(\mu g)} \\
\end{array}$ & Gel Type & $\frac{\text { Run }}{\underline{\text { Time }}}$ & $\begin{array}{l}\text { Transfer } \\
\text { Time (min) }\end{array}$ & $\frac{\text { Membrane }}{\text { Blocking }}$ & $\frac{1^{0} \text { Antibody }}{\text { Incubation }}$ & $\frac{2^{\circ} \text { Antibody }}{\text { Incubation }}$ \\
\hline \multirow{2}{*}{ G9a } & MUS & 40 & $6 \%$ tris-glycine & $90 ; 180$ & $180 ; 160 \mathrm{~mA}$ & $\begin{array}{l}1 \% \text { milk; } \\
20 \text { min }\end{array}$ & $\begin{array}{c}\text { 1:1000; } \\
\text { overnight }\end{array}$ & $\begin{array}{l}1: 8000 \\
40 \mathrm{~min}\end{array}$ \\
\hline & LIV & 30 & $6 \%$ tris-glycine & $50 ; 180$ & $180 ; 160 \mathrm{~mA}$ & $\begin{array}{l}1 \% \text { milk; } \\
10 \text { min }\end{array}$ & $\begin{array}{c}\text { 1:1000; } \\
\text { over } 2 \text { nights }\end{array}$ & $\begin{array}{l}1: 6000 \\
20 \mathrm{~min}\end{array}$ \\
\hline \multirow{2}{*}{ ASH2L } & MUS & 25 & $12 \%$ tris-glycine & $180 ; 180$ & $100 ; 160 \mathrm{~mA}$ & $\begin{array}{l}1 \% \text { milk; } \\
30 \text { min }\end{array}$ & $\begin{array}{c}\text { 1:1000; } \\
\text { overnight }\end{array}$ & $\begin{array}{l}1: 8000 \\
20 \mathrm{~min}\end{array}$ \\
\hline & LIV & 20 & $12 \%$ tris-glycine & $160 ; 180$ & $100 ; 160 \mathrm{~mA}$ & $\begin{array}{l}2.5 \% \text { milk; } \\
20 \mathrm{~min}\end{array}$ & $\begin{array}{c}\text { 1:1000; } \\
\text { over } 2 \text { nights }\end{array}$ & $\begin{array}{c}1: 10000 \\
15 \mathrm{~min}\end{array}$ \\
\hline \multirow{2}{*}{ RBBP5 } & MUS & 25 & $12 \%$ tris-glycine & $180 ; 180$ & $100 ; 160 \mathrm{~mA}$ & $\begin{array}{l}5 \% \text { milk; } \\
20 \text { min }\end{array}$ & $\begin{array}{c}\text { 1:1000; } \\
\text { overnight }\end{array}$ & $\begin{array}{l}1: 8000 \\
20 \mathrm{~min}\end{array}$ \\
\hline & LIV & 35 & $12 \%$ tris-glycine & $180 ; 180$ & $100 ; 160 \mathrm{~mA}$ & $\begin{array}{c}5 \% \text { milk; } \\
20 \text { min }\end{array}$ & $\begin{array}{c}\text { 1:1000; } \\
\text { over } 2 \text { nights }\end{array}$ & $\begin{array}{l}1: 8000 \\
20 \mathrm{~min}\end{array}$ \\
\hline \multirow{2}{*}{$\mathrm{H} 3 \mathrm{~K} 4 \mathrm{me}$} & MUS & 35 & $15 \%$ tris-tricine & $\begin{array}{l}30 ; 60+ \\
130 ; 160\end{array}$ & $120 ; 30 \mathrm{~V}$ & $\begin{array}{l}4 \% \text { milk; } \\
20 \text { min }\end{array}$ & $\begin{array}{c}\text { 1:1000; } \\
\text { over } 2 \text { nights }\end{array}$ & $\begin{array}{l}1: 6000 \\
25 \mathrm{~min}\end{array}$ \\
\hline & LIV & 35 & $15 \%$ tris-tricine & $\begin{array}{l}25 ; 60+ \\
140 ; 160\end{array}$ & $120 ; 30 \mathrm{~V}$ & $\begin{array}{l}1 \% \text { milk; } \\
30 \text { min }\end{array}$ & $\begin{array}{c}\text { 1:1000; } \\
\text { overnight }\end{array}$ & $\begin{array}{l}1: 8000 \\
20 \mathrm{~min}\end{array}$ \\
\hline \multirow{2}{*}{$\mathrm{H} 3 \mathrm{~K} 9 \mathrm{me}^{3}$} & MUS & 35 & $15 \%$ tris-tricine & $\begin{array}{l}20 ; 60+ \\
130 ; 160\end{array}$ & $120 ; 30 \mathrm{~V}$ & $\begin{array}{l}6 \% \text { milk; } \\
25 \text { min }\end{array}$ & $\begin{array}{c}\text { 1:1000; } \\
\text { overnight }\end{array}$ & $\begin{array}{l}1: 6000 \\
20 \mathrm{~min}\end{array}$ \\
\hline & LIV & 35 & $15 \%$ tris-tricine & $\begin{array}{l}25 ; 60+ \\
140 ; 160\end{array}$ & $120 ; 30 \mathrm{~V}$ & $\begin{array}{l}0.5 \% \text { milk; } \\
5 \text { min }\end{array}$ & $\begin{array}{c}\text { 1:1000; } \\
\text { overnight }\end{array}$ & $\begin{array}{l}1: 8000 \\
30 \mathrm{~min}\end{array}$ \\
\hline
\end{tabular}


Table D1:

Antibodies and specific conditions for Western blot experiments

\begin{tabular}{|c|c|c|c|c|c|c|c|c|}
\hline$\frac{\mathbf{1}^{0} \text { Antibody }}{\underline{\text { Target }}}$ & $\underline{\text { Tissue }}$ & $\begin{array}{c}\underline{\text { Loaded }} \\
\underline{\text { Protein }} \\
\underline{\text { mount }(\mu g)} \\
\end{array}$ & Gel Type & $\frac{\text { Run }}{\underline{\text { Time }}}$ & $\begin{array}{l}\text { Transfer } \\
\text { Time (min) }\end{array}$ & $\frac{\text { Membrane }}{\underline{\text { Blocking }}}$ & $\frac{1^{0} \text { Antibody }}{\underline{\text { Incubation }}}$ & $\begin{array}{l}\frac{2^{0} \text { Antibody }}{\text { Incubation }} \\
\underline{\underline{y}}\end{array}$ \\
\hline H3K27me & MUS & 40 & $15 \%$ tris-tricine & $\begin{array}{l}35 ; 60+ \\
145 ; 160\end{array}$ & $120 ; 30 \mathrm{~V}$ & $\begin{array}{c}2 \% \text { milk; } \\
10 \text { min }\end{array}$ & $\begin{array}{c}\text { 1:1000; } \\
\text { over } 2 \text { nights }\end{array}$ & $\begin{array}{l}1: 6000 \\
40 \mathrm{~min}\end{array}$ \\
\hline \multirow{2}{*}{$\mathrm{H} 3 \mathrm{~K} 36 \mathrm{me}^{2}$} & MUS & 35 & $15 \%$ tris-tricine & $\begin{array}{l}25 ; 60+ \\
140 ; 160\end{array}$ & $120 ; 30 \mathrm{~V}$ & $\begin{array}{l}4 \% \text { milk; } \\
20 \text { min }\end{array}$ & $\begin{array}{c}\text { 1:1000; } \\
\text { over } 2 \text { nights }\end{array}$ & $\begin{array}{l}1: 6000 \\
25 \mathrm{~min}\end{array}$ \\
\hline & LIV & 50 & $15 \%$ tris-tricine & $\begin{array}{l}25 ; 60+ \\
140 ; 160\end{array}$ & $120 ; 30 \mathrm{~V}$ & $\begin{array}{l}5 \% \text { milk; } \\
5 \text { min }\end{array}$ & $\begin{array}{c}1: 1000 ; \\
\text { over } 2 \text { nights }\end{array}$ & $\begin{array}{l}1: 6000 \\
40 \mathrm{~min}\end{array}$ \\
\hline HSP90 & MUS & 20 & $8 \%$ tris-glycine & $90 ; 180$ & $100 ; 160 \mathrm{~mA}$ & $\begin{array}{l}2.5 \% \text { milk; } \\
20 \mathrm{~min}\end{array}$ & $\begin{array}{c}\text { 1:1000; } \\
\text { overnight }\end{array}$ & $\begin{array}{l}1: 6000 \\
20 \mathrm{~min}\end{array}$ \\
\hline $\begin{array}{c}\text { pan- } \\
\text { methyllysine }\end{array}$ & MUS & 20 & $8 \%$ tris-glycine & $90 ; 180$ & $100 ; 160 \mathrm{~mA}$ & $\begin{array}{c}8 \% \text { milk; } \\
30 \text { min }\end{array}$ & $\begin{array}{c}\text { 1:1000; } \\
\text { overnight }\end{array}$ & $\begin{array}{l}1: 6000 \\
25 \mathrm{~min}\end{array}$ \\
\hline $\mathrm{p} 53-\mathrm{K} 370 \mathrm{me}^{2}$ & LIV & 30 & $15 \%$ tris-glycine & $200 ; 180$ & $90 ; 160 \mathrm{~mA}$ & $\begin{array}{l}9 \% \text { milk; } \\
30 \text { min }\end{array}$ & $\begin{array}{c}\text { 1:1000; } \\
\text { overnight }\end{array}$ & $\begin{array}{l}1: 8000 \\
40 \mathrm{~min}\end{array}$ \\
\hline p53-K372me & LIV & 15 & $15 \%$ tris-glycine & $200 ; 180$ & $90 ; 160 \mathrm{~mA}$ & $\begin{array}{l}7 \% \text { milk; } \\
45 \text { min }\end{array}$ & $\begin{array}{c}\text { 1:1000; } \\
\text { overnight }\end{array}$ & $\begin{array}{l}1: 8000 \\
20 \mathrm{~min}\end{array}$ \\
\hline
\end{tabular}




\section{Appendix E: Typical Western Blot Images}

Western blot images were scrutinized for flaws that may indicate sample or technical failures. A typical quantifiable Western blot image as was used for data analysis is shown below in Figure 1. For comparison, a blot that produced technical glitches interfering with several samples, requiring a further experiment to correct, is shown in Figure 2. Further details of the measures taken to correct sample or experimental failures are available in the Western blotting section within the Materials and Methods.

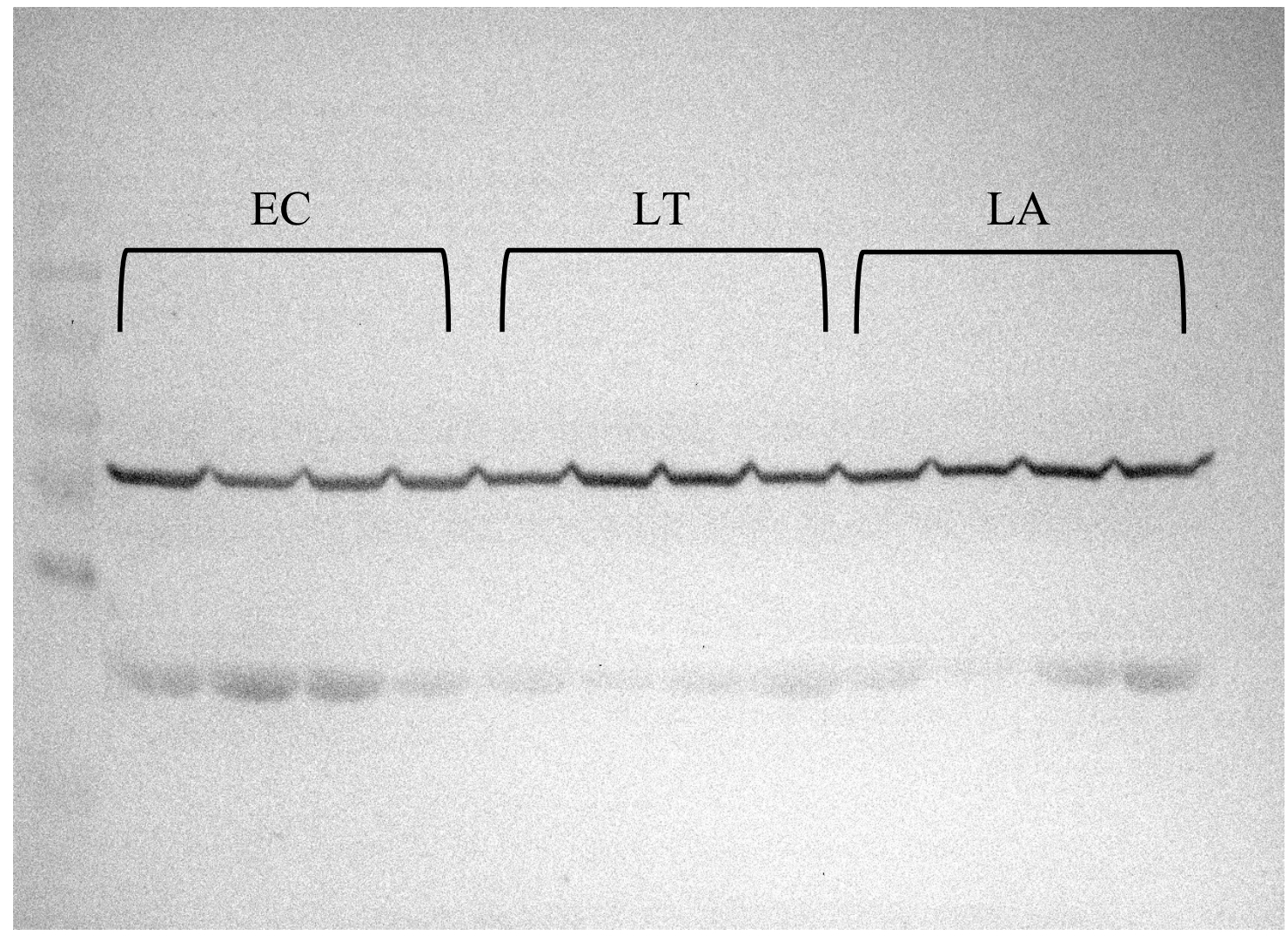

Figure 1: Typical Western blot image produced using anti-SET8 antibody and muscle samples from the indicated time points 


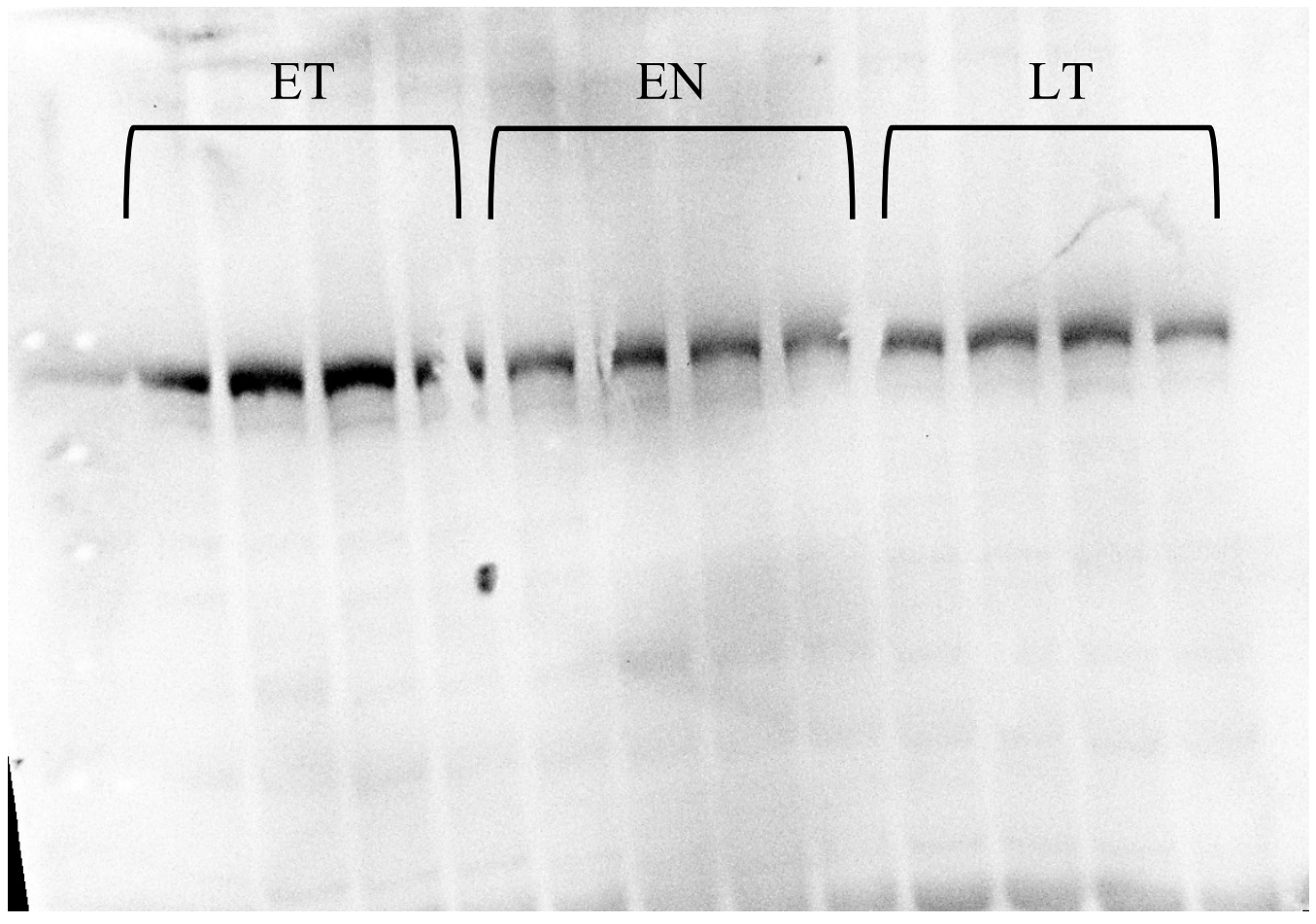

Figure 2: Typical Western blot image produced using anti-ASH2L antibody and muscle samples from the indicated time points. Technical glitches required the re-running of the EN and ET time-points. 